\title{
ALTERNATING CURRENT DISTRIBUTION IN CYLINDRICAL CONDUCTORS
}

\author{
By Chester Snow
}

\section{ABSTRACT}

The fundamental equations of Maxwell for the scalar and vector potentials $\phi$ and $A$ are simplified by an approximation which is valid from the lowest to the highest (radio) frequencies. This approximation is due to the difference in the order of magnitude of the electrical conductivity of dielectrics and conductors. The case of $N$ conducting cylindrical groups surrounded by any number of dielectrics is discussed and forms of solution obtained for $\phi$ and $A$ which are proportional to $e^{i p t-\gamma z}$ representing a simple possible type of propagation along the cylinders. These lead to general formulas for the coefficients of leakage, capacity, resistance, and inductance (all of which are functions of the frequency in the general case) as well as expressions for the attenuation and phase velocity. The mean energy relations are also expressed in terms of these coefficients. Application is made to the case of two circular cylinders of different conductivity, permeability, and radius, surrounded by a homogeneous, slightly conductive dielectric. Asymptotic formulas for the alternating current resistance $R$ and inductance $L$ of the line at high frequencies are obtained, which, together with the exact expressions for the coefficients of leakage and capacity, lead to highfrequency expressions for the attenuation and phase velocity. These are all functions of the frequency due to the fact that the current distribution is not uniform in the conductors.

\section{CONTENTS}

I. Introduction-Statement of problem

II. Fundamental equations.

1. Type of waves - The propagation constant $\gamma$

2. General approximations__._. 279

3. Differential equations and boundary conductions for $\phi$ and $A_{-} \quad 281$

III. The complex scalar potential $\phi \ldots$

1. Uniqueness of a solution. 284

2. Existence and uniqueness of a generalized Green's function $G \ldots \ldots$

3. The existence of $\phi$ and its integral representation

4. Properties and physical interpretation of the partial potentials $\phi_{\mathrm{n}}-\ldots$

5. The complex coefficients of leakage and capacity

IV. The complex vector potential $A$

1. Existence and properties of a magnetic flux function $M_{-}$

2. Integral equations for $A$ and $E$

3. Existence and uniqueness of a solution of (36)

4. Other methods of solving the integral equation (36)

(a) By normal functions......

(b) Method of successive integration at low frequencies

(c) Method of harmonic analysis.

(d) Mixed method

5. Form and properties of the solution 305

6. Coefficients of resistance and inductance 311 
V. The propagation constant $\gamma$. Attenuation and phase velocity _...- $\quad 313$

1. Determinantal equation for $\gamma$

2. Determination of constants to fit terminal conditions.....- 316

3. Special cases . .

(a) Case of two conductors

(b) Uniform proximity effect._................. 317

VI. Energy relations and mean values

VII. Examples and applications _..._.

1. Case of two conductors with circular symmetry _...... 321

2. Case of two circular, cylindrical conductors of different conductivity, permeability, and radius, surrounded by a homogeneous, slightly conducting dielectric. Mixed method.-

(a) First and second approximations at high frequency. Asymptotic formulas for $R, L$, and $\gamma$ with any

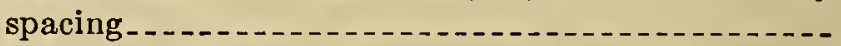

(b) Special case. Circular cylinder parallel to semi-infinite plane of finite conductivity

VIII. Summary

\section{INTRODUCTION-STATEMENT OF PROBLEM}

A knowledge of the mode of propagation and distribution of alternating current in a cylindrical system is important, for such currents are used in practice, and, moreover, the propagation of an arbitrary type of wave or "transient" may theoretically be found in terms of the periodic solution by the use of Fourier's integral or by complex integration. ${ }^{1}$ In working out such distinct problems as that of propagation along parallel wires where the dielectric extends to infinity, and along a cable systẹm where a conductor extends to infinity, a number of formal similarities become evident. It may be difficult to see just how general are these similarities if the initial formulation of the problem is not sufficiently general. Some of the approximations to be made are common to all problems of this type. Moreover, the connections between the field vectors and such physical concepts as resistance, inductance, and capacity may be made in a manner which is quite inclusive. In this paper a discussion of the general problem is undertaken in the hope that it may prove useful in outlining the procedure or forecasting the results in particular problems of this type.

\section{Ii. FUNDAMENTAL EQUATIONS}

\section{TYPE OF WAVES - THE PROPAGATION CONSTANT $\gamma$}

If all the conductors and dielectrics have their generating lines parallel to the $z$ axis, the electric vector may be represented by the real part of $E e^{\mathrm{ipt- \gamma ^{2 }}}$ and the magnetic induction by the real part of

1 Thornton C. Fry., Phys. Rev., p. 115; August, 1919. 
$B e^{\mathrm{ipt}-\gamma^{z}}$ where $p$ is $2 \pi$ times the frequency, and $\gamma$ is the complex propagation constant which may be written

$$
\gamma=b+\frac{i p}{V}
$$

The real quantities $b$ and $V$ represent the attenuation constant and phase velocity, respectively, of this type of wave. There will be a finite number of types of waves or values of $\gamma$ possible for a given system, and we shall first consider the case where only one type is present. It will be more convenient to deal with the complex scalar and vector potentials $\phi$ and $A$, respectively, and to derive $E$ and $B$ from them by the relations

$$
\left.\begin{array}{l}
E=-\nabla \phi-i p A \\
B=\operatorname{curl} A=\mu H
\end{array}\right\}
$$

The electromagnetic cgs system of units will be used throughout with the exception of the dielectric constant $k$, which will be taken in electrostatic cgs units. The electrical conductivity being $\lambda$, and the ratio of the units being $c=3 \times 10^{10}$, the Maxwell equations

$$
\left.\begin{array}{ll}
\operatorname{curl} B=4 \pi \mu\left(\lambda+\frac{i p k}{4 \pi c^{2}}\right) E & \text { Div } B=O \\
\operatorname{curl} E=-i p B & \text { Div } E=O
\end{array}\right\}
$$

require that

$$
\left.\begin{array}{l|l}
\nabla^{2} A_{\mathbf{x}}+h^{2} A_{\mathbf{x}} & =\frac{\partial \chi}{\partial x} \\
\nabla^{2} A_{\mathbf{y}}+h^{2} A_{\mathbf{y}} & =\frac{\partial \chi}{\partial y} \\
\nabla^{2} A_{\mathbf{z}}+h^{2} A_{\mathbf{z}}=\frac{\partial \chi}{\partial z}=-\gamma \chi & \text { Where } \nabla^{2} \equiv \frac{\partial^{2}}{\partial x^{2}}+\frac{\partial^{2}}{\partial y^{2}} \\
\Delta^{2} \phi+h^{2} \phi & =-\frac{\partial \chi}{\partial t}=-i p \chi
\end{array} \mid \begin{array}{l}
h^{2}=\gamma^{2}-4 \pi i p \mu\left(\lambda+\frac{i p k}{4 \pi c^{2}}\right) \\
\chi=\frac{\partial A_{\mathbf{x}}}{\partial x}+\frac{\partial A_{\mathbf{y}}}{\partial y}-\gamma A_{\mathbf{z}}+\frac{\gamma^{2}-h^{2} \phi}{i p}
\end{array}\right\}
$$

Conversely any pair $\phi$ and $A$ which satisfy these equations (4) and give continuity to the tangential components of $E$ and $H$ will give the correct field vectors.

\section{GENERAL APPROXIMATIONS}

It will be assumed that $\gamma$ is a small quantity of the order of $\frac{1}{c}$ (first order). In all materials $k$ is of the order of unity, and hence the complex conductivity $a$, defined by

$$
a \equiv \lambda+\frac{i p k}{4 \pi c^{2}}
$$

will be very approximately equal to $\lambda$ in conductors; that is, a finite magnitude (for copper $\lambda=0.0006$ ). 
In dielectrics $\lambda$ is of the same order, or even smaller than the term $\frac{i p k}{4 \pi c^{2}}$. Thus for gutta-percha $\lambda=10^{-24}$ and $k=4$ so that $\frac{p k}{4 \pi c^{3}}=\frac{2 f}{10^{21}}$. It is therefore evident that for a range of frequency from one cycle up to the highest $\lambda$ will not be greater than the second order term $\frac{p k}{4 \pi c^{2}}$ even if the dielectric is ten thousand times a better conductor than gutta-percha, and consequently the complex conductivity will be a second order infinitesimal in all dielectrics. For the sake of the most general results we shall assume that $\lambda$ may be of the same order as $\frac{p k}{4 \pi c^{2}}$ or $\gamma^{2}$. The magnetic permeability $\mu$ will have the value 1 in all dielectrics and in nonmagnetic metals.

The cause of the wave is assumed to be in certain electromotive forces applied to the terminals of the conductor at, say, $z=0$ and $z=l$, where the cylindrical conductors are connected by networks of known impedances. The simple type of wave here proposed could not exist, strictly speaking, if the cylinders were not infinitely long in both directions. However, we are here concerned with engineering rather than optical applications, so that we may assume that the cylinders are so long that the regions of space in the neighborhood of the terminals, where this simple type of wave is departed from, are negligible compared to the regions between the terminals where it is sufficiently correct. Therefore, we may ignore these terminal variations of field or we may consider the term "end impedances" sufficiently elastic to include them. We shall assume that there is nothing analogous to these applied electromotive forces in a magnetic sense; no applied "magnetomotive forces" tending to magnetize the cylinders in the $z$ direction. Hence, the component of magnetic field in this direction $H_{z}$ will be everywhere infinitesimal, since it could only be produced by the $x y$ components of current which will be infinitesimal in the dielectrics and also in the conductors since the normal component of current in conductors must be continuous with its value outside. Hence, $A_{x}$ and $A_{y}$ will be negligible. We may, therefore, understand $A$ to mean the $z$ component $A_{z}$ in all that follows, since the others are negligible.

The first approximation to the soluticn will, therefore, lead to

$$
\left.\begin{array}{ll}
E_{x}=-\frac{\partial \phi}{\partial x} & B_{\mathbf{x}}=\frac{\partial A}{\partial y} \\
E_{\eta}=-\frac{\partial \phi}{\partial y} & B_{\mathrm{y}}=-\frac{\partial A}{\partial x} \\
E_{z}=E=-\frac{\partial \phi}{\partial z}-i p A_{z}=\gamma \phi-i p A & B_{\mathrm{z}}=0
\end{array}\right\}
$$

which hold at all points in the $x y$ plane. 


\section{DIFFERENTIAL EQUATIONS AND BOUNDARY CONDUCTIONS FOR $\phi$ AND $A$}

The electric component $E_{\mathrm{z}}$ (which will be designated by $E$ from now on) is of finite order of magnitude everywhere and is continuous at all boundaries. Since $E=\gamma \phi-i p A$, it follows that $A$ and $\gamma \phi$ are also finite. If $\phi$ and $A$ are continuous at all boundaries, the continuity of $E$ will be assured. Since $E_{\mathrm{x}}$ and $E_{\mathrm{y}}$ are negligible in conductors, $\phi$ must have a constant value at all points in each conducting section. Let $\phi=c_{\mathrm{n}}$ at all points in the plane section $S_{\mathrm{n}}(z=$ constant) of the $n^{\text {th }}$ conductor or group of conductors in contact. Suppose there are $N$ such groups. The tangential component of the electric field in the $x y$ plane will be zero (and hence continuous) at all boundaries between a dielectric and conductor or between two conductors. It will also be continuous at boundaries between two different dielectrics if $\phi$ is continuous there. At such surfaces the conservation of electricity, or solenoidal property of the total electric current, requires that $a \frac{\partial \phi}{\partial n}$ be continuous. Since $a$ is a second order quantity, the last of the equations (4) reduced to $\nabla^{2} \phi=0$. Similarly at the boundary between two materials of different magnetic permeability, the continuity of $H_{\mathrm{s}}$ demands that

$$
\frac{1}{\mu} \frac{\partial H}{\partial n}\left(=H_{8}\right)
$$

be continuous. The continuity of $B_{\mathrm{n}}$ is assured by the continuity of $A$ since $B_{\mathrm{n}}=\frac{\partial A}{\partial s}$.

Therefore, the differential equations which $\phi$ and $A$ must satisfy are

(a) $\nabla^{2} \phi=0$ everywhere

(b) $\phi=c_{\mathrm{n}}$ on the section $S_{\mathrm{n}}$ of the $n^{\text {th }}$ conducting group

(c) $\phi$ is continuous everywhere

(d) $a \frac{\partial \phi}{\partial n}$ is continuous at the boundaries between two different dielectrics

(e) $\operatorname{limit}_{r=\infty}\left(r^{2} \frac{\partial \phi}{\partial r}\right)$ exists for all directions of $r$

(a) $\nabla^{2} A=O$ in dielectrics

$$
=4 \pi i p \mu \lambda\left(A-\frac{\gamma \phi}{i p}\right) \text { in conductors }
$$

(b) $A$ is continuous at all boundaries

(c) $\frac{1}{\mu} \frac{\partial A}{\partial n}$ is continuous at all boundaries

(d) $\underset{r=\infty}{\operatorname{limit}}\left(r^{2} \frac{\partial A}{\partial r}\right)$ exists for all directions of $r$ 
The relation (8a) follows from (4). The conditions at infinity (7e) and $(8 d)$ are derived as follows:

Let $I_{\mathrm{n}}$ denote the total $z$ component of current through the section $S_{\mathrm{n}}$ of the $n^{\text {th }}$ conductor (or $n^{\text {th }}$ group of conductors in contact).

The set of closed contours or artificial boundaries, shown by dotted lines in Figure 1, are drawn so that each artificial boundary incloses a homogeneous material. In the case of materials extending to infinity in the $x y$ plane, the artificial boundary is closed by arcs of a circle with center at some finite point and indefinitely large

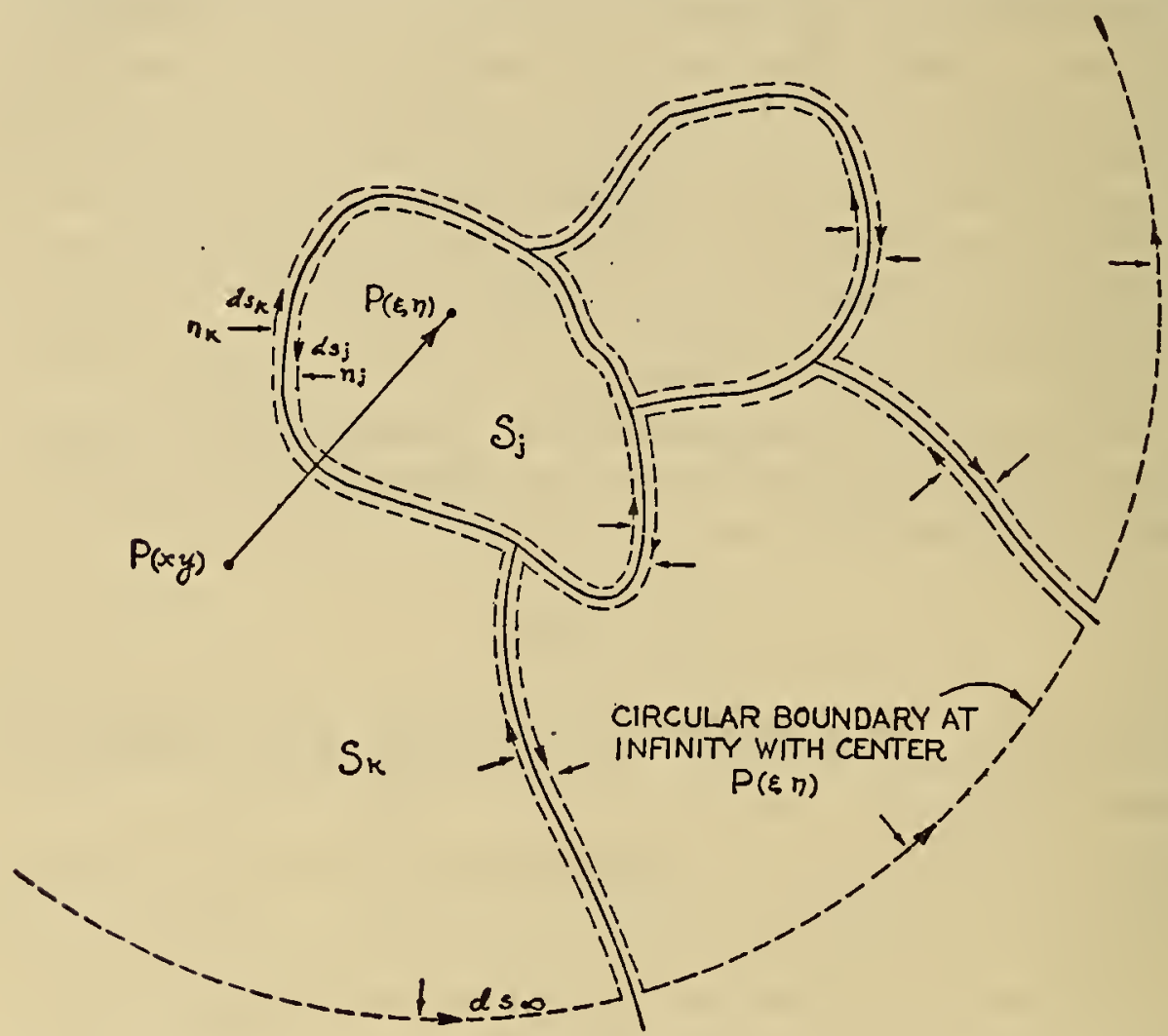

FIG. 1.-Plane section of cylindrical media $(z=$ constant $)$ showing positive directions of normals and line-elements in each region

(Full liaes are natural boundaries. Dotted lines are artificial boundaries)

radius. The natural boundaries are shown by full lines. The normal to the boundary is taken in each medium pointing toward the boundary, and the positive direction of an element ds of an artificial boundary curve is such that in going around this contour in the positive direction the homogeneous medium encircled is on the left hand. Thus the directions of $n$ and $d s$ are related like those of the $x$ and $y$ axes, respectively. With this understanding, the statement of the conservation of electricity applied to the $n^{\text {th }}$ conductor is

$$
\gamma I_{\mathrm{n}}=\int a \frac{\partial \phi}{\partial n} d s_{\mathbf{n}} \quad n=1,2,3 \ldots \ldots N
$$


where the integral is taken around any closed curve in the dielectric which encircles this conductor only, the normal $n$ pointing toward the conductor.

Since $\phi$ and $a \frac{\partial \phi}{\partial n}$ are continuous and $\nabla^{2} \phi=0$ in every dielectric, it follows that $\int a \frac{\partial \phi}{\partial n} d s=0$ taken over any closed curve which does no encircle a conductor. Consequently by (9)

$$
\gamma \sum_{1}^{N} I_{\mathrm{n}}=\sum_{n=1}^{N} \int a \frac{\partial \phi}{\partial n} d s_{\mathrm{n}}=-{ }_{r=\infty}^{\operatorname{limit}} r \int_{0}^{2 \pi} a \frac{\partial \phi}{\partial r} d \Theta
$$

where $r$ is the radius of a circle with some finite point taken as center.

Since the terminal apparatus located in finite portions of the planes $z=0$, and $z=l$ are assumed to have no mutual capacity or coupling with any of these cylinders, it follows that the sum of the currents entering either end must be zero at every instant, so that

$$
\sum_{n=1}^{N} I_{\mathrm{n}}=0
$$

Hence, the ${ }_{r=\infty}^{\text {limit }} r \frac{\partial \phi}{\partial r}$ must be zero. But if we assume that the density of surface change on the natural boundaries is finite and falls off so rapidly as the point on the boundary moves off to infinity that the integral which defines its logarithmic potential is convergent, then it follows that $\frac{\partial \phi}{\partial r}$ must vanish in such a manner that $\operatorname{limit}_{r=\infty} r^{2} \frac{\partial \phi}{\partial r}$ exists which is the condition (7e).

Similarly, it is assumed that the density of current (surface densities of molecular currents on boundaries or magnetic surface charges) are so distributed that their logarithmic potential exists, and if $r \frac{\partial A}{\partial r}$ vanishes it must vanish canonically. The definition of current takes the form

$$
I_{\mathrm{n}}=\frac{1}{4 \pi} \int \frac{\partial A}{\partial n} d s_{\mathrm{n}}
$$

the integral being taken in the dielectric as in (9). A similar reasoning leads to the condition $(8 d)$.

In the next two sections it will appear that the conditions (7) and (8) are necessary and sufficient to uniquely determine $\phi$ and $A$ in terms of the potentials $c_{1} c_{2} \ldots \ldots c_{\mathbb{N}}$. These solutions of (7) and (8) will make $\sum_{1}^{N} I_{n}=O$, which is reciprocal with the requirement that $\int r \frac{\partial \phi}{\partial r}$ and $r \frac{\partial A}{\partial r}$ shall vanish at infinity and in such a manner that $39058^{\circ}-25-2$ 
$\int r^{2} \frac{\partial \phi}{\partial r}$ and $r^{2} \frac{\partial A}{\partial r}$ shall exist. If no conductor extends to infinity, then $\phi$ and $A$ are harmonic and the values of $\phi(\infty)$ and $A(\infty)$ will be determinate, since one can not arbitrarily assign both the value of the harmonic function and of its normal derivative at infinity (if the c's are all arbitrary). In the case where a conductor, say, the $N^{\text {th }}$, extends to infinity, then $A$ and $E$ will not be harmonic in this conductor but will satisfy

since

$$
\nabla^{2} A-4 \pi \text { ipa } A=0=\nabla^{2} E-4 \pi^{n} \text { ipa } E
$$

$$
\phi\left(x_{\mathrm{N}} y_{\mathrm{N}}\right)-\phi(\infty)=1-1=0
$$

if $x_{\mathrm{N}} y_{\mathrm{N}}$ is on this conductor. Consequently, the vanishing of $r \frac{\partial A}{\partial r}$ (and hence $r \frac{\partial E}{\partial r}$ ) brings with it the fact that both $A$ and $E$ mush vanish at infinity.

Whether either of the finite constants $\phi(\infty)$ or $A(\infty)$ have the value zero is a question without physical significance, since the $x$ and $y$ components of electric and magnetic field will vanish properly at infinity. But the linear combination $\gamma \phi(\infty)-i p A(\infty)$ has the physical significance $E(\infty)\left[=E_{z}(\infty)\right]$. Consequently, we must conclude that for the type of wave assumed the first approximations here attempted will give a definite value to $E(\infty)$ which can not be arbitrarily assigned the value zero. As a matter of fact, it will be found that $E(\infty)$ will not have the value zero in the case where one conductor of finite section surrounds all the others. Even in this case the formal solution we shall obtain will give the first approximation-that is, the finite terms-to the current distribution in conductors. A further approximation would not appreciably affect the value of $E$ in conductors but would give a function $E_{\mathrm{z}}$ which is not strictly harmonic in the dielectric and hence which vanishes at infinity since $\frac{\partial E}{\partial r}$ must do so.

In case more than one conductor extends to infinity we shall assume that they are at the same potential and may be treated as one group of conductors in contact. This avoids the case where two conductors have infinite coefficients of capacity or leakage per unit length.

\section{THE COMPLEX SCALAR POTENTIAL $\phi$}

\section{UNIQUENESS OF A SOLUTION}

If the complex values $c_{1}, c_{2}, \ldots c_{\mathrm{N}}$ which $\phi$ must assume on the conductors are arbitrarily assigned, there can not be more than one function $\phi$ satisfying the conditions (7). If there were another, their difference $\phi^{\prime}+i \phi^{\prime \prime}$ would satisfy all these conditions and van- 
ish on each conductor, where $\phi^{\prime}$ and $\phi^{\prime \prime}$ are real. The boundary conditions ( $7 d$ ) imply the two real relations

$$
\begin{aligned}
\lambda_{1} \frac{\partial \phi^{\prime}}{\partial n_{1}}+\lambda_{2} \frac{\partial \phi^{\prime}}{\partial n_{2}} & =\frac{p}{4 \pi c^{2}}\left(k_{1} \frac{\partial \phi^{\prime \prime}}{\partial n_{1}}+k_{2} \frac{\partial \phi^{\prime \prime}}{\partial n_{2}}\right) \\
\lambda_{1} \frac{\partial \phi^{\prime \prime}}{\partial n_{1}}+\lambda_{2} \frac{\partial \phi^{\prime \prime}}{\partial n_{2}} & =-\frac{p}{4 \pi c^{2}}\left(k_{1} \frac{\partial \phi^{\prime}}{\partial n_{1}}+k_{2} \frac{\partial \phi^{\prime}}{\partial n^{2}}\right)
\end{aligned}
$$

Also $\phi^{\prime}$ and $\phi^{\prime \prime}$ are harmonic within each dielectric section $S_{\mathrm{j}}$ and

$$
\operatorname{limit}_{r=\infty} \int_{0}^{2 \pi} \frac{\partial \phi^{\prime}}{\partial r} r d \theta=\frac{\operatorname{limit}}{r=\infty} \int_{0}^{2 \pi} \frac{\partial \phi^{\prime \prime}}{\partial r} r d \theta=0
$$

and

$$
\int\left(\phi^{\prime} \frac{\partial \phi^{\prime \prime}}{\partial n}-\phi^{\prime \prime} \frac{\partial \phi^{\prime}}{\partial n}\right) d s_{\mathbf{j}}=0
$$

taken around the artificial boundary of $S_{\mathrm{j}}$ or only around that part of it which is adjacent to another dielectric since $\phi^{\prime}$ and $\phi^{\prime \prime}$ vanish at conducting boundaries, and the integral vanishes for arcs of the circle at infinity.

By the use of Green's theorem it is easy to show that

$$
\begin{gathered}
\iint \lambda\left[\left(\frac{\partial \phi^{\prime}}{\partial x}\right)^{2}+\left(\frac{\partial \phi^{\prime}}{\partial y}\right)^{2}+\left(\frac{\partial \phi^{\prime \prime}}{\partial x}\right)^{2}+\left(\frac{\partial \phi^{\prime \prime}}{\partial y}\right)^{2}\right] d S_{j} \\
=\int \lambda\left[\phi^{\prime} \frac{\partial \phi^{\prime}}{\partial n}+\phi^{\prime \prime} \frac{\partial \phi^{\prime \prime}}{\partial n}\right] d s_{\mathbf{j}} .
\end{gathered}
$$

Summing all such equations for the entire dielectric region of the $x y$ plane gives

$$
\begin{aligned}
\iint \lambda\left[\left(\frac{\partial \phi^{\prime}}{\partial x}\right)^{2}\right. & \left.+\left(\frac{\partial \phi^{\prime}}{\partial y}\right)^{2}+\left(\frac{\partial \phi^{\prime \prime}}{\partial x}\right)^{2}+\left(\frac{\partial \phi^{\prime \prime}}{\partial y}\right)^{2}\right] d s \\
& =\sum_{j} \int \lambda\left[\phi^{\prime} \frac{\partial \phi^{\prime}}{\partial n}+\phi^{\prime \prime} \frac{\partial \phi^{\prime \prime}}{\partial n}\right] d s_{j}
\end{aligned}
$$

where the double integral on the left extends over the entire dielectric region, the line integrals on the right are taken around all the closed artificial boundaries of all the dielectrics. The terms corresponding to the conducting part of these boundaries vanish because $\phi^{\prime}$ and $\phi^{\prime \prime}$ are zero there. The terms corresponding to the boundaries between two dielectrics may be written by virtue of the above boundary conditions in the form

$$
\frac{p}{4 \pi c^{2}} \int k\left(\phi^{\prime} \frac{\partial \phi^{\prime \prime}}{\partial n}-\phi^{\prime \prime} \frac{\partial \phi^{\prime}}{\partial n}\right) d s
$$

over both sides of all the artificial boundaries between two dielectrics, and this has been shown to be zero. Hence, $\phi^{\prime}$ and $\phi^{\prime \prime}$ must be con- 
stant over the entire dielectric region, since they are continuous there. They vanish on conductors and hence $\phi^{\prime}=\phi^{\prime \prime}=0$, which shows that there can not be more than one function $\phi$ which satisfies the conditions (7).

\section{EXISTENCE AND UNIQUENESS OF A GENERALIZED GREEN'S FUNCTION G}

To find whether it is possible for any function $\phi$ to exist which satisfies the condition (7), and if so to obtain an integral representation of $\phi$, we may make use of a generalization of Green's function $G(x y \xi \eta)$, which may be regarded as the potential at any point $x y$ in the dielectric region, when there is a line source of unit strength (per unit length along $z$ ) which is parallel to $z$, at some fixed point $\xi \eta$ in the dielectric region, and when all the conductors are kept at zero potential and all together receive the unit current from the line source.

The properties of this function are

(a) $\left(\frac{\partial^{2}}{\partial x^{2}}+\frac{\partial^{2}}{\partial y^{2}}\right) G(x y \xi \eta)=0$ where $x y$ is a point within any dielectric section.

(b) $G(x y \xi \eta)$ and $a(x y) \frac{\partial}{\partial n} G(x y \xi \eta)$ change continuously when the point $x y$ moves across the boundary line between two dielectrics.

(c) $G(x y \xi \eta)$ vanishes when the point $x y$ moves up to a conducting boundary of the dielectric region.

(d) When the point $x y$ approaches the fixed point $\xi \eta, G(x y \xi \eta)$ becomes infinite in such a manner that it differs from $-\frac{2 \log r(x y \xi \eta)}{4 \pi a(\xi \eta)}$ by a finite quantity. The strength of the source is $\int a \frac{\partial G(x y \xi \eta)}{\partial n} d s$ taken around an infinitesimal circle with center at $\xi \eta$, and is thus equal to 1 , if $m$ is drawn toward the circle, on the outside. This represents the efflux of electricity from the line source per second per unit length along $z$ (leakage and displacement current together).

(e) When the dielectric region extends to infinity, if $x y$ moves off to infinity in any direction in the $x y$ plane $(\xi \eta$ being a fixed finite point), then $\frac{\partial G}{\partial r}$ vanishes (so that the ${\underset{r=\infty}{r=\infty}}^{2} \frac{\partial G}{\partial r}(x y \xi \eta)$ exists). Hence, $G$ takes on the asymptotic form $G(x y \xi \eta)=\mathrm{G}(\infty \xi \eta)+$ terms in $\frac{1}{r}$ and higher. The constant $G(\infty \xi \eta)$ is calculable, not assignable. From these properties it is easily shown by applying Green's theorem to the two functions $G\left(x y x^{\prime} y^{\prime}\right)$ and $G\left(\xi \eta x^{\prime} y^{\prime}\right)$, where $x y$ and $\xi \eta$ are any two distinct points whatever in the dielectric region, 
that this function is a symmetrical one of the two points; that is

$$
G(x y \xi \eta)=G(\xi \eta x y)
$$

This means that the potential at $x y$ due to a line charge at $\xi \eta$ will have the same value as the potential at $\xi \eta$ due to a line charge at $x y$. From this interpretation it is evident that $\left(\frac{\partial^{2}}{\partial \xi^{2}}+\frac{\partial^{2}}{\partial \eta^{2}}\right) G=0$ and that if $x y$ is a fixed point and $\xi \eta$ the variable, then $G$ and $a \frac{\partial G}{\partial n}$ will be continuous.

If we apply the integral transformation

$$
\iint a \nabla^{2} G d S_{\mathrm{j}}=\int a \frac{\partial G}{\partial n} d s_{\mathrm{j}}
$$

to each homogeneous dielectric section $S_{\mathrm{j}}$ and sum for all of them, excluding the point $\xi \eta$ by an infinitesimal circle, we find that

$$
\sum_{k=1}^{N} \int a \frac{\partial G}{\partial n_{\mathrm{k}}}\left(x_{\mathrm{k}} y_{\mathrm{k}} \xi \eta\right) d s_{\mathrm{k}}=-1
$$

where the $k^{\text {th }}$ integral is taken around any closed curve in the dielectric which encircles the $k^{\text {th }}$ conducting group only. This merely states the fact that the total flow from the line source goes into the conductors and there is no flow to infinity.

To prove the existence and uniqueness of such a function $G$ having the desired properties, we may begin by assuming that a Green's function $g(x y \xi \eta)$ has been constructed for the entire dielectric region as if it were homogeneous. In the case of a closed finite region, this is usually effected by writing

$$
g(x y \xi \eta)=-\frac{1}{2 \pi} \log r(x y \xi \eta)+v(x y \xi \eta)
$$

and choosing $v(x y \xi \eta)$ as a function which satisfies $\frac{\partial^{2} v}{\partial x^{2}}+\frac{\partial^{2} v}{\partial y^{2}}=0$ in the region, and assumes the value $\frac{1}{2 \pi} \log r\left(x^{\prime} y^{\prime} \xi \eta\right)$ when $x y$ approaches the point $x^{\prime} y^{\prime}$ on the boundary, thus making $g(x y \xi \eta)$ vanish when $x y$ approaches the boundary point $x^{\prime} y^{\prime}$. The existence and uniqueness of $v$ is therefore a special case of the more general problem of Dirichlet of finding a harmonic function which assumes any assigned boundary values. Since the development of the theory of Fredholm's integral equation it may be taken as satisfactorily proven that a harmonic function exists and is unique if either

or

(a) Its value is assigned on all points of the boundary

(b) Its value is assigned on some parts of the boundary, and the values of its normal derivative on all remaining parts. ${ }^{2}$

2 Cf. Volterra. Lecons sur les Equations Integrals, p. 126. Max Mason “Boundary Value Problems. New Haven Math. Colloquium " 
In the case of an unbounded region, the value of $g(x y \xi \eta)$ when $x y$ goes to infinity is not prescribed, but the condition is imposed that $\operatorname{limit}_{r=\infty} r^{2} \frac{\partial \phi}{\partial r}$ shall exist. This, together with the facts that $g$ shall be harmonic in $x y$ and vanish on all the internal boundaries of the region and become logarithmically infinite when $x y$ approaches $\xi \eta$, serves to uniquely determine $g$ and hence determines the value which it will assume at infinity.

If we apply Green's theorem to the two functions $a(x y) G\left(x y \xi_{1} \eta_{1}\right)$ and $a(x y) g(x y \xi \eta)$ the point $x y$ being the variable of integration, we find if $\xi_{1} \eta_{1}$ is within the homogeneous dielectric section $S_{\mathrm{j}}$ and $\xi_{\eta}$ in $S_{\mathrm{k}}$

$-g\left(\xi_{1} \eta_{1} \xi \eta\right)=$

$$
-\int a\left[G\left(x y \xi_{1} \eta_{1}\right) \frac{\partial g(x y \xi \eta)}{\partial n}-g(x y \xi \eta) \frac{\partial G\left(x y \xi_{1} \eta_{1}\right)}{\partial n}\right] d s_{\mathrm{j}}
$$

$a(\xi \eta) G\left(\xi \eta \xi_{1} \eta_{1}\right)=$

$$
-\int a\left[G\left(x y \xi_{1} \eta_{1}\right) \frac{\partial g(x y \xi \eta)}{\partial n}-g(x y \xi \eta) \frac{\partial G\left(x y \xi_{1} \dot{\eta}_{1}\right)}{\partial n}\right] d s_{\mathbf{k}}
$$

the integrals bcing taken around the artificial contours of the corresponding dielectric section. The contour integral on the right has the value zero if taken around any other artificial boundary of a dielectric. Hence, adding all such terms to cover the entire dielectric region we get

$$
a(\xi \eta) G\left(\xi \eta \xi_{1} \eta_{1}\right)+\mathcal{S}\left[a_{1}(s)-a_{2}(s)\right] \frac{\partial g(s \xi \eta)}{\partial n_{1}} G\left(s \xi_{1} \eta_{1}\right) d s=g\left(\xi_{1} \eta_{1} \xi \eta\right)
$$

where the line integral is taken once over all the boundaries between different dielectrics, the point $s(x y)$ being the point of integration on this curve. The values $a_{1}(s)$ and $a_{2}(s)$ correspond to the medium on the left and right of the curve, respectively, at the point $s$. The positive direction of $d s$ may be arbitrary, but the direction of the normal $n$ must be such that $n_{1}$ and $d_{1}$ are related to each other like the directions of the $x$ and $y$ axes, respectively.

The continuity of $G, a \frac{\partial G}{\partial n}, g$ and $\frac{\partial g}{\partial n}$ have been used in obtaining this equation. (The point $s(x y)$ on the boundary $s$ is an ordinary point for Green's function $g$ ).

If the point $(\xi \eta)$ now approaches a point $s^{\prime}\left(x^{\prime} y^{\prime}\right)$ on the path of the line integral from the left side the equation becomes

or

$$
\begin{gathered}
a_{1}\left(s^{\prime}\right) G\left(s^{\prime} \xi_{1} \eta_{1}\right)-\left[\frac{a_{1}\left(s^{\prime}\right)-a_{2}\left(s^{\prime}\right)}{2}\right] G\left(s^{\prime} \xi_{1} \eta_{1}\right) \\
+\int\left[a_{1}(s)-a_{2}(s)\right] \frac{\partial g}{\partial n_{1}}\left(s s^{\prime}\right) G\left(s \xi_{1} \eta_{1}\right) d s=g\left(\xi_{1} \eta_{1} x^{\prime} y^{\prime}\right)=g\left(\xi_{1} \eta_{1} s^{\prime}\right)
\end{gathered}
$$

$G\left(s^{\prime} \xi_{1} \eta_{1}\right)+2 \int \frac{a_{1}(s)-a_{2}(s)}{a_{1}\left(s^{\prime}\right)+a_{2}\left(s^{\prime}\right)} \frac{\partial g}{\partial n_{1}}\left(s s^{\prime}\right) G\left(s \xi_{1} \eta_{1}\right) d s=\frac{2 g\left(\xi_{1} \eta_{1} s^{\prime}\right)}{a_{1}\left(s^{\prime}\right)+a_{2}\left(s^{\prime}\right)}$. 
The same result is obtained when $\xi \eta$ approaches $s^{\prime}\left(x^{\prime} y^{\prime}\right)$ from the right, since the contribution of the infinitesimal element of the line integral at $s^{\prime}$ is now

$$
+\frac{a_{1}\left(s^{\prime}\right)-a_{2}\left(s^{\prime}\right)}{2} G\left(s^{\prime} \xi_{1} \eta_{1}\right)
$$

This is found from the fact that the principal part of $\frac{\partial g}{\partial n_{1}}(s \xi \eta)$ becomes $\frac{\partial \theta(s \xi \eta)}{\partial s_{1}}$.

If the characteristic determinant of the integral equation (15) does not vanish, it suffices to uniquely determine $G\left(s \xi_{1} \eta_{1}\right)$ at all points $s$ on the boundary curve, and hence by (3) at all points in the dielectric region. To prove that this determinant can not vanish, we make use of the fact that if it does there must be at least one solution $G_{0}\left(s \xi_{1} \eta_{1}\right)$ not identically zero of the homogeneous integral equation obtained by replacing the second member of (15) by zero; that is,

$$
G_{0}\left(s^{\prime} \xi_{1} \eta_{1}\right)+2 \int \frac{a_{1}(s)-a_{2}(s)}{a_{1}\left(s^{\prime}\right)+a_{2}\left(s^{\prime}\right)} \frac{\partial g}{\partial n_{1}}\left(s s^{\prime}\right) G_{0}\left(s \xi_{1} \eta_{1}\right) d s=0 .
$$

But if this were the case we could construct a scalar potential function $\phi_{\mathrm{o}}(\xi \eta)$ for any point $\xi \eta$ within the dielectric region by the formula

$$
\phi_{0}(\xi \eta)=-\frac{1}{a(\xi \eta)} \int\left[a_{1}(s)-a_{2}(s)\right] \frac{\partial g}{\partial n_{1}}(s \xi \eta) G_{0}\left(s \xi_{1} \eta_{1}\right) d s
$$

which could not be identically zero in all parts of the plane.

From the property of Green's function $g$, it is evident that $\phi_{0}(\xi \eta)$ will vanish when $\xi \eta$ moves up to any conducting boundary, will be harmonic in $\xi \eta$ at all ordinary points. Also it is evident that $a$ $\frac{\partial \phi_{\mathrm{o}}}{\partial n}$ will be continuous at the boundaries between dielectrics, and $\operatorname{limit}_{r=\infty}\left(r^{2} \frac{\partial \phi_{o}}{\partial r}\right)$ will exist. Finally, if $\xi \eta$ approaches a point on the boundary between two dielectrics, then the homogeneous integral equation (16), which $G_{\mathrm{o}}$ is assumed to satisfy, will show that $\phi_{\mathrm{o}}$ also is continuous there. Thus $\phi_{\mathrm{o}}$ possesses all the properties which have been shown sufficient to assure its nonexistence. Therefore, it may be concluded that a function $G(x y \xi \eta)$ and one only may be found.

\section{THE EXISTENCE OF $\phi$ AND ITS INTEGRAL REPRESENTATION}

Assuming that the function of $G$ has been constructed, we may apply Green's theorem to the two functions $\phi(x y)$ and $G(x y \xi \eta)$, and obtain

$$
--\int a_{\mathfrak{S}}\left[\phi(x y) \frac{\partial G(x y \xi \eta)}{\partial n}-G(x y \xi \eta) \frac{\partial \phi(x y)}{\partial n}\right] d s_{\mathrm{j}}=\phi(\xi \eta)
$$


if $\xi \eta$ is a point within the homogeneous dielectric section $S_{\mathrm{j}}$, the integration being taken around the artificial boundary of $S_{j}$. Applied to any other dielectric section $S$, the theorem gives

$$
-\int a\left[\phi(x y) \frac{\partial G(x y \xi \eta)}{\partial n}-G(x y \xi \eta) \frac{\partial \phi(x y)}{\partial n}\right] d s=0 .
$$

Adding the first expression to all the others of the second type so as to include the entire dielectric region, it will be found that all the integrals over the boundaries between two dielectrics cancel, because of the continuity of $\phi$ and $a \frac{\partial \phi}{\partial n}$ on the one hand and of $G$ and $a \frac{\partial G}{\partial n}$ on the other. Also the integrals $G(x y \xi \eta) \frac{\partial \phi}{\partial n}$ over the conducting boundaries of the region vanish because $G$ vanishes there. The integrals over the infinite circle vanish because $r \frac{\partial \phi}{\partial r}$ and $r \frac{\partial G}{\partial r}$ vanish at infinity.

Hence, we obtain the integral representation

$$
\begin{aligned}
\phi(\xi \eta) & =-\sum_{k=1}^{N} c_{\mathrm{k}} \int a(x y) \frac{\partial G(x y \xi \eta)}{\partial n_{\mathrm{k}}} d s_{\mathbf{k}} \\
& =\sum_{k=1}^{N} c_{\mathrm{k}} \phi_{\mathrm{k}}(\xi \eta)
\end{aligned}
$$

where

$$
\phi_{\mathrm{k}}(\xi \eta) \equiv-\int a(x y) \frac{\partial G(x y \xi \eta)}{\partial n_{\mathrm{k}}} d s_{\mathrm{k}}
$$

this integral being taken around any closed contour in the dielectric region which surrounds the $k^{\text {th }}$ group of conductors only.

The $N$ equations of type (9), for the conservation of electricity give $N$ equations of the type

$$
\gamma I_{\mathrm{n}}=\int a \frac{\partial \phi}{\partial n_{\mathrm{n}}} d s_{\mathrm{n}}=\sum_{k=1}^{N} c_{\mathrm{k}} \int a \frac{\partial \phi_{\mathrm{k}}}{\partial n_{\mathrm{n}}} d s_{\mathrm{n}}=\sum_{k=1}^{N} a_{\mathrm{nk}} c_{\mathbf{k}}
$$

where the complex coefficient $\alpha_{\mathrm{nk}}$ is defined by

$$
a_{\mathrm{nk}} \equiv \int a \frac{\partial \phi_{\mathrm{k}}}{\partial n_{\mathrm{n}}} d s_{\mathrm{n}}
$$

where this integral is taken over any closed curve in the dielectric region which encircles the $n^{\text {th }}$ group of conductors only. From this defraition and the definition (8) of $\phi_{\mathrm{k}}$ it follows that the coefficients $a_{\mathrm{nk}}$ constitute a symmetrical array, that is

$$
a_{\mathrm{nk}}=a_{\mathrm{kn}}
$$

This is seen by inserting the definition (19) of $\phi_{\mathrm{k}}$ in (21), letting $x y$ denote a point on or near the boundary of the conducting section $S_{n}$ and $\xi \eta$ a point on or near the boundary of the conducting section $S_{\mathrm{k}}$

$$
a_{\mathrm{nk}}=-\iint d s_{\mathrm{n}} d s_{\mathrm{k}} a(x y) a(\xi \eta) \frac{\partial^{2} G(x y \xi \eta)}{\partial n_{\mathrm{n}} \partial n_{\mathrm{k}}} .
$$


Since $G\left(x y \xi_{\eta}\right)=G\left(\xi_{\eta} x y\right)$ this is easily seen to be identical with the definition of $a_{\mathrm{kn}}$.

The unique solution or integral representation (18) for $\phi(\xi \eta)$ has been obtained by assuming a function $\phi$ to exist which satisfies all the conditions in (7) of Section II. It remains to be shown that the function $\phi\left(\xi_{\eta}\right)$ which is given by the right side of (19) does indeed satisfy all these conditions, and that if each of the $N$ currents $I_{1} I_{2} \ldots I_{\mathrm{N}}$ satisfies the equation of conservation of electricity (20), the sum of these currents will be identically zero, whatever the value of the $N$ constant potentials $c_{1} c_{2} \ldots c_{\mathrm{N}}$ or of $\gamma$.

To do this and to obtain an idea of their physical meaning we may examine the $N$ partial potentials of type $\phi_{\mathrm{k}}(\xi \eta)$ defined by (19).

\section{PROPERTIES AND PHYSICAL INTERPRETATION OF THE PARTIAL POTENTIALS $\phi_{\mathrm{n}}$}

From the properties of $G(x y \xi \eta)$ it follows that $\phi_{\mathrm{k}}\left(\xi_{\eta}\right)$ has the following properties

$$
\left(\frac{\partial^{2}}{\partial \xi^{2}}+\frac{\partial^{2}}{\partial \eta^{2}}\right) \phi_{\mathrm{k}}(\xi \eta)=0
$$

if $\xi \eta$ is any point not on a boundary. This is evident by differentiating under the integral sign twice with respect to $\xi$ and to $\eta$ which is allowable since $\xi_{\eta}$ is not a point on the line of integration. Since

it follows that

$$
\left(\frac{\partial^{2}}{\partial \xi^{2}}+\frac{\partial^{2}}{\partial \eta^{2}}\right) G(x y \xi \eta)=0
$$

$$
\frac{\partial^{2} \phi_{\mathrm{k}}}{\partial \xi^{2}}+\frac{\partial \phi_{\mathrm{k}}}{\partial \eta^{2}}=0
$$

(b) When $\xi_{\eta}$ moves across any boundary between two dielectrics $\phi_{\mathrm{k}}\left(\xi_{\eta}\right)$ changes continuously because $G(x y \xi \eta)$ is also a continuous function of $\xi_{\eta}$ for any fixed value of $x y$.

(c) At such boundaries $a \frac{\partial \phi_{\mathrm{k}}}{\partial n}$ is also continuous because of the similar continuity of $a \frac{\partial G(x y \xi \eta)}{n}$ when $x y$ is any fixed point.

(d) The $\operatorname{limit}_{r=\infty}\left(r^{2} \frac{\partial \phi_{\mathrm{k}}}{\partial r}\right)$ exists when $\xi_{\eta}$ goes to infinity in any direction in the $x y$ plane, because of the similar property of $G(x y \xi \eta)$ when $x y$ is a finite point.

(e) When $\xi_{\eta}$ moves off to infinity in any direction in the $x y$ plane $\phi_{\mathrm{k}}\left(\xi_{\eta}\right)$ will become constant, say $\phi_{\mathrm{k}}(\infty)$, which will not necessarily be zero. From the definition (8) it is evident that $\phi_{\mathrm{k}}\left(\xi_{\eta}\right)$ is equal to the total conduction and displacement current (per unit length along $z$ ) which flows into the $k^{\text {th }}$ conductor, from the unit line $39058^{\circ}-25-3$ 
source at $\xi \eta$, when all the conductors are at zero potential and all together receive the total current unity, from the line source. It is thus evident that if these conditions are satisfied, that $\phi_{\mathbf{k}}(\xi \eta)$ will not, in general, vanish when $\xi \eta$ goes to infinity. If there is one conductor extending to infinity, the $N^{\text {th }}$ say, then $\phi_{N}(\infty)$ will be unity and $\phi_{\mathrm{n}}(\infty)=0$, if $n \neq N$, since the infinite conductor will in this case receive all of the current from the line source at $\xi \eta$ when the latter moves to infinity. The same statements are true if $\phi_{\mathrm{N}}$ surrounds all the others.

(f) When the point $\xi_{\eta}$ approaches any conducting section $S_{\mathrm{n}}$, then $\phi_{\mathbf{V}}(\xi \eta)$ approaches zero if $n \neq k$ and $\phi_{\mathbf{k}}(\xi \eta)$ approaches unity at the boundary of conducting section $S_{\mathrm{k}}$. This is evident from the above interpretation of $\phi_{\mathrm{k}}(\xi \eta)$ as the current into $S_{\mathrm{k}}$ when there is a unit line source at $\xi \eta$. When $\xi \eta$ comes infinitely close to the boundary of any conductor, the unit current will all flow from the line source into the conductor in its immediate neighborhood and hence the flow into any other conductor will be zero.

Thus $\phi_{\mathrm{k}}$ possesses all the properties which we have shown are necessary to make it unique and it may be determined by the methods of harmonic analysis.

From these properties of each $\phi_{\mathrm{k}}$ it is evident that the function $\phi(\xi \eta)$ given by (18) does satisfy all the conditions (7) Section II. It is the only one which exists.

The $N$ functions $\phi_{1} \phi_{2} \ldots \phi_{\mathrm{N}}$ are not all independent for there exists a linear relation between them:

$$
\sum_{k=1}^{N} \phi_{\mathrm{k}}(\xi \eta)=1
$$

This also follows from the interpretation of $\phi_{\mathrm{k}}(\xi \eta)$ as current into the section $S_{\mathrm{k}}$, since altogether the $N$ conducting sections receive the current unity from the line source at $\xi \eta$, equations (19) and (13). From (23) it follows that there are $N$ homogeneous linear relations existing between the coefficients $\alpha_{\mathrm{n} \text { r. }}$. Thus operating on (23) by $\int a \frac{\partial}{\partial n_{\mathrm{n}}} d s_{\mathrm{n}}$ gives

$$
\sum_{n=1}^{N} \alpha_{\mathrm{nk}}=0 \text { for } k=1,2,3, \ldots N
$$

From this it is evident that $I_{1}+I_{2}+\ldots+I_{\mathrm{N}}=0$ identically whatever the value of $c_{1}, c_{2}, \ldots c_{\mathrm{N}}$ and $\gamma$. The last of the $N$ equations (20) is not independent of the first $N-1$ equations, but may be derived from them by simply adding them together. The determi- 
nant of this array of coefficients vanishes and it is not possible to solve this set of equations for all the c's in terms of the I's.

Hence, when each $\phi_{\mathrm{k}}$ has been found, then if each of the $N$ currents satisfies the equation of conservation of electricity (20) the necessary relation (10), that the sum of all the currents shall vanish, will be automatically satisfied.

The physical meaning of $\phi_{\mathrm{k}}(\xi \eta)$ follows from its properties. It represents the potential at any point $(\xi \eta)$ when the $k^{\text {th }}$ conductor is at unit potential and all the others at zero potential in the presence of the various conducting dielectrics, subject to the additional condition that the whole flow out from the $k^{\text {th }}$ conductor goes into the remaining ones, as there is no flow to infinity in the $x y$ plane. This is the meaning of the relations (23) and (24).

It is evident that $\phi_{\mathrm{K}}$ is, in general, complex on account of the surface charges at the boundaries between two dielectrics. These charges are eliminated from appearance by the use of $G(x y \xi \eta)$ through which function their influence is exerted, so to speak. If the dielectric were entirely homogeneous both as to its conductivity $\lambda$ and its dielectric constant $k$, then the function $\phi_{\mathrm{k}}$ would be a real electrostatic function, which would not involve $\lambda, k$, or $p$, but would depend only upon the geometric configuration of the conducting sections which bound the dielectric region.

More generally, if the ratio

$$
\frac{\lambda_{1}}{\lambda_{2}}=\frac{k_{1}}{k_{2}}=\frac{a_{1}}{a_{2}}=\frac{\lambda_{1}+\frac{i p k_{1}}{4 \pi c^{2}}}{\lambda_{2}+\frac{i p k_{2}}{4 \pi c^{2}}}
$$

is real, for every pair of dielectrics in contact, then each $\phi_{\mathrm{K}}$ is real, and involves the values of these real ratios, but not the frequency. In particular, if all the dielectrics are nonconducting, this ratio is real and $\phi_{\mathrm{k}}$ will not involve the frequency.

In general, the surface charges and their phases, at the boundaries between different dielectrics, although they are eliminated from consideration, are the cause of $\phi$ being complex and involving the frequency.

It is evident that if a real formula for $\phi_{\mathrm{k}}$ can be obtained for the case of steady flow through the given dielectrics, with given conductivities $\lambda_{1} \lambda_{2} \ldots \ldots$ etc., then the complex solution $\phi_{\mathrm{k}}$ may be obtained by substituting in this expression the corresponding complex conductivities $a_{1} a_{2} \ldots \ldots$ in place of their real values.

$\mathrm{It}$ is to be noted that $\phi_{\mathrm{k}}$ does not involve $\gamma$. 


\section{THE COMPLEX COEFFICIENTS OF LEAKAGE AND CAPACITY}

The complex charge of free electricity $Q_{\mathbf{n}}$ upon, and the leakage current $G_{\mathrm{n}}$ from, the $n^{\text {th }}$ conducting group per unit length along $z$ are given by

$$
\begin{aligned}
& G_{\mathrm{n}}=\int \lambda \frac{\partial \phi}{\partial n_{\mathrm{n}}} d s_{\mathrm{n}}=\sum_{k=1}^{N} c_{k} \int \lambda \frac{\partial \phi_{\mathrm{k}}}{\partial n_{\mathrm{n}}} d s_{\mathrm{n}} \\
& Q_{\mathrm{n}}=\frac{1}{4 \pi c^{2}} \int k \frac{\partial \phi}{\partial n_{\mathrm{n}}} d s_{\mathrm{n}}=\sum_{k=1}^{N} c_{\mathrm{k}} \frac{1}{4 \pi c^{2}} \int k \frac{\partial \phi_{k}}{\partial n_{\mathrm{n}}} d s_{\mathrm{n}} .
\end{aligned}
$$

Consequently, if we resolve the complex coefficient $\alpha_{n k}$ into its real and imaginary components

$$
-\alpha_{\mathrm{nk}}=G_{\mathrm{nk}}+i p C_{\mathrm{nk}} \text { where }\left\{\begin{array}{l}
G_{\mathrm{nk}}=G_{\mathrm{kn}} \text { and } \sum_{k=1}^{N} G_{\mathrm{nk}}=0 \\
C_{\mathrm{nk}}=C_{\mathrm{kn}} \text { and } \sum_{k=1}^{N} C_{\mathrm{nk}}=0
\end{array}\right.
$$

The equation of conservation of electricity (20) takes the form

$$
\gamma I_{\mathrm{n}}=G_{\mathrm{n}}+i p Q_{\mathrm{n}}=-\sum_{k=1}^{N}\left(G_{\mathrm{nk}}+i p C_{\mathrm{nk}}\right) c_{\mathrm{k}} .
$$

By adding to this equation the identically zero quantity

$$
-c_{\mathrm{n}} \sum_{k=1}^{N} \alpha_{\mathrm{nk}}=-\sum_{k=1}^{N} \alpha_{\mathrm{nk}} c_{\mathrm{n}}=\sum_{k=1}^{N}\left(G_{\mathrm{nk}}+i p C_{\mathrm{nk}}\right) c_{\mathrm{n}}
$$

it takes the form

$$
\gamma I_{\mathrm{n}}=G_{\mathrm{n}}+i p Q_{\mathrm{n}}=\sum_{k=1}^{N}\left(G_{\mathrm{nk}}+i p C_{\mathrm{nk}}\right)\left(c_{\mathrm{n}}-c_{\mathrm{k}}\right)
$$

which shows that the real coefficients $G_{\mathrm{nk}}$ and $C_{\mathrm{nk}}$ where $n \neq k$ are coefficien ts of leakage and capacitance, respectively. The coefficients $G_{\mathrm{nn}}$ and $C_{\mathrm{nn}}$ do not occur in the form (28) and may be regarded simply as the negative of the sum of all the other coefficients $G_{n k}$ and $C_{\mathrm{nk}}$, respectively.

If the frequency is very low, or if the entire dielectric region is homogeneous, or more generally whenever the functions $\phi_{\mathrm{n}}$ are real, $G_{\mathrm{nk}}$ and $C_{\mathrm{nk}}$ will not be functions of the frequency and will have their ordinary electrostatic significance since they are then defined by

$$
G_{\mathrm{nk}}=\int \lambda \frac{\partial \phi_{\mathrm{k}}}{\partial n} d s_{\mathrm{n}}, C_{\mathrm{nk}}=\frac{1}{4 \pi c^{2}} \int k \frac{\partial \phi_{\mathrm{k}}}{\partial n} d s_{\mathrm{n}} .
$$

It is evident that, in general, the formula (27) may be put in a form similar to (28), namely,

$$
\gamma I_{\mathrm{n}}=\sum_{k=1}^{N-1} \alpha_{\mathrm{nk}}\left(c_{\mathrm{k}}-c_{\mathrm{N}}\right) \quad \text { for } n=1,2,3, \cdots N .
$$




\section{THE COMPLEX VECTOR POTENTIAL A}

\section{EXISTENCE AND PROPERTIES OF A MAGNETIC FLUX FUNCTION M}

Let $s(x y)$ be any point on the boundary between materials of different permeability. Let $\xi_{\eta}$ and $\xi_{1} \eta_{1}$ be two other points not on this curve. Consider the vector potential function whose $x$ and $y$ components are zero, but whose $z$ component has at the point $\xi \eta$ the value given by the logarithmic potential

$$
M\left(\xi \eta \xi_{1} \eta_{1}\right)=\mu(\xi \eta)\left[-2 \log r\left(\xi \eta \xi_{1} \eta_{1}\right)-2 \int \sigma(s) \frac{\partial \log r(x y \xi \eta)}{\partial n}\right] d s
$$

where the direction of $d s$ along the curve may be taken at pleasure, but that of $d n$ is that of the normal drawn toward the curve in the medium on the left side. The integral represents the value at $\xi \eta$ of the logarithmic potential of a double distribution of strength $\sigma(s)$ upon the boundary curves where $\mu$ is discontinuous. This is harmonic and has continuous normal derivatives $[(\xi \eta)$ being the variable point] at all points. It also vanishes when $\xi \eta$ moves off to infinity. It may also be written in the form

$$
-2 \int \sigma(s) \frac{\partial \log r(x y \xi \eta)}{\partial n} d s=-2 \int \sigma(s) \frac{\partial \theta(x y \xi \eta)}{\partial s} d s
$$

where $\theta(x y \xi \eta)$ is the angle between the positive direction of the $x$ axis and the line drawn from $s(x y)$ to $\xi \eta$.

Regarding $\xi_{1} \eta_{1}$ as the fixed point and $\xi_{\eta}$ variable; it is evident that (whatever the function $\sigma(s)$ so long as the integral is convergent), the function $M\left(\xi \eta \xi_{1} \eta_{1}\right)$ satisfies $\left(\frac{\partial^{2}}{\partial \xi^{2}}+\frac{\partial^{2}}{\partial \eta^{2}}\right) M\left(\xi \eta \xi_{1} \eta_{1}\right)=0$ and takes the form $-2 \mu(\xi \eta) \log r\left(\xi \eta \xi_{1} \eta_{1}\right)$ when $\xi \eta$ goes to infinity, and that $\frac{1}{\mu(\xi \eta)} \frac{\partial}{\partial n}$ $\mathrm{M}\left(\xi \eta \xi_{1} \eta_{1}\right)$ is continuous everywhere. The integral, however, is discontinuous at the boundary curves, but if $\sigma(s)$ can be so chosen as to keep $M\left(\xi \eta \xi_{1} \eta_{1}\right)$ continuous there, then it is evident that $M\left(\xi \eta \xi_{1} \eta_{1}\right)$ will represent a (z-component of) vector potential function at $\xi \eta$ due to a steady unit current filament at $\xi_{1} \eta_{1}$ parallel to $z$, in the presence of all magnetic materials.

This is evident since the normal component of the magnetic induction derived from it is just $\frac{\partial M}{\partial s}$ and this is continuous if $M$ is so. The tangential component $0: B$ divided by $\mu$, that is of $H$, is $-\frac{1}{\mu} \frac{\partial M}{\partial n}$ and this will also be continuous. If we compute the line integral

$$
\int H_{\mathrm{s}} d s=\frac{1}{\mu} \int-\frac{\partial M\left(\xi \eta \xi_{1} \eta_{1}\right)}{\partial n} d s
$$

around an infinitesimal circle with center at $\xi_{1} \eta_{1}$ the result is $4 \pi$. 
To determine the density $\sigma(s)$ we must express the fact that $M\left(\xi \eta \xi_{1} \eta_{1}\right)$ approaches the same value when the point $\xi \eta$ approaches a point $s^{\prime}\left(x^{\prime} y^{\prime}\right)$ on the boundary curve first from the left and then from the right. In the first case the equation gives, if $\mu_{1}\left(s^{\prime}\right)$ is the value on the eft, $\mu_{2}\left(s^{\prime}\right)$ on the right of the curve at $s^{\prime}$

$$
-\frac{M\left(s^{\prime} \xi_{1} \eta_{1}\right)}{2 \mu_{1}\left(s^{\prime}\right)}=\log r\left(s^{\prime} \xi_{1} \eta_{1}\right)+\pi \sigma\left(s^{\prime}\right)+\int \sigma(s) \frac{\left(\partial \theta\left(s s^{\prime}\right)\right.}{\partial s} d s .
$$

In the second case

$$
-\frac{M\left(s^{\prime} \xi_{1} \eta_{1}\right)}{2 \mu_{2}\left(s^{\prime}\right)}=\log r\left(s^{\prime} \xi_{1} \eta_{1}\right)-\pi \sigma\left(s^{\prime}\right)+\int \sigma(s) \frac{\partial \theta\left(s s^{\prime}\right)}{\partial s} d s .
$$

Eliminating $M\left(s^{\prime} \xi_{1} \eta_{1}\right)$ gives the integral equation

$\sigma\left(s^{\prime}\right)+{ }_{\pi}^{1} \int\left(\frac{\mu_{1}-\mu_{2}}{\mu_{1}+\mu_{2}}\right)_{s^{\prime}} \frac{\partial \theta\left(s s^{\prime}\right)}{\partial s} \sigma(s) d s=-\frac{1}{\pi}\left(\frac{\mu_{1}+\mu_{2}}{\mu_{1}-\mu_{2}}\right)_{s^{\prime}} \log r\left(s^{\prime} \xi_{1} \eta_{1}\right)$

to determine the density of the double distribution $\sigma(s)$. The nucleus of this equation becomes infinite when $s=s^{\prime}$, but is integrable, and the equation may be reduced to one with a finite and continuous nucleus by multiplying by this nucleus and integrating over the range. This equation uniquely determines the function $\sigma$ provided the Fredholm determinant is not zero.

That this can not be the case may be proven from the known fact that when this determinant vanishes there is at least one solution $\sigma_{o}(s)$, not identically zero, of the homogeneous equation obtained by placing the right side of the above equation equal to zero. With this function $\sigma_{0}(s)$ we could then form the potential $v_{0}(\xi \eta)$ for every point $\xi \eta$ by the formula

$$
\begin{aligned}
v_{0}(\xi \eta) & =-2 \mu(\xi \eta) \int \sigma_{0}(s) \frac{\partial \log r(x y \xi \eta)}{\partial n} d s \\
& =-2 \mu(\xi \eta) \int \sigma_{0}(s) \frac{\partial \theta(x y \xi \eta)}{\partial s} d s .
\end{aligned}
$$

This function $v_{0}$ would satisfy $\left(\frac{\partial^{2}}{\partial \xi^{2}}+\frac{\partial^{2}}{\xi \eta^{2}}\right) v_{0}=0$ at all points, and $\frac{1}{\mu} \frac{\partial v_{o}}{\partial n}$ would be continuous everywhere and would vanish at infinity. In addition, $v_{0}$ itself would be continuous because of the assumed property of the function $\sigma_{\mathrm{o}}(s)$. Moreover, $v_{\mathrm{o}}$ could not be identically zero in all sections of the plane. It is easy to show that in this case by transformations similar to those previously used that

$$
\iint \frac{1}{\mu}\left[\left(\frac{\partial v_{0}}{\partial \xi}\right)^{2}+\left(\frac{\partial v_{0}}{\partial \eta}\right)^{2}\right] d \xi d \eta=0
$$

the integral being taken over the entire plane, and since $\mu$ is a real positive value everywhere, and $v_{0}$ is real, it is evident that $v_{0}$ must be a constant everywhere, and hence zero since it vanishes at infinity. 
Therefore, the Fredholm determinant does not vanish and there is a unique solution $\sigma(s)$ and the function $M\left(\xi \eta \xi_{1} \eta_{1}\right)$ may be uniquely determined.

By applying Green's theorem to the two functions $M(x y \xi \eta)$ and $M\left(x y \xi_{1} \eta_{1}\right)$ we find that $M$ is a symmetrical function of the two points $x y$ and $\xi_{\eta}$ in whatever magnetic media the two points may be; That is

$$
M(x y \xi \eta)=M(\xi \eta x y) .
$$

In the special case where all the materials in space have the same magnetic permeability $M(x y \xi \eta)$ reduces to $-2 \mu \log r(x y \xi \eta)$.

\section{INTEGRAL EQUATIONS FOR A AND E}

By means of the function $M$ we may formulate the conditions (8) for $A$ as an integral equation involving surface integrals over the conducting sections, but free from boundary terms.

To do this apply Green's theorem to the two functions $A(x y)$ and $M\left(x y \xi_{\xi}\right)$ where $\xi \eta$ is some point within the section $S_{\mathrm{j}}$ which may be either a dielectric or conducting section. This point $\xi \eta$ being excluded by an infinitesimal circle, the theorem gives

$$
\begin{aligned}
-\frac{1}{4 \pi} \iint \frac{M(x y \xi \eta) \nabla^{2} A}{\mu} & d S+\frac{1}{4 \pi} \int \frac{1}{\mu}\left[M(x y \xi \eta) \frac{\partial A}{\partial n}-A \frac{\partial M(x y \xi \eta)}{\partial n}\right] d s \\
& =A(\xi \eta) \text { if } \xi \eta \text { is within } S_{\mathrm{j}} \\
& =0 \text { for all other sections. }
\end{aligned}
$$

Adding together all such expressions corresponding to the entire $x y$ plane, the line integrals over both sides of all boundaries between different magnetic media cancel, because of the continuity of $A, M, \frac{1}{\mu} \frac{\partial A}{\partial n}$ and $\frac{1}{\mu} \frac{\partial M}{\partial n}$. The integral over the infinite circle gives the term $A(\infty)$. (However, it is important to notice at this point that the same result would have been obtained if $A$ were assumed to take the asymptotic form $A=A(\infty)+A^{\prime} \log r$ at infinity.) Replacing $-\frac{\nabla^{2} A}{4 \pi \mu}$ by its value $-\lambda(i p A-\gamma \phi)$ from (8a) of Section II, gives, after interchanging the notation of the points $x y$ and $\xi \eta$

$$
A(x y)+i p \iint M(x y \xi \eta) \lambda(\xi \eta)\left[A(\xi \eta)-\frac{\gamma \phi(\xi \eta)}{i p}\right] d \xi d \eta=A(\infty)
$$

Or since $E=\gamma \phi-i p A$ by (6)

$$
\begin{gathered}
E(x y)+i p \mathcal{S} \mathcal{S} M(x y \xi \eta) \lambda(\xi \eta) E(\xi \eta) d \xi d \eta=\gamma \phi(x y)-i p A(\infty) \\
\equiv F(x y) \equiv \gamma[\phi(x y)-\phi(\infty)]+E(\infty)
\end{gathered}
$$

[Since $E(\infty)=\gamma \phi(\infty)-i p A(\infty)$ ]

where the integration extends over all conducting sections. The equation must hold whether $(x y)$ is a point in a conductor or in a 
dielectric. Hence, after the value of $E$ has been determined for all points in conducting sections, this equation becomes an explicit formula giving the value of $E$ at any other point. As a matter of fact, the value of $E$ at outside points is seldom of practical interest, and the value of $E$ in conductors may be found without reference to its value in dielectrics. All of the conditions (8) except the last have been assumed in the derivation of (35) and (36), and it is easy to show from the properties of $M$ that if this equation is satisfied all of these conditions will be fulfilled with the exception of the last one, which requires that $r \frac{\partial E}{\partial r}$ (or $\left.r \frac{\partial A}{\partial r}\right)$ shall vanish canonically at infinity, a condition which is reciprocal with the relation $\sum_{1}^{N} I_{\mathrm{n}}=0$. This condition will, therefore, determine the value of $A(\infty)$ or $E(\infty)$. The integral equation (36) has a unique solution given in the form of a definite integral when the second member $F(x y)$ is any function given in all conducting sections which makes this integral convergent, so that the undetermined constant $E(\infty)$ which made its appearance in (36) must be so chosen as a linear function of the $N$ constants $c_{1}, c_{2}, \ldots c_{\mathrm{N}}$ that $\sum_{n=1}^{N} I_{\mathrm{n}}$ (where $I_{\mathrm{n}}$ is defined by (11) Section II) shall be zero whatever the values of $c_{1}, c_{3}, \ldots c_{\mathbb{N}}$. These constants may then all be considered as arbitrarily assignable. It may be noted that in case the $N^{\text {th }}$ conductor extends to infinity the value of $E(\infty)$ must be zero since $I_{\mathrm{N}}$ is assumed to be finite. Also, in this case $\phi_{\mathrm{N}}(\infty)=1$ and $\phi_{\mathrm{n}}(\infty)=0$ when $n \neq N$. Consequently, the second member of (36), namely $F(x y)$, which has different constant values on each conducting group, will have the value zero when $x y$ is on the $N^{\text {th }}$ conductor, since

$$
\gamma\left[\phi\left(x_{\mathrm{x}} y_{\mathrm{N}}\right)-\phi_{\mathrm{N}}(\infty)\right]+E(\infty)=\gamma[1-1]+0=0 .
$$

On any other conducting section $S_{\mathrm{n}}$ it has the value $\gamma c_{\mathrm{n}}$ if $n \neq N$.

\section{EXISTENCE AND UNIQUENESS OF A SOLUTION OF (36)}

Multi lying the expression (36)

$$
\left[E\left(x^{\prime} y^{\prime}\right)+i p \mathcal{S} \mathcal{S}\left(x^{\prime} y^{\prime} \xi \eta\right) \lambda(\xi \eta) E(\xi \eta) d \xi d \eta-F\left(x^{\prime} y^{\prime}\right)\right]=0
$$

by ip $M\left(x y x^{\prime} y^{\prime}\right) \lambda\left(x^{\prime} y^{\prime}\right) d x^{\prime} d y^{\prime}$ and integrating with respect of $x^{\prime} y^{\prime}$ over the entire range shows that if $E$ satisfies (36) it must also satisfy the equation

$$
\begin{gathered}
i p \mathcal{S} \mathcal{S} \lambda\left(x^{\prime} y^{\prime}\right) M\left(x y x^{\prime} y^{\prime}\right) d x^{\prime} d y^{\prime} . \\
{\left[E\left(x^{\prime} y^{\prime}\right)+i p \mathcal{S} \mathcal{S} M\left(x^{\prime} y^{\prime} \xi \eta\right) \lambda(\xi \eta) E(\xi \eta) d \xi d \eta-F\left(x^{\prime} y^{\prime}\right)\right]=0 .}
\end{gathered}
$$


Conversely, if this equation is satisfied, we may show that the bracket must be identically zero and, therefore, (36) will also be satisfied. To prove this let the bracket be represented by $h^{\prime}(x y)+i h^{\prime \prime}(x y)$. Then $h^{\prime}(x y)$ must be a real function such that

$$
\iint M\left(x y x^{\prime} y^{\prime}\right) h^{\prime}\left(x^{\prime} y^{\prime}\right) d x^{\prime} d y^{\prime}=0
$$

identically for all values of $x y$, and similarly for $h^{\prime \prime}$. Denoting this integral by $V(x y)$, it is evident that if $V(x y)=0$ identically then $\nabla^{2} V(x y)=0$ everywhere in the range. But $\nabla^{2} V(x y)=4 \pi \mu(x y) h(x y)$ as is seen by differentiation of the integral, and from the properties of $M$. Consequently, $h(x y)$ must be zero everywhere if $h$ is such that $\iint h^{2} d x d y$ exists. Therefore, the equations (36) and (37) are reciprocal.

By making use of (36) the equation (37) may be written

$$
\begin{gathered}
E(x y)+p^{2} \int \mathcal{S} d x^{\prime} d y^{\prime} M\left(x y x^{\prime} y^{\prime}\right) \lambda\left(x^{\prime} y^{\prime}\right) \int \mathcal{S} M\left(x^{\prime} y^{\prime} \xi \eta\right) \lambda(\xi \eta) E(\xi \eta) d \xi d \eta \\
=F(x y)-i p \int \mathcal{S} M(x y \xi \eta) \lambda(\xi \eta) F(\xi \eta) d \xi d \eta .
\end{gathered}
$$

The second member of this equation will be finite even if the $N^{\text {th }}$ conducting section extends to infinity, since $F(x y)$ will vanish on this section.

We may for the present limit the discussion to the case where all conducting sections are finite. The form thus obtained for the solution then suggests methods of dealing with the equation with open sections. With finite range the order of integration in the preceding integral may be interchanged and the equation takes the form

$$
\begin{aligned}
& E(x y)+p^{2} \int \mathcal{S} N(x y \xi \eta) \lambda(\xi \eta) E(\xi \eta) d \xi d \eta=f(x y) \\
& =F(x y)-i p \int \mathcal{S} M\left(x y x^{\prime} y^{\prime}\right) \lambda\left(x^{\prime} y^{\prime}\right) F\left(x^{\prime} y^{\prime}\right) d x^{\prime} d y^{\prime}
\end{aligned}
$$

where the new nucleus

$$
N(x y \xi \eta)=N(\xi \eta x y)=\iint M\left(x y x^{\prime} y^{\prime}\right) M\left(\xi \eta x^{\prime} y^{\prime}\right) \lambda\left(x^{\prime} y^{\prime}\right) d x^{\prime} d y^{\prime}
$$

is not only symmetrical in the two points, but is everywhere finitea property not possessed by $M$, which becomes logarithmically infinite when the two points approach each other. The theorems of Fredholm are applicable to this equation and show that if $p$ is not a root of the characteristic determinant, there is one and only one solution.

$$
\vec{E}(x y)=F(x y)-i p \int \mathcal{S} \mathfrak{N}(x y \xi \eta i p) F(\xi \eta) d \xi d \eta
$$

where $\mathfrak{R}(x y \xi \eta i p)=\frac{D_{\mathrm{N}}(x y \xi \eta i p)}{D_{\mathrm{N}}(i p)}$ is the resolving nucleus of the primitive $39058^{\circ}-25-4$ 
equation and is given by Fredholm's formula. It satisfies the two integral equations

$$
\begin{aligned}
& M\left(x y x^{\prime} y^{\prime}\right) \lambda\left(x^{\prime} y^{\prime}\right)-\mathfrak{R}\left(x y x^{\prime} y^{\prime} i p\right) \\
& =i p \mathcal{S} \mathcal{S} M(x y \xi \eta) \lambda(\xi \eta) \Re\left(\xi \eta x^{\prime} y^{\prime} i p\right) d \xi d \eta \\
& =i p \mathcal{S} \mathcal{S} M\left(\xi \eta x^{\prime} y^{\prime}\right) \lambda\left(x^{\prime} y^{\prime}\right) \Re(x y \xi \eta i p) d \xi d \eta .
\end{aligned}
$$

It is evident that $N(x y, x y)$ is finite since

$$
N(x y, x y)=\mathcal{S} \mathcal{S}\left[M\left(x^{\prime} y^{\prime} \xi \eta\right)\right]^{2} \lambda\left(x^{\prime} y^{\prime}\right) d x^{\prime} d y^{\prime}
$$

and the surface element when $x^{\prime} y^{\prime}$ is near the point $\xi \eta$ may be written in the form

$$
\begin{aligned}
\int_{0}^{2 \pi} d \theta \int_{0}^{\epsilon} r d r[-2 \mu \log r]^{2} & =8 \pi \mu^{2}(\xi \eta) \int_{0}^{\epsilon} r(\log r)^{2} d r \\
& =4 \pi \mu^{2}(\xi \eta) \epsilon^{2}\left[\log ^{2} \epsilon-\log \epsilon+\frac{1}{2}\right]
\end{aligned}
$$

and this vanishes with $\epsilon$, showing that the element of the surface integral near the point $\xi \eta$ contributes an infinitesimal amount to the integral.

If $p$ were a root of $D_{\mathrm{N}}(i p)=0$, then $\mathrm{N}$ would become infinite and the solution impossible in general. But it is shown that in this case there must be at least one solution $E_{\mathrm{o}}(x y)$, which is not identically zero, of the homogeneous equation obtained by placing the second member of (9) equal to zero. This solution $E_{\mathrm{o}}(x y)$ would also satisfy

$$
E_{0}(x y)+i p \mathcal{S} \mathcal{S} M(x y \xi \eta) \lambda(\xi \eta) E_{0}(\xi \eta) d \xi d_{\eta}=0 .
$$

If we write $\mathrm{E}_{0}(x y)=E^{\prime}{ }_{0}(x y)+i E^{\prime \prime}{ }_{0}(x y)$ where $E^{\prime}{ }_{0}$ and $E^{\prime \prime}{ }_{0}$ are real functions, and multiply this equation by

$$
\left[E^{\prime}{ }_{0}(x y)-i E^{\prime \prime}{ }_{0}(x y)\right] \lambda(x y) d x d y
$$

and integrate over the entire range, we obtain

$$
\begin{gathered}
\int \mathcal{S} \lambda(x y)\left[E^{\prime}{ }^{2}(x y)+E^{\prime \prime}{ }_{0}{ }^{2}(x y)\right] d x d y \\
+p \mathcal{S} \mathcal{S} d x d y \mathcal{S} \mathcal{S} d \xi d \eta M(x y \xi \eta) \lambda(x y) \lambda(\xi \eta)\left[E^{\prime}{ }_{0}(\xi \eta) E^{\prime \prime}{ }_{0}(x y)-E^{\prime}{ }_{0}(\xi \eta) E_{\mathrm{o}}\right. \\
(x y)]=-i p \mathcal{S} \mathcal{S} d x d y \mathcal{S} \mathcal{S} d \xi d \eta M(x y \xi \eta) \lambda(x y) \lambda(\xi \eta)\left[E^{\prime}{ }_{0}(\xi \eta) E^{\prime}{ }_{0}(x y)\right. \\
\left.+E^{\prime \prime}{ }_{0}(\xi \eta) E^{\prime \prime}{ }_{0}(x y)\right] .
\end{gathered}
$$

The second member of this equation is a pure imaginary, the first member real, hence each side must vanish. The second integral on the left vanishes because of the synmetrical property $M(x y \xi \eta)=M$ $(\xi \eta x y)$. Hence we must have

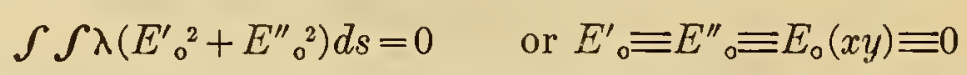

and, therefore, no real value of $p$ can be a characteristic constant for the primitive integral equation. A unique solution exists for all real values of $p$. 
4. OTHER METHODS OF SOLVING THE INTEGRAL EQUATION (36)

(a) By Normal Functions. - It is worth while to examine the equation from the point of view of normal functions. The nucleus $N\left(x y x^{\prime} y^{\prime}\right)$ is not only symmetrical and finite but it is definite, by which it is meant that there is no real function $h(x y)$ whose square is integrable (that is, such that $\int \mathcal{S} h^{2}(x y) d x d y$ exists and is not zero), which will make the integral $\int \mathcal{S} d x d y \int \mathcal{S} d x^{\prime} d y^{\prime} N\left(x y x^{\prime} y^{\prime}\right) h(x y) h\left(x^{\prime} y^{\prime}\right)$ vanish. This may be proven by noting that

$$
\begin{gathered}
\int \mathcal{S} d x d y \mathcal{S} \mathcal{S} d x^{\prime} d y^{\prime} N\left(x y x^{\prime} y^{\prime}\right) h(x y) h\left(x^{\prime} y^{\prime}\right) \\
=\mathcal{S} \mathcal{S} \lambda(\xi \eta) d \xi d \eta \mathcal{S} \mathcal{S} M(x y \xi \eta) h(x y) d x d y \mathcal{S} \mathcal{S} M\left(x^{\prime} y^{\prime} \xi \eta\right) h\left(x^{\prime} y^{\prime}\right) d x^{\prime} d y^{\prime} \\
=\mathcal{S} \mathcal{S} \lambda(\xi \eta) d \xi d \eta[\mathcal{S} \mathcal{S} M(x y \xi \eta) h(x y) d x d y]^{2}
\end{gathered}
$$

which is always $>0$ unless there is a function $\hbar$ for which $\mathcal{S} \mathcal{S} M(x y \xi \eta)$ $h(x y) d x d y=0$ identically for all points $\xi \eta$ in the range. We have just shown that no such function exists.

From this "definiteness" of $N$ it is known that its characteristic constants are infinite in number, real, and positive, and form a denumberable ensemble of isolated points. To each such constant $\tau_{\mathrm{n}}{ }^{2}$ there corresponds a, normal function $u_{\mathrm{n}}(x y)$ which is a funda- mental solution of the equation.

$$
u_{\mathrm{n}}(x y)-\tau_{\mathrm{n}}^{2} \mathcal{S} \mathcal{S} N(x y \xi \eta) u_{\mathrm{n}}(\xi \eta) d \xi d \eta=0
$$

The set of functions $u_{1}(x y), u_{2}(x y) \ldots \ldots$ are infinite in number and constitute a closed set of normal functions such that

$$
\begin{aligned}
\int \mathcal{S} u_{\mathbf{n}}(x y) u_{\mathrm{m}}(x y) d x d y & =0 \text { if } n \neq m \\
& =1 \text { if } n=m
\end{aligned}
$$

and the nucleus $N(x y \xi \eta)$ is equal to the uniformly convergent series

$$
N(x y \xi \eta)=\sum_{n=1}^{\infty} \frac{u_{n}(x y) u_{n}(\xi \eta)}{\tau^{2}{ }_{n}}
$$

An arbitrary function $f(x y)$ may be developed in a uniformly convergent series of these functions in the form

$$
f(x y)=\sum_{n=1}^{\infty} f_{\mathbf{n}} u_{\mathbf{n}}(x y)
$$

where the Fourier coefficient $f_{\mathrm{n}}$ is given by

$$
\left.f_{\mathrm{n}}=\int \mathcal{S} F(x y) u_{\mathrm{n}}(x y) d x d y\right)
$$

provided, the series $\sum_{1}^{\infty} f_{n}^{2}$ is convergent. Since the set of normal 
functions is "closed" there is no function which is normal to all of them; that is, orthogonal to $N(x y \xi \eta)$. The equation (39) may then be written

$$
\begin{gathered}
E(x y)+p^{2} \sum_{n=1}^{\infty} \frac{u_{\mathrm{n}}(x y)}{\tau^{2} \mathrm{n}} \iint_{\infty} u_{\mathrm{n}}(\xi \eta) \lambda(\xi \eta) E(\xi \eta) d \xi d \eta=f(x y) \\
=\sum_{n=1}^{\infty} f_{\mathrm{n}} u_{\mathrm{n}}(x y) .
\end{gathered}
$$

In the simplest case, where $\lambda$ has the same value in all materials, assume $E(x y)=\sum_{1}^{\infty} E_{\mathrm{n}} u_{\mathrm{n}}(x y)$ and substitute in (49). This gives

$$
\sum_{n=1}^{\infty} u_{\mathrm{n}}(x y)\left[E_{\mathrm{n}}+\frac{p^{2} \lambda}{\tau_{\mathrm{n}}^{2}} E_{\mathrm{n}}-f_{\mathrm{n}}\right]=0
$$

giving

and the solution is

$$
E_{\mathrm{n}}=\frac{f_{\mathrm{n}}}{1+\lambda \frac{p^{2}}{\tau^{2}}}
$$

$$
E(x y)=\sum_{n=1}^{\infty} \frac{F_{\mathrm{n}} u_{\mathrm{n}}(x y)}{1+\lambda \frac{p^{2}}{\tau^{2}{ }_{\mathrm{n}}}}=\iint F(\xi \eta) d \xi d \eta \sum_{n=1}^{\infty} \frac{u_{\mathrm{n}}(x y) u_{\mathrm{n}}(\xi \eta)}{1+\lambda \frac{p^{2}}{\tau^{2}{ }_{\mathrm{n}}}}
$$

In the general case, where $\lambda$ has different constant values in different conductors, if we write

and

$$
\lambda(x y) E(x y)=\sum_{n=1}^{\infty} b_{\mathrm{n}} u_{\mathrm{n}}(x y)
$$

$$
E(x y)=\sum_{n=1}^{\infty} E_{\mathrm{n}} u_{\mathrm{n}}(x y)
$$

and substitute in the equation, we find

$$
\sum_{n=1}^{\infty} u_{\mathrm{n}}(x y)\left[E_{\mathrm{n}}+\frac{p^{2}}{\tau^{2} \mathrm{n}} b_{\mathrm{n}}-f_{\mathrm{n}}\right]=0
$$

which will be satisfied if we may make

Now

$$
\frac{\tau^{2} \mathrm{n}}{p^{2}} E_{\mathrm{n}}+b_{\mathrm{n}}=\frac{\tau^{2}{ }_{\mathrm{n}} f_{\mathrm{n}}}{p^{2}} \quad \text { for } n=1,2,3 \cdots \cdots \infty \text {. }
$$

$$
\begin{aligned}
b_{\mathrm{n}} & =\iint u_{\mathrm{n}}(x y) \lambda(x y) E(x y) d x d y \\
& =\iint u_{\mathrm{n}}(x y) \lambda(x y) \sum_{\mathrm{k}=1}^{\infty} E_{\mathbf{k}} u_{\mathrm{k}}(x y) d x d y
\end{aligned}
$$

or

where

$$
b_{\mathrm{n}}=\sum_{\mathrm{k}=1}^{\infty} g_{\mathrm{nk}} E_{\mathrm{k}}
$$

$$
g_{\mathrm{nk}}=g_{\mathrm{kn}}=\iint \lambda(x y) u_{\mathrm{n}}(x y) u_{\mathrm{k}}(x y) d x d y \text {. }
$$


Hence, the constants $E_{\mathbf{n}}$ will be the solution of an infinite set of linear equations

or

$$
\frac{\tau^{2}}{p_{2}} E_{\mathrm{n}}+\sum_{\mathrm{k}=1}^{\infty} g_{\mathrm{nk}} E_{\mathrm{k}}=\frac{\tau^{2} f_{\mathrm{n}}}{p_{2}} \quad \text { for } n=1,2,3 \cdots \cdots N
$$

$$
\begin{aligned}
& \left(\frac{\tau_{1}^{2}}{p^{2}}+g_{11}\right) E_{1}+g_{12} E_{2}+g_{13} E_{3} \ldots \ldots \ldots .=\frac{\tau_{1}^{2} f_{1}}{p^{2}} \\
& g_{21} E_{1}+\left(\frac{\tau_{2}^{2}}{p^{2}}+g_{22}\right) E_{2}+g_{23} E_{3} \ldots \ldots \ldots .=\frac{\tau_{2}^{2} f_{2}}{p^{2}} \\
& g_{31} E_{1}+g_{32} E_{2}+\left(\frac{\tau^{2}}{p^{2}}+g_{33}\right) E_{3} \ldots \ldots \ldots=\frac{\tau_{3}^{2} f_{3}}{p^{2}}
\end{aligned}
$$

This set may be solved because its determinant can not vanish for any real value of $p$.

In case $\lambda$ has the same constant value on all conductors

$$
\begin{aligned}
& g_{\mathrm{nk}}=0 \text { if } n \neq k \\
& g_{\mathrm{nn}}=\lambda
\end{aligned}
$$

and we obtain the previous formula.

In the case where a conductor extends to infinity, the range of integration is no longer finite and the characteristic values of a finite symmetrical nucleus would no longer constitute a set of isolated points, but would become uniformly distributed along the real axis everywhere equally dense. The representation of an arbitrary function in an infinite series of normal functions, over a definite range, would then give place to its representation over the infinite range by a definite integral of which Fourier's integral is an example. Instead of a solution in an infinite series of normal functions, one may expect a solution in the form of a definite integral.

(b) Method of Successive Integration at Low FrequencIEs.-It may be noted that the method of iterated integrations, which is always applicable to Volterra's type of equation, may be successfully applied to the present problem if the frequency is small. This gives the solution in ascending powers of $i p$ and is only applicable for values of $p$ less than the modulus of the first characteristic constant of the primitive equation. The solution will be identical with that obtained from the Maclaurin development of

$$
\Re(x y \xi \eta i p)=\frac{D_{\mathrm{N}}(x y \xi \eta i p)}{D_{\mathrm{N}}(i p)}
$$

in ascending powers of $p$. Although no real value of $p$ can be a root of $D_{\mathrm{N}}(i p)=0$, nevertheless the Maclaurin development is limited to the circle in the complex $p$ plane, whose radius is less than the modulus of the smallest complex root. 
(c) Method of Harmonic Analysis.-It seems probable that in the majority of cases there will be less labor involved in solving the differential equation with its boundary conditions by some sort of series of harmonic functions, such as Fourier-Bessel expansions, than in solving the integral equation for $A$ (or $E$ ).

The differential equation for $A$ suggests certain types of expansions for $A$ as a series of appropriate functions in each conductor or group of conductors which will be normal functions for that section. If there are groups of conductors in contact for which $\lambda$ or $\mu$ have different values, the continuity of $A$ and of $\frac{1}{\mu} \frac{\partial A}{\partial n}$ at such internal boundaries will lead to certain relations between the coefficients. The mutual influence which the conducting groups exert upon each other across the intervening dielectrics may then be found by assuming certain forms of expansion for $A$ in the dielectric and by then making $A$ and $\frac{1}{\mu} \frac{\partial A}{\partial n}$ continuous at every boundary between conductors and dielectric. This step may, however, be replaced by the following process which avoids any reference to the dielectric.

(d) Mrxed Method.-Let $x y$ be a point within any conducting section, and with $\xi \eta$ as the variable of integration apply Green's theorem to the two functions $A(\xi \eta)$ and $\log r(x y \xi \eta)$ for the entire dielectric region. This gives

$$
A(\infty)+\frac{1}{2 \pi} \int\left[A(\xi \eta) \frac{\partial \log r(x y \xi \eta)}{\partial n}-\log r(x y \xi \eta) \frac{\partial A(\xi \eta)}{\partial n}\right] d s=0
$$

where the integration is taken in the dielectric just outside the conductors and around the complete boundary of all conductors. Since $A$ and $\frac{1}{\mu} \frac{\partial A}{\partial n}$ are continuous at such boundaries, this necessary condition which $A$ must satisfy becomes

$$
A(\infty)=\frac{1}{2 \pi} \int\left[A(\xi \eta) \frac{\partial \log r(x y \xi \eta)}{\partial n_{\mathbf{i}}}-\log r(x y \xi \eta) \frac{1}{\mu} \frac{\partial A}{\partial n_{\mathbf{i}}}\right] d s
$$

where the integration is taken around the complete boundary of all conducting groups as before but just inside the conductors. The internal normal $n_{\mathbf{i}}$ points toward the boundary. If the series for the internal values of $A$ at each conducting group be introduced in to this integral, the result must be identically true in whatever conducting section the point $x y$ may be. By expressing this fact, when $x y$ is in each group in succession, the required number of equations between the coefficients are obtained. This method is illustrated in the case of two circular cylinders at the end of this paper. 


\section{FORM AND PROPERTIES OF THE SOLUTION}

The $n$ functions $\omega_{1}(x y), \omega_{2}(x y), \cdots \cdots \omega_{\mathbb{N}}(x y)$, which are defined as the solutions of integral equations of type

$$
\omega_{\mathbf{k}}(x y)+i p \mathcal{S} \mathcal{S} M(x y \xi \eta) \lambda(\xi \eta) \omega_{\mathbf{k}}(\xi \eta) d \xi d \eta=\phi_{\mathbf{k}}(x y)
$$

are all linearly independent, for if there were a relation of the form $\sum_{k=1}^{N} h_{\mathrm{k}} \omega_{\mathrm{k}}(x y)=0$ the above equation, on being multiplied by $h_{\mathrm{k}}$, and the sum taken for all values of $k$ leads to

$$
\sum_{k=1}^{N} h_{\mathrm{k}} \omega_{\mathrm{k}}(x y)+i p \mathcal{S} \mathcal{S} M(x y \xi \eta) \lambda(\xi \eta) \sum_{k=1}^{N} h_{\mathrm{k}} \omega_{\mathrm{k}}(\xi \eta) d \xi d_{\eta}=\sum_{k=1}^{N} h_{\mathrm{k}} \phi_{\mathrm{k}}(x y) .
$$

The hypothesis leads to the conclusion that $\sum_{k=1}^{N} h_{\mathbf{k}} \phi_{\mathrm{k}}(x y)=0$ for all values of $x y$ in the range. But since $\phi_{\mathrm{r}}(x y)=1$ when $x y$ is on conducting section $S_{\mathrm{k}}$ and equals 0 when $x y$ is on $S_{\mathrm{n}}$ where $n \neq k$, it follows that $h_{1}=h_{2} \cdots \cdots=h_{\mathrm{n}}=0$.

It is also evident that the only solution of

is

$$
\omega(x y)+i p \mathcal{S} \mathcal{S} M(x y \xi \eta) \lambda(\xi \eta) \omega(\xi \eta) d \xi d \eta=1
$$

$$
\omega(x y)=\sum_{k=1}^{N} \omega_{\mathrm{k}}(x y) \text {. }
$$

The solution for $E$ is therefore

and

$$
E(x y)=\sum_{k=1}^{N}\left[\gamma c_{\mathrm{k}}-i p A(\infty)\right] \omega_{\mathrm{k}}(x y)
$$

where

$$
I_{\mathrm{n}}=\sum_{k=1}^{N}\left[\gamma c_{\mathrm{k}}-i p A(\infty) b_{\mathrm{nk}}\right]
$$

$$
b_{\mathrm{nk}}=\int \mathcal{S} \lambda \omega_{\mathrm{k}} d S_{\mathrm{n}}
$$

the integration being taken over the section $S_{\mathrm{n}}$ of the $n_{\text {th }}$ conducting group. The array of complex constants $b_{\mathrm{nk}}$ is symmetrical; that is,

$$
b_{\mathrm{nk}}=b_{\mathrm{kn}} \text {. }
$$

To show this, multiply the equation for $\omega_{\mathrm{k}}$ by $\lambda(x y) \omega_{\mathrm{n}}(x y) d x d y$ and integrate over the entire range. This gives

$$
\begin{aligned}
& \int \mathcal{S} \lambda(x y) \omega_{\mathbf{n}}(x y) \omega_{\mathbf{k}}(x y) d x d y \\
+ & i p \mathcal{S} \mathcal{S} d x d y \mathcal{S} \mathcal{S} d \xi d \eta \dot{M}(x y \xi \eta) \lambda(x y) \omega_{\mathbf{n}}(x y) \lambda(\xi \eta) \omega_{\mathbf{k}}(\xi \eta) \\
= & \mathcal{S} \mathcal{S} \lambda(x y) \omega_{\mathbf{n}}(x y) \phi_{\mathbf{k}}(x y) d x d y=\int \mathcal{S} \lambda \omega_{\mathbf{n}} d S_{\mathbf{k}}=b_{\mathbf{k n}} .
\end{aligned}
$$


If we multiply the equation for $\omega_{\mathrm{n}}$ by $\lambda \omega_{\mathrm{k}} d x d y$ and integrate, the right-hand side is $b_{\mathrm{nk}}$ and the left side is easily seen to be identical with the above on account of the symmetrical property $M(x y \xi \eta)=M(\xi \eta x y)$.

The constant $A(\infty)$ must be so chosen that the sum of all the currents $I_{\mathrm{n}}$ will vanish. If the expression (53) for $E$ be multiplied by $\lambda(x y) d x d y$ and the integration taken over all conductors, this gives

or

$$
\begin{gathered}
\iint \lambda E d x d y=\sum_{n=1}^{N} I_{\mathrm{n}}=0=\gamma \sum_{k=1}^{N} c_{\mathbf{k}} \iint \lambda(x y) \omega_{\mathbf{k}}(x y) d x y \\
-i p A(\infty) \iint \lambda \sum_{k=1}^{N} \omega_{\mathbf{K}}(x y) d x d y
\end{gathered}
$$

where

$$
i p A(\infty)=-E(\infty)+\gamma \phi(\infty)=\frac{\gamma \sum_{n=1}^{N} \sum_{k=1}^{N} b_{\mathrm{nk}} c_{\mathrm{k}}}{\sum_{n=1}^{N} \sum_{k=1}^{N} b_{\mathrm{nk}}}=\gamma \sum_{k=1}^{N} \Psi_{\mathrm{k}}(\infty) c_{\mathrm{k}}
$$

which shows that

$$
\Psi_{\mathrm{k}}(\infty) \equiv \frac{\sum_{n=1}^{N} b_{\mathrm{nk}}}{\sum_{n=1}^{N} \sum_{s=1}^{N} b_{\mathrm{ns}}}
$$

$$
\sum_{k=1}^{N} \Psi_{\mathrm{k}}(\infty)=1
$$

In order that it shall always be possible to thus choose the constant $A(\infty)$ as in (57), so as to make $\sum I_{n}=0$, the denominator in the above formula for $\Psi_{\mathrm{k}}(\infty)$, namely,

$$
\sum_{n=1}^{N} \sum_{k=1}^{N} b_{\text {nk }} \text { or } \iint \lambda \sum \omega_{\mathrm{k}}(x y) d x d y
$$

must nerer be zero. To prore that this can nerer vanish let

$$
\omega(x y)=\sum_{k=1}^{N} \omega_{K}(x y)=u(x y)+i v(x y)
$$

where $u$ and $v$ are real functions satisfying

$$
\begin{aligned}
& u(x y)-p \mathcal{S} \mathcal{S} M(x y \xi \eta) \lambda(\xi \eta) v(\xi \eta) d \xi d \eta=1 \\
& v(x y)+p \mathcal{S} \mathcal{S} M(x y \xi \eta) \lambda(\xi \eta) u(\xi \eta) d \xi d \eta=0 .
\end{aligned}
$$

Multiply the first of these by $\lambda(x y) u(x y) d x d y$, the second by $\lambda(x y) v(x y) d x d y$, add the results, and integrate orer the range. This gires on account of the symmetry of $M$

$$
\int \mathcal{S} \lambda(x y)\left[u^{2}(x y)+v^{2}(x y)\right] d x d y=\int \mathcal{S} \lambda(x y) u(x y) d x d y
$$

Now, if the sum in question were zero, then

$$
\int \mathcal{S} \lambda u d x d y=\int \mathcal{S} \lambda \tau d x d y=0
$$


and in this case the above relation would require that $u$ and $v$ and hence

$$
\omega \equiv \sum_{k=1}^{N} \omega_{\mathrm{k}}(x y)
$$

be identically zero at all points of the range, which is never the case since the functions $\omega_{\mathrm{k}}(x y)$ are not linearly dependent. This shows not only that the double sum in the denominator of (58) never vanishes, but that its real part is always positive. Consequently, it will always be possible to choose $A(\infty)$ by (57) and (58), so that the sum of the currents will vanish. A similar treatment of $\omega_{\mathrm{k}}(x y)$ shows that the real part of $b_{\mathrm{kk}}$ is positive and less than $\frac{1}{R_{\mathrm{k}}(0)}$.

Inserting the value of $i p A(\infty)$ in the expression (53) for $E(x y)$ gives

$$
\begin{aligned}
E(x y) & =\gamma \sum_{k=1}^{N} c_{\mathrm{k}} \omega_{\mathrm{k}}(x y)-\gamma \sum_{s=1}^{N} \omega_{\mathrm{s}}(x y) \sum_{k=1}^{N} \Psi_{\mathrm{k}}(\infty) c_{\mathrm{k}} \\
& =\gamma \sum_{k=1}^{N} c_{\mathrm{k}}\left[\omega_{\mathrm{k}}(x y)-\Psi_{\mathrm{k}}(\infty) \sum_{s=1}^{N} \omega_{\mathrm{s}}(x y)\right] .
\end{aligned}
$$

If we define the new set of functions $\Omega_{1}, \Omega_{2}, \ldots \Omega_{\mathrm{n}} \Omega_{\mathrm{N}}$ by

$$
\Omega_{\mathrm{k}}(x y) \equiv \omega_{\mathrm{k}}(x y)-\Psi_{\mathrm{k}}(\infty) \sum_{s=1}^{N} \omega_{\mathrm{s}}(x y), k=1,2, \cdots N
$$

then

$$
E(x y)=\gamma \sum_{k=1}^{N} c_{\mathrm{k}} \Omega_{\mathrm{k}}(x y)
$$

From this definition it follows that

$$
\int \mathcal{S} \lambda(x y) \Omega_{\mathrm{k}}(x y) d x d y=0, k=1,2,3 \cdots N .
$$

Substituting

$$
\omega_{\mathrm{k}}(x y)=\Omega_{\mathrm{k}}(x y)+\Psi_{\mathrm{k}}(\infty) \sum_{s=1}^{N} \omega_{\mathrm{s}}(x y)
$$

in the integral equation (52) for $\omega_{\mathrm{k}}$ gives

$$
\begin{aligned}
\Omega_{\mathrm{k}}(x y) & +i p \mathcal{S} \mathcal{S} M(x y \xi \eta) \lambda(\xi \eta) \Omega_{\mathrm{k}}(\xi \eta) d \xi d \eta+\Psi_{\mathrm{k}}(\infty) \sum_{s=1}^{N} \omega_{\mathrm{s}}(x y) \\
& \left.+i p \mathcal{S} \mathcal{S} M(x y \xi \eta) \lambda(\xi \eta) \omega_{\mathrm{s}}(\xi \eta) d \xi d \eta\right]=\phi_{\mathrm{k}}(x y) .
\end{aligned}
$$

The bracket in the equation is just $\phi_{\mathrm{s}}(x y)$ and $\sum_{s=1}^{N} \phi_{\mathrm{s}}(x y)=1$.

Hence, $\Omega_{\mathrm{k}}(x y)$ is the solution of the integral equation

$$
\Omega_{\mathrm{k}}(x y)+i p \mathcal{S} \mathcal{S} M(x y \xi \eta) \lambda(\xi \eta) \Omega_{\mathrm{k}}(\xi \eta) d \xi d \eta=\phi_{\mathrm{k}}(x y)-\Psi_{\mathrm{k}}(\infty) .
$$


This equation suffices to uniquely determine each function $\Omega_{\mathrm{k}}(x y)$ at all points in conductors. However, the $N$ functions thus determined will not all be independent for since

$$
\sum_{k=1}^{N} \Psi_{\mathbf{k}}(\infty)=1
$$

it follows from the definition of $\Omega_{\mathrm{k}}$ that

$$
\sum_{k=1}^{N} \Omega_{\mathrm{k}}(x y)=0 \text {. }
$$

If we define the coefficient $\beta_{\mathrm{n} \text { r }}$ by

$$
\beta_{\mathrm{nk}} \equiv \mathcal{S} \lambda \lambda \Omega_{\mathrm{k}} d S_{\mathrm{n}} .
$$

Then the array of coefficients $\beta_{\mathrm{n} \text { k }}$ is symmetrical.

$$
\beta_{\mathrm{nk}}=\beta_{\mathrm{kn}}
$$

This is proven in a manner precisely similar to that used in proving $b_{\mathrm{nk}}=b_{\mathrm{kn}}$. The constants $\beta_{\mathrm{nk}}$ also possess the property corresponding to equation (31)

$$
\sum_{n=1}^{N} \beta_{\mathrm{nk}}=0 \quad k=1,2,3 \ldots \ldots N .
$$

The equation of definition of current for each conducting group takes the form

$$
I_{\mathrm{n}}=\gamma \sum_{k=1}^{N} \beta_{\mathrm{nk}} c_{\mathrm{k}}=\gamma \sum_{\mathrm{k}=1}^{N} \beta_{\mathrm{nk}}\left(c_{\mathrm{k}}-c_{\mathrm{N}}\right) \text { for } n=1,2,3 \ldots N .
$$

From some points of view it is more simple to introduce in place of the function $\Omega_{\mathrm{z}}$ the functions $\Psi_{1}(x y) \ldots \ldots \Psi_{\mathrm{n}}(x y)$ defined by

$$
\Psi_{\mathrm{k}}(x y)=\phi_{\mathrm{k}}(x y)-\Omega_{\mathrm{k}}(x y) .
$$

Each must be the solution of an integral equation of the type

$$
\Psi_{\mathrm{k}}(x y)+i p \mathcal{S} \mathcal{S} M(x y \xi \eta) \lambda(\xi \eta)\left[\Psi_{\mathrm{k}}(\xi \eta)-\phi_{\mathrm{k}}(\xi \eta)\right] d \xi d \eta=\Psi_{\mathrm{k}}(\infty) .
$$

This equation suffices to uniquely determine $\Psi(x y)$ at all points in conductors and serves as a definition of $\Psi_{\mathrm{k}}(x y)$ when the point $x y$ is in a dielectric. The vector potential $A(x y)$ is given at any point $x y$ by

$$
A(x y)=\frac{\gamma}{i p} \sum_{k=1}^{N} c_{\mathrm{k}} \Psi_{\mathrm{k}}(x y)
$$

which presents a certain formal analogy to the expression for the scalar potential

$$
\phi(x y)=\sum_{k=1}^{N} c_{\mathrm{k}} \phi_{\mathrm{k}}(x y) .
$$


The set of functions $\Psi_{\mathbf{k}}(x y)$ and $\phi_{\mathbf{k}}(x y)$ present a close analogy, for the symmetrical set of complex coefficients $\beta_{\mathrm{nk}}$ are analogous to the set $\alpha_{n k}$. In fact, the previous definition of $\beta_{n k}$ may be reduced to a form precisely similar to that of $\alpha_{\mathrm{nk}}$, namely,

$$
\beta_{\mathrm{n} \mathbf{k}}=\frac{1}{4 \pi i p} \int \frac{\partial \Psi_{\mathrm{k}}}{\partial n} d s_{\mathrm{n}}
$$

where the integration is taken around any closed curve in the dielectric region which encircles the $n^{\text {th }}$ conducting group only, and where the normal points toward this conduction. This is evident since $\Psi_{\mathrm{k}}$ is harmonic in the dielectric, from its definition. Also since

$$
\mathcal{S} \int \lambda \Omega_{\mathrm{k}} d x d y=\sum_{n=1}^{N} \mathcal{S} \mathcal{S} \lambda \Omega_{\mathrm{k}} d S_{\mathrm{n}}=\mathcal{S} \mathcal{S} \lambda \Psi_{\mathrm{k}} d x d y=\sum_{n=1}^{N} \beta_{\mathrm{nk}}=0
$$

it follows that $\Psi_{\mathbf{k}}(x y)$ approaches the finite constant value $\Psi_{\mathbf{k}}(\infty)$ when the point $x y$ moves off to infinity. The $\operatorname{limit}_{r=\infty} r \frac{\partial \Psi_{k}}{\partial r}=0$. By differentiating the integral it is seen that

$$
\begin{aligned}
\nabla^{2} \Psi_{\mathbf{k}}(x y) & =0 \text { if } x y \text { is in a dielectric } \\
& =4 \pi i p \mu \lambda \Psi_{\mathbf{k}}(x y) \text { if } x y \text { is in a conductor section } \\
& \mathrm{S}_{\mathrm{n}} \text { and } n \neq k \\
& =4 \pi i p \mu \lambda\left[\Psi_{\mathrm{k}}(x y)-1\right] \text { if } n=k
\end{aligned}
$$

The analogy to $\sum_{k=1}^{N} \phi_{\mathrm{k}}(x y)=1$ is the relation

$$
\sum_{k=1}^{N} \Psi_{k}(x y)=1
$$

It is also evident from the properties of $M$ that $\Psi_{\mathrm{k}}(x y)$ and $\frac{1}{\mu} \frac{\partial \Psi_{\mathrm{k}}}{\partial n}$ are everywhere continuous. The integral equation for $\Psi_{\mathrm{k}}$ will determine its value in conductors without reference to the dielectric. However, it may be more convenient in practice to determine these functions by the methods of harmonic analysis. The foregoing differential equations and boundary conditions suffice to uniquely determine them at all points. Only $N-1$ of them need be computed, the remaining one being given by (73), which holds at all points. The condition that $\operatorname{limit}_{r=\infty}\left(r \frac{\partial \Psi_{\mathrm{k}}}{\partial r}\right)=0$ will (together with the other conditions) determine the value of $\Psi_{\mathrm{k}}(\infty)$.

It is evident from this point of view that the case where a conductor extends to infinity presents no exception to the general form of solution or the properties of these functions. The foregoing method of determining the $N$ function of $\Psi_{\mathrm{k}}$, only $N-1$ of which are independent, amounts to a determination of the constant of integration $A(\infty)$ at the outset so as to satisfy the condition $\sum_{n=1}^{N} I_{n}=0$ for all values of $\gamma, c_{1}, c_{2}, \ldots \ldots c_{\mathbb{N}}$. 
The following alternative procedure amounts to an elimination of this constant $A(\infty)$ and leads immediately to a value of $\gamma$ and the reactance of the system; in fact, to all information of practical value. While not as symmetrical as the first method, it is essentially the same and possesses the advantage of leading naturally to a notation which reduces to a familiar one when the conductors are linear or the frequency low. It consists in finding the $N$ independent functions $\omega_{1}(x y) \omega_{2}(x y) \ldots \ldots \omega_{N}(x y)$, or what is the same thing, the $N$ independent functions which will be donated by the small letters $\psi_{1}(x y) \psi_{2}(x y) \ldots \psi_{\mathrm{N}}(x y)$ and defined by

$$
\psi_{\mathrm{k}}(x y)=\phi_{\mathrm{k}}(x y)-\omega_{\mathrm{k}}(x y)
$$

The integral equation for $\omega_{\mathrm{k}}(x y)$ shows that $\psi_{\mathrm{k}}(x y)$ is the solution of the integral equation

$$
\begin{aligned}
\psi_{\mathrm{k}}(x y) & +i p \mathcal{S} \mathcal{S} M(x y \xi \eta) \lambda(\xi \eta) \psi_{\mathbf{k}}(\xi \eta) d \xi d \eta \\
& =i p \mathcal{S} \mathcal{S} M(x y \xi \eta) \lambda(\xi \eta) \phi_{\mathbf{k}}(\xi \eta) d \xi d \eta
\end{aligned}
$$

This serves to uniquely determine $\psi_{\mathrm{k}}(x y)$ at all points in conductors and thence to define its value at all dielectric points. For the practical evaluation of $\psi_{\mathrm{k}}(x y)$ by harmonic analysis, as the solution of a differential equation with certain boundary conditions, we may derive the following equations and conditions which $\psi_{\mathrm{k}}(x y)$ must satisfy from a consideration of this integral equation.

By differentiation under the integral sign one finds from the properties of $M$ that

(a) $\nabla^{2} \psi_{\mathrm{k}}(x y)=0$ if $x y$ is in a dielectric

$$
=4 \pi i p \mu \lambda\left[\psi_{\mathrm{k}}(x y)-\phi_{\mathrm{k}}(x y)\right] \text { if in a conductor }
$$

(b) $\psi_{\mathrm{k}}$ and $\frac{1}{\mu} \frac{\partial \psi_{\mathrm{k}}}{\partial n}$ are continuous

(c) $\quad \psi_{\mathrm{k}}(x y)=D_{\mathrm{k}} \log r(00 x y)$ at infinity, where $D_{\mathrm{k}}$ is a determinate constant not assignable.

These conditions are easily seen to be reciprocal with the integral equation, which may, in fact, be derived from them just as the equation for $A$ and $E$ was derived. Since

$$
E(x y)=\gamma \phi(x y)-i p A(x y)=\gamma \sum_{\mathrm{k}=1}^{\mathrm{N}} c_{\mathrm{k}} \phi_{\mathrm{k}}(x y)-i p A(x y)
$$

the vector potential $A(x y)$ at any point in the plane will be given by

and

$$
i p A(x y)=i p A(\infty)+\sum_{\mathrm{k}=1}^{\mathrm{N}}\left[\gamma c_{\mathrm{k}}-i p A(\infty)\right] \psi_{\mathrm{k}}(x y)
$$

$$
\Lambda_{\mathrm{n}}=\sum_{\mathrm{k}=1}^{\mathrm{N}}\left[\gamma c_{\mathrm{k}}-i p A(\infty)\right] b_{\mathrm{nk}}
$$


The definition of $b_{\mathrm{nk}}$ already given is easily seen to be equivalent to

$$
b_{\mathrm{nk}}=\frac{1}{4 \pi i p} \int \frac{\partial \psi_{\mathrm{k}}}{\partial_{\mathrm{n}}} d s_{\mathrm{n}}
$$

taken around any closed curve in the dielectric which encircles the $n^{\text {th }}$ group of conductors only.

\section{COEFFICIENTS OF RESISTANCE AND INDUCTANCE}

If it can be shown that the determinant $\left|b_{n k}\right|$ formed with the array of constants $b_{\mathrm{nk}}$ is never zero, then the equations (78) may be solved giving the forms

where

$$
\gamma c_{\mathrm{s}}-i p A(\infty)=\sum_{\mathrm{k}=1}^{\mathrm{N}} z_{\mathrm{sk}} I_{\mathrm{k}} \cdots \text { for } s=1,2,3 \cdots \cdots N
$$

$$
z_{\mathrm{sk}}=R_{\mathrm{sk}}+i p L_{\mathrm{sk}}
$$

and the real constants $R_{\mathrm{sk}}$ and $L_{\mathrm{sk}}$ are coefficients of resistance and inductance, respectively. Since they are derived from a system of equations with a symmetrical determinant, the coefficients $z_{n k}$ are symmetrical.

$$
z_{\mathrm{nk}}=z_{\mathrm{kn}} \text { or } \begin{gathered}
R_{\mathrm{nk}}=R_{\mathrm{kn}} \\
L_{\mathrm{nk}}=L_{\mathrm{kn}}
\end{gathered}
$$

The determinant $\left|b_{\mathbf{n} \mathbf{k}}\right|$ can not vanish for any finite value of $p$ with finite conductivities. For we have shown that it is always possible to so choose the constant $A(\infty)$, without making each current $I_{n}$ vanish, that the sum of the currents $\sum_{n=1}^{N} I_{n}$ shall be zero for every possible assignment of values to the $N$ constants $c_{1}, c_{2}, \cdots c_{\mathrm{N}}$, and a direct contradiction to this fact may be obtained by assuming the determinant $\left|b_{\mathrm{nk}}\right|$ to vanish. For in that event, it would be possible to choose the constants $c_{1}, c_{2}, \cdots c_{\mathbb{N}}$ not all zero, such that

$$
\sum_{k=1}^{N} b_{\mathrm{nk}} c_{\mathrm{k}}=0 \text { for } n=1,2,3 \cdots \cdots N
$$

in which case each current $I_{\mathrm{n}}$ would be given by

and if

$$
I_{\mathrm{n}}=-i p A(\infty) \sum_{k=1}^{N} b_{\mathrm{nk}}
$$

$$
\sum_{n=1}^{N} I_{\mathrm{n}}=0=-i p A(\infty) \sum_{n=1}^{N} \sum_{k=1}^{N} b_{\mathrm{nk}}
$$

this will require that $A(\infty)=0$, since it has been shown that the double sum can never vanish. Consequently, this would require that each current $I_{1}=I_{2} \cdots=I_{\mathrm{N}}=0$. 
Therefore, the equations (78) may always be solved and the coefficients $R_{\mathrm{nk}}$ and $L_{\mathrm{nk}}$ found. This being done, it will appear that the value of $\gamma$ and the reactance of the line may be found without the necessity of computing the value of $A(\infty)$.

In case the frequency is very small, the function $\omega_{\mathbf{k}}(x y)$ is almost identical with $\phi_{\mathrm{k}}(x y)$ so that $b_{\mathrm{nk}}$ approaches zero with vanishing frequency if $n \neq k$, and $b_{\mathrm{kk}}$ approaches $\frac{1}{R_{\mathrm{k}}(0)}$ where $R_{\mathrm{k}}(0)$ is the direct current resistance per unit length of the $k^{\text {th }}$ conducting group. The determinant $\left|b_{\mathrm{nk}}\right|$ approaches

$$
\left|\begin{array}{ccccc}
\frac{1}{R_{1}(0)} & 0 & 0 & \cdots \cdots & 0 \\
0 & \frac{1}{R_{2}(0)} & 0 & \cdots \cdots & 0 \\
0 & 0 & - & \cdots \cdots & 0 \\
\cdots \cdots & \ldots \ldots & \ldots & \ldots & \ldots \\
0 & 0 & 0 & \cdots \cdots & \frac{1}{R_{\mathrm{N}}(0)}
\end{array}\right|
$$

The current distribution is practically uniform over the section of each conductor and $R_{\mathrm{nn}}$ approaches $R_{\mathrm{n}}(0)$ while $R_{\mathrm{nk}}$ becomes negligible if $n \neq k$ with vanishing frequency.

This approximation neglects $\psi_{\mathbf{k}}$ because of the smallness of $p$, but the next approximation gives

which gives

$$
\psi_{\mathrm{k}}(x y)=i p \mathcal{S} \mathcal{S} M\left(x y \xi_{\mathrm{k}} \eta_{\mathrm{k}}\right) \lambda\left(\xi_{\mathrm{k}} \eta_{\mathrm{k}}\right) d S_{\mathrm{k}}
$$

$$
\begin{aligned}
b_{\mathrm{n} \mathbf{k}} & =-i p \mathcal{S} \mathcal{S} d S_{\mathrm{n}} \mathcal{S} \mathcal{S} d S_{\mathrm{k}} \lambda\left(x_{\mathrm{n}} y_{\mathrm{n}}\right) M\left(x_{\mathrm{n}} y_{\mathrm{n}} \xi_{\mathrm{k}} \eta_{\mathrm{k}}\right) \lambda\left(\xi_{\mathrm{k}} \eta_{\mathrm{k}}\right) \\
& =-i p \frac{L_{\mathrm{nk}}}{R_{\mathrm{n}}(0) R_{\mathrm{k}}(0)} \text { if } n \neq k .
\end{aligned}
$$

And when $n=k$

$$
\begin{aligned}
b_{\mathrm{nn}} & =\frac{1}{R_{\mathrm{n}}(0)}-i p \mathcal{S} \mathcal{S} d S_{\mathrm{n}} \mathcal{S} \mathcal{S} d S_{\mathrm{k}} \lambda\left(x_{\mathrm{n}} y_{\mathrm{n}}\right) M\left(x_{\mathrm{n}} y_{\mathrm{n}} \xi_{\mathrm{n}} \eta_{\mathrm{n}}\right) \lambda\left(\xi_{\mathrm{n}} \eta_{\mathrm{n}}\right) \\
& =\frac{1}{R_{\mathrm{n}}(0)}-i p \frac{L_{\mathrm{nn}}}{R_{\mathrm{n}}(0)^{2}} .
\end{aligned}
$$

The equations of definition of current (78) become

$$
\begin{gathered}
I_{\mathrm{n}}=\frac{\lambda c_{\mathrm{n}}-i p A(\infty)}{R_{\mathrm{n}}(0)}-i p \sum_{k=1}^{N} \frac{\lambda c_{\mathrm{k}}-i p A(\infty) L_{\mathrm{nk}}}{R_{\mathrm{k}}(0) R_{\mathrm{n}}(0)} \\
\quad \text { for } n=1,2,3 \ldots \ldots \cdots N .
\end{gathered}
$$


The first approximation to a solution is

$$
\frac{\gamma c_{\mathrm{k}}-i p A(\infty)}{R_{\mathrm{k}}(0)}=I_{\mathrm{k}}
$$

and placing this value in the above summation gives

$$
\gamma c_{\mathrm{n}}-i p A(\infty)=R_{\mathrm{n}}(0) I_{\mathrm{n}}+i p \sum_{k=1}^{N} L_{\mathrm{nk}} I_{\mathrm{k}}
$$

which shows that for low frequencies $R_{\mathrm{nn}}=R_{\mathrm{n}}(0)$ and $R_{\mathrm{nk}}=0, n \neq k$ while

$$
\begin{aligned}
& L_{\mathrm{nk}}=L_{\mathrm{kn}}=R_{\mathrm{n}}(0) R_{\mathrm{k}}(0) \iint \lambda\left(x_{\mathrm{n}} y_{\mathrm{n}}\right) d S_{\mathrm{n}} . \\
& \iint \lambda\left(\xi_{\mathrm{k}} \eta_{\mathrm{k}}\right) d S_{\mathrm{k}} M\left(x_{\mathrm{n}} y_{\mathrm{n}} \xi_{\mathrm{k}} \eta_{\mathrm{k}}\right) \\
& =\frac{\int \mathcal{S} \lambda\left(x_{\mathrm{n}} y_{\mathrm{n}}\right) d S_{\mathrm{n}} \int \mathcal{S} \lambda\left(\xi_{\mathrm{k}} \eta_{\mathrm{k}}\right) d S_{\mathrm{k}} M\left(x_{\mathrm{n}} y_{\mathrm{n}} \xi_{\mathrm{k}} \eta_{\mathrm{k}}\right)}{\int \mathcal{S} \lambda\left(x_{\mathrm{n}} y_{\mathrm{n}}\right) d S_{\mathrm{n}}}
\end{aligned}
$$

If $\lambda$ is constant over $S_{\mathrm{n}}$ and $S_{\mathrm{k}}$ then

$$
L_{\mathrm{nk}}=\frac{\int \mathcal{S M}\left(x_{\mathrm{n}} y_{\mathrm{n}} \xi_{\mathrm{n}} \eta_{\mathrm{n}}\right) d x_{\mathrm{n}} d y_{\mathrm{n}} d \xi_{\mathrm{k}} d \eta_{\mathrm{k}}}{S_{\mathrm{n}} S_{\mathrm{k}}}
$$

If, further, there are no ferromagnetic materials in the system $L_{\mathrm{nk}}$ reduces to twice the negative of the geometric mean distance of the sections $S_{\mathrm{n}}$ and $S_{\mathrm{k}}$. The approximations have been carried far enough to show, therefore, that the functions of frequency $R_{\mathrm{nk}}$ and $L_{\mathrm{nk}}$ reduce (for low frequencies) to their ordinary values, which are familiar in the discussion of linear circuits.

\section{THE PROPAGATION CONSTANT $\gamma$. ATTENUATION AND PHASE VELOCITY}

\section{DETERMINANTAL EQUATION FOR $\gamma$}

The attenuation $b$ and phase velocity $V$ are determined by the equation (1) when the complex propagation constant $\gamma$ is known. This must be so chosen as to render compatible the two systems of equations (30) and (67).

$$
\begin{gathered}
\gamma I_{\mathrm{n}}=\sum_{k=1}^{N-1} \alpha_{\mathrm{nk}}\left(c_{\mathrm{k}}-c_{\mathrm{N}}\right) \\
I_{\mathrm{n}}=\gamma \sum_{k=1}^{N-1} \beta_{\mathrm{nk}}\left(c_{\mathrm{k}}-c_{\mathrm{N}}\right)
\end{gathered}
$$

$$
\left\{\begin{array}{c}
\text { for } n=1,2,3 \cdots \cdots \\
\text { where } \alpha_{\mathrm{nk}}=\alpha_{\mathrm{kn}}, \sum_{k=1}^{N} \alpha_{\mathrm{nk}}=0 \\
\beta_{\mathrm{nk}}=\beta_{\mathrm{nk}}, \sum_{k=1}^{N} \beta_{\mathrm{nk}}=0
\end{array}\right.
$$

Eliminating $I_{n}$ gives $N-1$ linear homogeneous relations of the type

$$
\sum_{k=1}^{N-1}\left(\alpha_{\mathrm{nk}}-\gamma^{2} \beta_{\mathrm{nk}}\right)\left(c_{\mathrm{k}}-c_{\mathrm{N}}\right)=0 n=1,2,3 \cdots N-1
$$


They will be compatible if and only if $\gamma^{2}$ is a root of the algebraic equation of degree $N-1$ in $\gamma^{2}$, obtained by placing the determinant of their coefficients equal to zero. That is, if

then $\gamma^{2}$ must be a root of

$$
\delta_{\mathrm{nk}} \equiv \alpha_{\mathrm{nk}}-\gamma^{2} \beta_{\mathrm{nk}}
$$

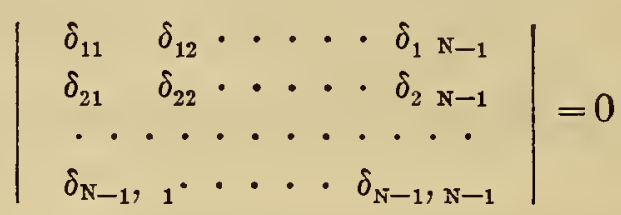

Since $\sum_{k=1}^{N} \delta_{\mathrm{nk}}=0$, one may, by adding all the other rows to the first row, and all the other columns to the first column, show that the equation is the same as if $c_{1}$, or any other $c_{n}$ had been taken as a reference potential instead of $c_{\mathrm{N}}$. Another form of this equation will be obtained if one makes all of the $N$ equations (80), namely,

$$
\gamma c_{\mathrm{B}}-i p A(\infty)=\sum_{k=1}^{N} z_{\mathrm{sk}} \mathrm{I}_{\mathrm{k}} \cdots \cdots s=1,2,3 \cdots N
$$

compatible with all of the $N$ equations (20), Section III.

Since

$$
\gamma^{2} I_{\mathrm{n}}=\sum_{s=1}^{N} \alpha_{\mathrm{ns}} \gamma c_{\mathrm{s}}
$$

$$
\sum_{s=1}^{N} \alpha_{\mathrm{ns}}=0 \text { for } n=1,2,3 \cdots \cdots N
$$

this may be multiplied by $i p A(\infty)$ and subtracted from the above giving

$$
\gamma^{2} I_{\mathrm{n}}=\sum_{s=1}^{N} \alpha_{\mathrm{ns}}\left[\gamma c_{\mathrm{s}}-i p A(\infty)\right] \cdots n=1,2,3 \cdots N
$$

Substituting in these equations the value of $\gamma c_{s}-i p A(\infty)$ from the above gives $N$ linear homogeneous relations that must exist between the $N$ currents, namely,

where

$$
\gamma^{2} I_{\mathrm{n}}=\sum_{k=1}^{N} Z_{\mathrm{nk}} \mathrm{I}_{\mathrm{k}} \cdots \cdots n=1,2,3 \cdots \cdots N
$$

$$
Z_{\mathrm{nk}} \equiv \sum_{s=1}^{N} \alpha_{\mathrm{ns}} z_{s \mathrm{k}} \text { so that } \sum_{n=1}^{N} Z_{\mathrm{nk}}=0 \text { for } k=1,2,3 \cdots N
$$


These equations will be compatible if and only if $\gamma^{2}$ is a root of

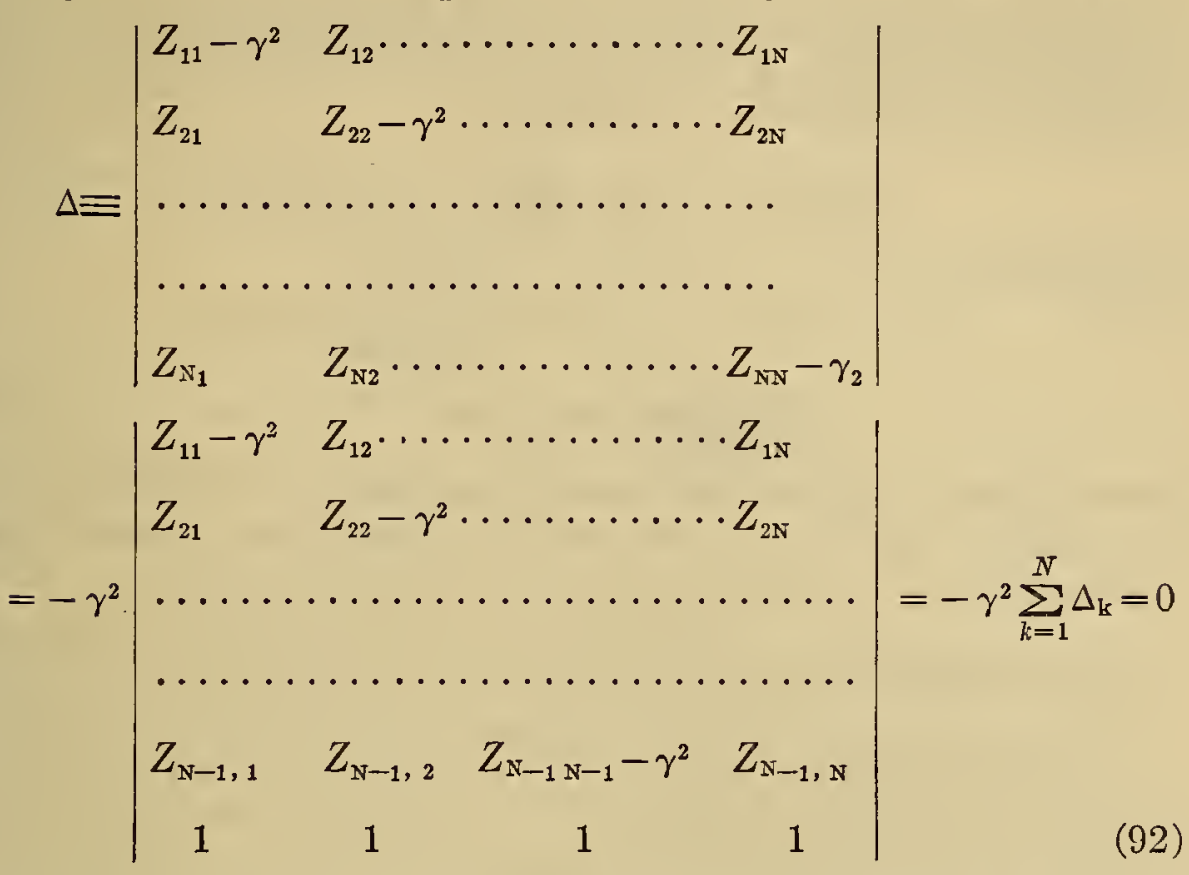

where $\Delta_{\mathrm{k}}$ is the minor formed from $\Delta$ by suppressing the $k^{\text {th }}$ column and last row.

The second form of determinant is obtained by adding all the first $N-1$ rows of $\Delta$ to the lastone and noting that $\sum_{n=1}^{N} Z_{\mathrm{nk}}=0, k=1,2,3 \cdots N$. The row whose elements are unity could thus be placed in any horizontal line of $\Delta$. Thus, $\Delta=0$ is the same equation as that obtained by combining the relation $\sum_{1}^{N} I_{\mathrm{n}}=0$ with any $N-1$ of the relations (4). There will be $N-1$ values of $\gamma^{2}$ in addition to the value $\gamma^{2}=0$, which are roots of this equation. The latter leads to no possible solution; that is, a zero field everywhere.

If $\gamma^{2}$ is a root of $\Delta=0$ and any $N-1$ of the equations be solved for $N-1$ of the currents in terms of one of them, this leads to the relation

or

$$
\frac{I_{1}}{\Delta_{1}}=\frac{I_{2}}{\Delta_{2}}=\cdots \cdots=\frac{I_{\mathbb{N}}}{\Delta_{\mathrm{N}}}=D
$$

where $D$ is an arbitrary constant. The set of equations

$$
\gamma c_{\mathbf{n}}-i p A(\infty)=\sum_{k=1}^{N} z_{\mathrm{nk}} I_{\mathrm{k}}=D \sum_{k=1}^{N} z_{\mathrm{nk}} \Delta_{\mathrm{k}} \cdots n=1,2,3 \cdots N
$$

gives any potential difference

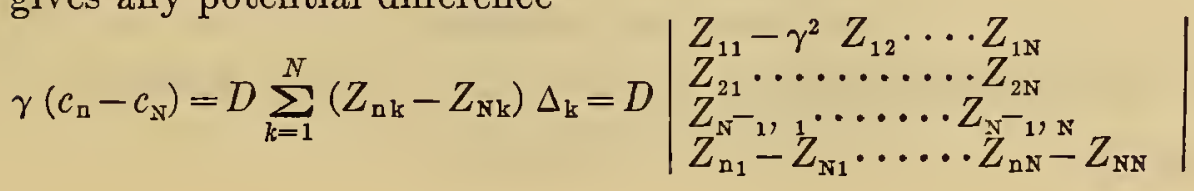


which shows that $\gamma$ and the reactance of the line may be found without computing $A(\infty)$. Its value, however, may be found in terms of $\phi(\infty)$ by multiplying each equation of type (80) by $\phi_{s}(\infty)$ and summing with respect to $s$, noting that

This gives

$$
\sum_{s=1}^{N} \phi_{\mathrm{s}}(\infty)=1 \text { and } \gamma \sum_{s=1}^{N} \phi_{\mathrm{s}}(\infty) c_{\mathrm{s}}=\gamma \phi(\infty)
$$

$$
\begin{aligned}
E(\infty) & =\gamma \phi(\infty)-i p A(\infty)=\sum_{n=1}^{N} \sum_{k=1}^{N} \phi_{\mathrm{n}}(\infty) z_{\mathrm{nk}} I_{\mathrm{k}} \\
& =D \sum_{n=1}^{N} \sum_{k=1}^{N} \phi_{\mathrm{n}}(\infty) z_{\mathrm{nk}} \Delta_{\mathrm{k}}
\end{aligned}
$$

Except for the fact that the terms $Z_{\mathrm{n} k}$ are functions of the frequency, the equation (92) for $\gamma$ is the same as in the case of linear circuits, and it reduces to the same form with vanishing frequency.

\section{DETERMINATION OF CONSTANTS TO FIT TERMINAL CONDITIONS}

For each value of $\gamma^{2}$ which is a root of $\Delta=0$, there are two values $\pm \gamma$ corresponding to a forward and backward wave, each containing an arbitrary constant $D$. In general, there will be $2 N-2$ wave types and this number of constants $D$. It is evident that if the circuit equations involving the currents, potential differences and impedances of the terminal apparatus are written for all the independent modes of connection of the cylinders at the terminal $z=0$, and $z=l$, these with (94) and (95) will afford the necessary and sufficient number of relations to determine the $2 N-2$ constants of type $D$ and hence to completely determine the field. There are $N-1$ independent modes of connecting the terminals of $N$ cylinders at each end.

\section{SPECIAL CASES}

(a) Case of Two Conductors-

$$
\begin{aligned}
& \alpha_{11}=\alpha_{22}=-\alpha_{12}=-\alpha_{21}=G_{12}+i p C_{12} \\
& z_{12}=z_{21}\left\{\begin{array}{l}
R_{12}=R_{21} \\
L_{12}=L_{21}
\end{array}\right. \\
& Z_{11}=-Z_{21}=\alpha_{11} z_{11}+\alpha_{12} z_{21}=\left(G_{12}+i p C_{12}\right)\left(z_{11}-z_{12}\right) \\
& Z_{22}=-Z_{12}=\alpha_{21} z_{12}+\alpha_{22} z_{22}=\left(G_{12}+i p C_{12}\right)\left(z_{22}-z_{12}\right) \\
& \gamma^{2}=Z_{11}-Z_{12}=\left(G_{12}+i p C_{12}\right)\left(z_{11}+z_{22}-2 z_{12}\right) \\
& \gamma c_{1}-i p A(\infty)=z_{11} I_{1}+z_{12} I_{2}=\left(z_{11}-z_{12}\right) I_{1} \\
& \gamma c_{2}-i p A(\infty)=z_{21} I_{1}+z_{22} I_{2}=\left(z_{12}-z_{23}\right) I_{1} \\
& \gamma\left(c_{1}-c_{2}\right)=\left(z_{11}+z_{22}-2 z_{12}\right) I_{1}=(R+i p L) I_{1}
\end{aligned}
$$

where $R$ and $L$ denote resistance and inductance of the line per unit length and are defined by

$$
\begin{gathered}
R \equiv R_{11}+R_{22}-2 R_{12} \\
L \equiv L_{11}+L_{22}-2 L_{12} \\
\gamma^{2}=\left(G_{12}+i p C_{12}\right)(R+i p L)
\end{gathered}
$$

$\frac{1}{G_{12}}$ and $C_{12}$ are the insulation resistance and capacity, respectively, between the two conductors per unit length. 
(b) Uniform Proximity Effect.-If the sections of the conductors are all small compared to their distance apart, the general equation (36), Section IV, breaks up into $N$ independent integral equations of the type.

$$
\begin{gathered}
E\left(x_{\mathrm{n}} y_{\mathrm{n}}\right)+i p \mathcal{S} \mathcal{S} M\left(x_{\mathrm{n}} y_{\mathrm{n}} \xi_{\mathrm{n}} \eta_{\mathrm{n}}\right) \lambda\left(\xi_{\mathrm{n}} \eta_{\mathrm{n}}\right) E\left(\xi_{\mathrm{n}} \eta_{\mathrm{n}}\right) d \xi_{\mathrm{n}} d \eta_{\mathrm{n}} \\
=-i p \Sigma^{\prime} M\left(x_{\mathrm{n}} y_{\mathrm{n}} \xi_{\mathrm{k}} \eta_{\mathrm{k}}\right) \mathcal{S} \mathcal{S} \lambda\left(\xi_{\mathrm{k}} \eta_{\mathrm{k}}\right) E\left(\xi_{\mathrm{k}} \eta_{\mathrm{k}}\right) d \xi_{\mathrm{k}} d \eta_{\mathrm{k}} \\
+\gamma c_{\mathrm{n}}-i p A(\infty)
\end{gathered}
$$

where $\sum_{k}^{\prime}$ denotes that the term corresponding to $k=n$ is omitted from the summation. These terms may be written $i p \sum_{k}^{\prime} L_{\mathrm{nk}} I_{\mathrm{k}}$ where

$$
\begin{aligned}
L_{\mathrm{nk}} & =L_{\mathrm{kn}}=M\left(x_{\mathrm{n}} y_{\mathrm{n}} \xi_{\mathrm{k}} \eta_{\mathrm{k}}\right)=\text { a real constant } \\
& =-2 \log r\left(x_{\mathrm{n}} y_{\mathrm{n}} \xi_{\mathrm{k}} \eta_{\mathrm{k}}\right) \text { if all materials are nonmagnetic }
\end{aligned}
$$

The integral on the left side of (99) is taken over the section of the $n^{\text {th }}$ conductor, the effect of the proximity of other conductors being uniform over this section and represented by the summation on the right side. The entire second member of (99) is constant, say $D_{n}$, over the section $S_{\mathrm{n}}$. This equation may be called the "skin effect equation," since the proximity of other conductors does not affect the relative distribution of current in any one. Its solution will be of the form

$$
E\left(x_{\mathrm{n}} y_{\mathrm{n}}\right)=D_{\mathrm{n}} F_{\mathrm{n}}\left(x_{\mathrm{n}} y_{\mathrm{n}} i p\right)
$$

Multiplying by $\gamma_{\mathrm{n}} d x_{\mathrm{n}} d y_{\mathrm{n}}$ and integrating over the section $S_{\mathrm{n}}$ gives

$$
I_{\mathrm{n}}=D_{\mathrm{n}} \int \mathcal{S} \gamma\left(x_{\mathrm{n}} y_{\mathrm{n}}\right) F_{\mathrm{n}}\left(x_{\mathrm{n}} y_{\mathrm{n}} i p\right) d x_{\mathrm{n}} d y_{\mathrm{n}}
$$

Since this integral can never vanish (equation 58), the real functions of frequency $R_{\mathrm{nn}}$ and $L_{\mathrm{nn}}$ may be defined by

$$
R_{\mathrm{nn}}+i p L_{\mathrm{nn}}=\frac{1}{\iint \lambda_{\mathrm{n}} F_{\mathrm{n}}\left(x_{\mathrm{n}} y_{\mathrm{n}} i p\right) d S_{\mathrm{n}}}
$$

If we also define $R_{\mathrm{nk}}$ to be zero if $n \neq k$ and replace $D_{\mathrm{n}}$ in (101) by its value

$$
\gamma c_{\mathrm{n}}-i p A(\infty)-i p \sum_{k}^{\prime} L_{\mathrm{nk}} I_{\mathrm{k}}
$$

it reduces to the general form (80) Section IV

$$
\gamma c_{2}-i p A(\infty)=\sum_{k=1}^{N}\left(R_{\mathrm{nk}}+i p L_{\mathrm{nk}}\right) I_{\mathrm{k}}=\sum_{k=1}^{N} z_{\mathrm{nk}} I_{\mathrm{k}}
$$

The completion of the problem is the same as that outlined in general in this section; the coefficients of leakage and capacity must be found in order to write out the determinant for $\gamma^{2}$. 


\section{ENERGY RELATIONS AND MEAN VALUES}

Suppose $\phi, A$, and $E$ have been found in the form $\phi^{\prime}+i \phi^{\prime \prime}$, $A^{\prime}+i A^{\prime \prime}, E^{\prime}+i E^{\prime \prime}$ where $\phi^{\prime} \phi^{\prime \prime}$, etc., are real functions of $x y$ and $p$. The instantaneous value of any of the above quantities is found by supplying the exponential factor $e^{\mathrm{ipt}-\gamma \mathrm{z}}=e^{-\mathrm{bz}} \cdot e^{\mathrm{ip}\left(\mathrm{t}-\frac{\mathrm{z}}{\mathrm{v}}\right)}$ and then taking the real part.

If $P=P^{\prime}+i P^{\prime \prime}$ and $Q=Q^{\prime}+i Q^{\prime \prime}$ are any two typical quantities of this kind, the time average of the product of the instantaneous (real) values of $P$ and $Q$ is found to be

$$
e^{-2 \mathrm{bz}}\left(\frac{P^{\prime} Q^{\prime}+P^{\prime \prime} Q^{\prime \prime}}{2}\right)
$$

The time averages which will be dealt with in this section presuppose a single type of wave corresponding to one ralue of $\gamma$ only, and obviously do not apply when more than one type of waves coexist since it is the field components and not the energies that are additive.

Consider the medium between two planes perpendicular to the $z$ axis at $z$ and $z+d z$. Denote the time average of the rate of flow of energy through the plane $z=$ constant, in the positive $z$ direction by $e^{-2 \mathrm{kz}} \bar{F}$. There is no flow of energy in this dircction in conductors, it is all in the dielectric and flows laterally into the conductors. Denote the mean rate of heating in all the conductors between these two planes by $e^{-2 \mathrm{bz}} d z \overline{W_{\mathrm{c}}}$, that in the dielectric by $e^{-2 \mathrm{bz}} d z \overline{W_{\mathrm{d}}}$. Also let $e^{-2} \mathrm{bz} d z \bar{U}$ and $e^{-2 \mathrm{bz}} d z \bar{T}$ denote, respectively, the time average of the electrical energy in the dielectric, and of the electrokinetic energy between these two planes. The former is localized in the dielectric only.

The definitions of these quantities lead to the forms

$$
\begin{aligned}
& \bar{F}=\frac{1}{8 \pi} \iint\left[\frac{\partial \phi^{\prime}}{\partial x} \cdot \frac{\partial A^{\prime}}{\partial x}+\frac{\partial \phi^{\prime}}{\partial y} \cdot \frac{\partial A^{\prime}}{\partial y}+\frac{\partial \phi^{\prime \prime}}{\partial x} \cdot \frac{\partial A^{\prime \prime}}{\partial x}+\frac{\partial \phi^{\prime \prime}}{\partial y} \cdot \frac{\partial A^{\prime \prime}}{\partial y}\right] d S \\
& \bar{W}_{\mathrm{d}}=\iint \lambda\left[\frac{\left.\left(\frac{\partial \phi^{\prime}}{\partial x}\right)^{2}+\left(\frac{\partial \phi^{\prime}}{\partial y}\right)^{2}+\left(\frac{\partial \phi^{\prime \prime}}{\partial x}\right)^{2}+\frac{\partial \phi^{\prime \prime}}{\partial y}\right)^{2}}{2}\right] d S \\
& \bar{U}=\frac{1}{3 \pi} \iint \frac{\mathrm{k}}{c^{2}}\left[\frac{\left(\frac{\partial \phi^{\prime}}{\partial x}\right)^{2}+\left(\frac{\partial \phi^{\prime}}{\partial y}\right)^{2}+\left(\frac{\partial \phi^{\prime \prime}}{\partial x}\right)^{2}+\left(\frac{\partial \phi^{\prime \prime}}{\partial y}\right)^{2}}{2}\right] d S
\end{aligned}
$$

where the integration is over all dielectric sections in the plane.

$$
\bar{W}_{\mathrm{c}}=\int \mathcal{S} \lambda\left(\frac{\mathrm{E}^{\prime 2}+\mathrm{E}^{\prime \prime 2}}{2}\right) d S
$$

over all conducting sections. 


$$
T=\frac{1}{8 \pi} \iint \frac{1}{\mu}\left[\frac{\left(\frac{\partial A^{\prime}}{\partial x}\right)^{2}+\left(\frac{\partial A^{\prime}}{\partial y}\right)^{2}+\left(\frac{\partial A^{\prime \prime}}{\partial x}\right)^{2}+\left(\frac{\partial A^{\prime \prime}}{\partial y}\right)^{2}}{2}\right] d S
$$

over the entire plane

$$
=\frac{1}{2} \iint \lambda\left(\frac{E^{\prime} A^{\prime}+E^{\prime \prime} A^{\prime \prime}}{2}\right) d S
$$

over all conducting sections.

The justification of these expressions of $\bar{W}_{\mathrm{d}}$ and $\bar{U}$ lies in the fact that $\left(\lambda+\frac{i p k}{4 \pi c^{2}}\right) E^{2}$ is infinitesimal compared to

$$
\left(\lambda+\frac{i p k}{4 \pi c^{2}}\right)\left[\left(\frac{\partial \phi}{\partial x}\right)^{2}+\left(\frac{\partial \phi}{\partial y}\right)^{2}\right]
$$

The formula is correct to first approximations even in those cases where the constant $E(\infty)$ is not zero, for a second approximation would make $E$ vanish at infinity without affecting the first approximation to current distribution in conductors, or to $x y$ components of leakage and capacity current in dielectrics.

The differential equations and boundary conditions which $\phi$ and $A$ satisfy lead, by simple transformations of the above integrals, to the following values, (if $\gamma \equiv b+\frac{i p}{V}$ )

$$
\begin{aligned}
& \bar{F}=\frac{1}{2} \sum_{k=1}^{N}\left(c^{\prime}{ }_{\mathrm{k}} I^{\prime}{ }_{\mathrm{k}}+c^{\prime \prime}{ }_{\mathrm{k}} I^{\prime \prime}{ }_{\mathrm{k}}\right) \\
& \bar{W}_{\mathrm{d}}=\frac{1}{2} \sum_{k=1}^{N}\left[c^{\prime}{ }_{\mathrm{k}}\left(G^{\prime}{ }_{\mathrm{k}}-p Q^{\prime \prime}{ }_{\mathrm{k}}\right)+c^{\prime \prime}{ }_{\mathrm{k}}\left(G^{\prime \prime}{ }_{\mathrm{k}}+p Q^{\prime}{ }_{\mathrm{k}}\right)\right] \\
& \bar{U}=\frac{1}{4 p} \sum_{k=1}^{N}\left[c^{\prime}{ }_{\mathrm{k}}\left(G^{\prime \prime}{ }_{\mathrm{k}}+p Q^{\prime}{ }_{\mathbf{k}}\right)-c^{\prime \prime}{ }_{\mathrm{k}}\left(G_{\mathrm{T}_{\mathrm{k}}}-p Q^{\prime \prime}{ }_{\mathrm{k}}\right)\right] \\
& \bar{W}_{\mathrm{c}}=\frac{1}{2} \sum_{k=1}^{N}\left[b\left(c^{\prime}{ }_{\mathrm{k}} I^{\prime}{ }_{\mathrm{k}}+c^{\prime \prime}{ }_{\mathrm{k}} I^{\prime \prime}{ }_{\mathrm{k}}\right)+\frac{p^{-}}{V}\left(c^{\prime}{ }_{\mathrm{k}} I^{\prime \prime}{ }_{\mathrm{k}}-c^{\prime \prime}{ }_{\mathrm{k}} I^{\prime}{ }_{\mathrm{k}}\right)\right] \\
& \bar{T}=\frac{1}{4 p} \sum_{k=1}^{N} \frac{p}{V}\left[\left(c^{\prime}{ }_{\mathrm{k}} I^{\prime}{ }_{\mathrm{k}}+c^{\prime \prime}{ }_{\mathrm{k}} I^{\prime \prime}{ }_{\mathrm{k}}\right)-b\left(c^{\prime}{ }_{\mathrm{k}} I^{\prime \prime}{ }_{\mathrm{k}}-c^{\prime \prime}{ }_{\mathrm{k}} I^{\prime}{ }_{\mathrm{k}}\right)\right]
\end{aligned}
$$

Since

$$
\gamma I_{\mathbf{k}}=g_{\mathrm{k}}+i p q_{\mathrm{k}}\left\{\begin{array}{l}
b I^{\prime}{ }_{\mathbf{k}}-\frac{p}{V} I^{\prime \prime}{ }_{\mathbf{k}}=G^{\prime}{ }_{\mathbf{k}}-p Q^{\prime \prime}{ }_{\mathbf{k}} \\
\frac{p}{V} I^{\prime}{ }_{\mathbf{k}}+b I^{\prime \prime}{ }_{\mathrm{k}}=G^{\prime \prime}{ }_{\mathrm{k}}+p Q^{\prime}{ }_{\mathbf{k}}
\end{array}\right.
$$

so that the above expressions for $\bar{W}_{d}$ and $\bar{U}$ may be put in the form

$$
\begin{aligned}
& \bar{W}_{d}=\frac{1}{2} \sum_{k=1}^{N}\left[b\left(c^{\prime}{ }_{\mathrm{k}} I^{\prime}{ }_{\mathrm{k}}+c^{\prime \prime}{ }_{\mathrm{k}} I^{\prime \prime}{ }_{\mathrm{k}}\right)-\frac{p}{V}\left(c^{\prime}{ }_{\mathrm{k}} I^{\prime \prime}{ }_{\mathrm{k}}-c^{\prime \prime}{ }_{\mathrm{k}} I^{\prime}{ }_{\mathrm{k}}\right)\right] \\
& \bar{U}_{d}=\frac{1}{4 p} \sum_{k=1}^{N}\left[\frac{p}{V}\left(c^{\prime}{ }_{\mathrm{k}} I_{\mathrm{k}}+c^{\prime \prime}{ }_{\mathrm{k}} I^{\prime \prime}{ }_{\mathrm{k}}\right)+b\left(c^{\prime}{ }_{\mathrm{k}} I^{\prime \prime}{ }_{\mathrm{k}}-c^{\prime \prime}{ }_{\mathrm{k}} I^{\prime}{ }_{\mathrm{k}}\right)\right]
\end{aligned}
$$


From these expressions it is evident that

and

$$
W_{\mathrm{c}}-W_{d}=\frac{2 p^{2}}{b V}(\bar{U}-\bar{T})
$$

$$
\bar{F}=\frac{\bar{W}_{\mathrm{c}}+\bar{W}_{\mathrm{d}}}{2 b}=V(\bar{U}+\bar{T})
$$

That is, the mean flow of energy $e^{-2 \mathrm{bz}} \bar{F}$ through the plane $z=$ constant is equal to the phase velocity $V$, multiplied by the density per unit length along $z$ of the total electric and magnetic energy $e^{-2 b z}(\widetilde{U+T} T)$. The last equation may also be put in the form

$$
-\frac{d}{d z}\left(e^{-2 \mathrm{bz}} \bar{F}\right) d z=e^{-2 \mathrm{bz}}\left(\bar{W}_{\mathrm{c}}+\bar{W}_{\mathrm{d}}\right) d z
$$

which states that the excess of mean energy flow into the medium between the planes $z$ and $z+d z$ is equal to the mean rate of dissipation of energy between these planes.

It may be noted that the dissipation of energy $\bar{W}_{d}$ in the dielectric may be of the same order of magnitude as that in conductors $W_{\mathrm{d}}$. If these two should be equal, then the mean electrical and magnetic energies would be equal as in the case of homogeneous waves.

The relation

enables us to write

$$
\gamma c_{\mathbf{k}}-i p A(\infty)=\sum_{n=1}^{N}\left(R_{\mathrm{nk}}+i p L_{\mathrm{nk}}\right) I_{\mathbf{n}}
$$

The relation

$$
\begin{aligned}
\bar{W}_{\mathrm{c}} & =\sum_{n=1}^{N} \sum_{k=1}^{N} R_{\mathrm{nk}}\left(\frac{I^{\prime}{ }_{\mathrm{n}} I_{\mathrm{k}}+I^{\prime \prime}{ }_{\mathrm{n}} I^{\prime \prime}{ }_{\mathrm{k}}}{2}\right) \\
\bar{T} & =\frac{1}{2} \sum_{n=1}^{N} \sum_{k=1}^{N} L_{\mathrm{nk}}\left(\frac{I^{\prime}{ }_{\mathrm{n}}{ }^{\prime}{ }_{\mathrm{k}}+I^{\prime \prime}{ }_{\mathrm{n}} I^{\prime \prime}{ }_{\mathrm{k}}}{2}\right)
\end{aligned}
$$

leads to

$$
\gamma I_{\mathrm{k}}=g_{\mathrm{k}}+i p q_{\mathrm{k}}=\sum_{n=1}^{N=1}\left(G_{\mathrm{nk}}+i p C_{\mathrm{nk}}\right)\left(c_{\mathrm{N}}-c_{\mathrm{n}}\right)
$$

$$
\begin{aligned}
& \bar{W}_{d}=\sum_{n=1}^{N-1} \sum_{k=1}^{N-1} G_{\mathrm{nk}}\left[\frac{\left(c^{\prime}{ }_{\mathrm{n}}-c^{\prime}{ }_{\mathrm{X}}\right)\left(c^{\prime}{ }_{\mathrm{N}}-c^{\prime}{ }_{\mathrm{k}}\right)+\left(c^{\prime \prime}{ }_{\mathrm{n}}-c^{\prime \prime}{ }_{\mathrm{N}}\right)\left(c^{\prime \prime}{ }_{\mathrm{N}}-c^{\prime \prime}{ }_{\mathrm{k}}\right)}{2}\right] \\
& \bar{O}=\frac{1}{2} \sum_{n=1}^{N-1} \sum_{k=1}^{N-1} C_{\mathrm{nk}}\left[\frac{\left(c^{\prime}{ }_{\mathrm{n}}-c^{\prime}{ }_{\mathrm{N}}\right)\left(c^{\prime}{ }_{\mathrm{N}}-c^{\prime}{ }_{\mathrm{k}}\right)+\left(c^{\prime \prime}{ }_{\mathrm{n}}-c^{\prime \prime}{ }_{\mathrm{N}}\right)\left(c^{\prime \prime}{ }_{\mathrm{N}}-c^{\prime \prime}{ }_{\mathrm{k}}\right)}{2}\right]
\end{aligned}
$$

If the new real quantities $R_{\mathrm{n}}$ and $L_{\mathrm{n}}$ be defined by the equation

$$
R_{\mathrm{n}}+i p L_{\mathrm{n}} \equiv \frac{\sum_{k=1}^{N} z_{\mathrm{nk}} \triangle_{\mathrm{k}}}{\triangle_{\mathrm{n}}}=\frac{\sum_{k=1}^{N}\left(R_{\mathrm{nk}}+i p L_{\mathrm{nk}}\right) \triangle_{\mathrm{k}}}{\triangle_{\mathrm{n}}}
$$


Then

$$
\gamma \epsilon_{\mathrm{n}}-i p A(\infty)=\left(R_{\mathrm{n}}+i p L_{\mathrm{n}}\right) I_{\mathrm{n}}=\boldsymbol{\Sigma}\left(R_{\mathrm{nk}}+i p L_{\mathrm{nk}}\right) I_{\mathrm{k}}
$$

and this gives the simpler forms

$$
\begin{aligned}
& \bar{W}_{\mathrm{c}}=\sum_{n=1}^{N} R_{\mathrm{n}}\left[\frac{I_{\mathrm{n}}{ }^{2}+I^{\prime \prime}{ }_{\mathrm{n}}{ }^{2}}{2}\right] \\
& \bar{T}=\frac{1}{2} \sum_{n=1}^{N} L_{\mathrm{n}}\left[\frac{I_{\mathrm{n}}{ }^{2}+I^{\prime \prime}{ }_{\mathrm{n}}{ }^{2}}{2}\right]
\end{aligned}
$$

Such expressions may, however, be misleading on account of their simplicity. For example, the heating in the $n^{\text {th }}$ conductor is not

in general, but is

$$
R_{\mathrm{n}}\left[\frac{I^{\prime}{ }^{2}+I^{\prime \prime}{ }^{2}}{2}\right]
$$

$$
\iint \lambda\left(E^{\prime 2}+E^{\prime \prime 2}\right) d S_{\mathrm{n}}
$$

\section{EXAMPLES AND APPLICATIONS}

\section{CASE OF TWO CONDUCTORS WITH CIRCULAR SYMMETRY}

The only interest that can be attached here to such a well-known problem is to show that the integral equation for say $\omega_{1}$ does uniquely determine $\omega_{1}$ and hence $\psi_{1}=\phi_{1}-\omega_{1}$ without reference to any boundary conditions. Hence, the simplest case is taken of a circular cylinder of radius $r_{1}$ with a return conductor in the shape of a concentric shell of inner and outer radii $r_{2}$ and $r_{3}$, respectively, both conductors being nonmagnetic and having the same conductivity, and the dielectric being air.

In this case if $h^{2}=-4 \pi i p \lambda$

\begin{tabular}{|l|c|c|c|}
\hline$r_{1}>r \geq 0$ & $r_{2}>r>r_{1}$ & $r_{3}>r>r_{3}$ & $r>r_{3}$ \\
\hline$\phi_{1}=1$ & $\frac{\log \frac{r}{r_{3}}}{\log \frac{r_{1}}{r_{2}}}$ & 0 & 0 \\
\hline$\psi_{1}=1+C_{1} J_{0}(h r)$ & $C_{3}+C_{3} \log r$ & $C_{4} J_{0}(h r)+C_{5} \mathrm{~K}_{0}(h r)$ & $C_{6} \log r$ \\
\hline
\end{tabular}

These forms are required by the differential equation for $\psi_{1}(76 a)$. Writing out the equations, the boundary conditions for $\psi(76 \mathrm{~b})$ and (76c), and eliminating the constants $C_{2}, C_{3}$, and $\mathrm{C}_{6}$, which correspond to the dielectric regions, letting

$$
\begin{aligned}
& P(x) \equiv J_{\circ}(x)-\left(\log \frac{x}{h}\right) x J^{\prime}{ }_{\circ}(x) \\
& Q(x) \equiv K_{\circ}(x)-\left(\log \frac{x}{h}\right) x K^{\prime}(x)
\end{aligned}
$$

gives the three equations

$$
\begin{aligned}
& -P\left(x_{1}\right) C_{1}+P\left(x_{2}\right) C_{4}+Q\left(x_{2}\right) C_{5}=1 \\
& -x_{1} J^{\prime}{ }_{o}\left(x_{1}\right) C_{1}+x_{2} J_{0}^{\prime}\left(x_{2}\right) C_{4}+x_{2} K^{\prime}{ }_{0}\left(x_{2}\right) C_{3}=0 \\
& \quad O C_{1}+P\left(x_{3}\right) C_{4}+Q\left(x_{3}\right) C_{3}=0
\end{aligned}
$$


The determinant of these three equations can not vanish for any real value of the frequency, hence they determine $C_{1}, C_{4}$, and $C_{5}$.

To show that the integral equation for $\omega_{1}$ leads to the same values of these three constants directly, express $M\left(r r^{\prime}\right)$ in the form

$$
M\left(r r^{\prime}\right)=-2 \log R\left(r r^{\prime}\right)=-2\left[a_{\circ}\left(r r^{\prime}\right)-\sum_{n=1}^{\infty} a_{\mathrm{n}}\left(r r^{\prime}\right) \frac{\cos n\left(\theta-\theta^{\prime}\right)}{n}\right]
$$

Where

$$
\begin{aligned}
a_{\mathrm{o}}\left(r r^{\prime}\right) & =\log r \text { if } r^{\prime}<r \\
& =\log r^{\prime} \text { if } r^{\prime}>r \\
a_{\mathrm{n}}\left(r r^{\prime}\right) & =\left(\frac{r^{\prime}}{r}\right)^{\mathrm{n}} \text { if } r^{\prime}<r \\
& =\left(\frac{r}{r^{\prime}}\right)^{\mathrm{n}} \text { if } r^{\prime}>r
\end{aligned}
$$

Since $\omega_{1}$ is a function of $r$ only and not of $\theta$ in both conductors, the integral equation for $\omega_{1}$ reduces to

$$
\omega_{1}(r)+h^{2} \int a_{\mathrm{o}}\left(r r^{\prime}\right) \cdot r^{\prime} \omega_{1}\left(r^{\prime}\right) d r^{\prime}=\phi_{1}(r)=\left\{\begin{array}{l}
1 \text { if } r<r_{1} \\
0 \text { if } r>r_{2}
\end{array}\right.
$$

where the integral is taken over both conductors. This equation has one solution and one only, and if we assume the form

$$
\begin{array}{rr}
\omega_{1}(r)=-C_{1} J_{\circ}(h r) & r<r_{1} \\
=-C_{4} J_{0}(h r)-C_{5} K_{0}(h r) \text { when } r_{2}<r<r_{3}
\end{array}
$$

and substitute this form in the above equation, it requires that

$$
\begin{aligned}
& -P\left(x_{1}\right) C_{1}+\left[P\left(x_{2}\right)-P\left(x_{3}\right)\right] C_{4}+\left[Q\left(x_{2}\right)-Q\left(x_{3}\right)\right] C_{3}=1 \\
& -x_{1} J_{0}\left(x_{1}\right) C_{1}+x_{2} J^{\prime}\left(x_{2}\right) C_{4}+x_{2} K^{\prime}{ }_{0}\left(x_{2}\right) C_{5}=0 \\
& \quad P\left(x_{3}\right) C_{4}+Q\left(x_{3}\right) C_{5}=0
\end{aligned}
$$

By adding the third of these to the first the same set of equations for $C_{1}, C_{4}$, and $C_{5}$ is obtained as was found by satisfying the differential equations and boundary conditions. This shows that the integral equation will uniquely determine the field without any reference to boundary conditions.

It may be noted that $E(\infty)=\gamma \phi(\infty)-i p A(\infty)$ can not vanish in this problem.

In evaluating the integrals to obtain these equations, use may be made of the fact that

$$
\frac{1}{x} \frac{d}{d x}\left[x J_{0}(x)\right]+J_{0}(x)=0 \quad x J_{0}(x)=-\frac{d}{d x}\left[x J^{\prime}{ }_{0}(x)\right]
$$

and

$$
x \log x J_{\mathrm{o}}(x)=-\frac{d}{d x}\left[x \log x{J^{\prime}}_{\mathrm{o}}(x)\right]+\frac{d J_{\mathrm{o}}(x)}{d x}
$$

with similar formulas for $\Pi_{0}(x)$. 
2. CASE OF TWO CIRCULAR, CYLINDRICAL CONDUCTORS OF DIFFERENT CONDUCTIVITY, PERMEABILITY, AND RADIUS, SURROUNDED BY A HOMOGENEOUS, SLIGHTLY CONDUCTING DIELECTRIC. MIXED METHOD

In this example the mixed method of Section IV will be employed.

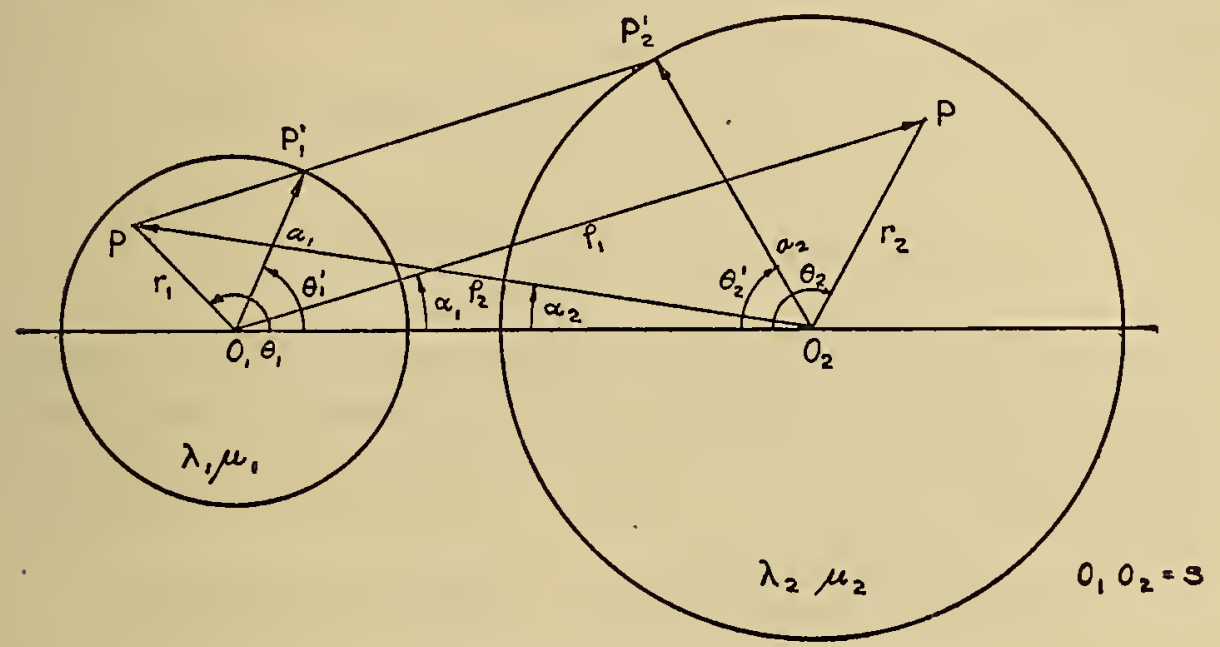

Fig. 2.-Sections of two unequal cylinders

$$
\left\{\begin{array}{l}
h_{1} \equiv \sqrt{2 \pi p \mu_{1} \lambda_{1}}(1-\mathrm{i}) \text { and } x_{1}=h_{1} a_{1}=\sqrt{\frac{2 p \mu_{1}}{R_{1}(0)}}(1-\mathrm{i}) \\
h_{2} \equiv \sqrt{2 \pi p \mu_{2} \lambda_{2}}(1-\mathrm{i}) \text { and } x_{2}=h_{2} a_{2}=\sqrt{\frac{2 p \mu_{2}}{R_{2}(0)}}(1-\mathrm{i})
\end{array}\right.
$$

Assume the form

$$
\left\{\begin{array}{l}
A=\frac{\gamma c_{1}}{i p}-I_{1}\left[C_{\mathrm{o}} J_{\mathrm{o}}\left(h_{1} r_{1}\right)+\sum_{n=1}^{\infty} C_{\mathrm{n}} J_{\mathrm{n}}\left(h_{1} r_{1}\right) \cos n \theta_{1}\right] \text { if } P\left(r_{1} \theta_{1}\right) \text { is in No. } 1 \\
A=\frac{\gamma c_{2}}{i p}-I_{2}\left[D_{0} J_{0}\left(h_{2} r_{2}\right)+\sum_{n=1}^{\infty} D_{\mathrm{n}} J_{\mathrm{n}}\left(h_{2} r_{2}\right) \cos n \theta_{2}\right] \text { if } P\left(r_{2} \theta_{2}\right) \text { is in No. } 2
\end{array}\right.
$$

The equation which must be identically true when the point $P$ is within either circle is (51).

$$
\begin{aligned}
A(\infty) & =\frac{a_{1}}{2 \pi} \int_{0}^{2 \pi} d \theta^{\prime}{ }_{1}\left[A\left(a_{1} \theta^{\prime}{ }_{1}\right) \frac{\partial}{\partial a_{1}} \log R\left(P P^{\prime}{ }_{1}\right)-\frac{\log R\left(P P^{\prime}{ }_{1}\right)}{\mu_{1}}\left(\frac{\partial A}{\partial a_{1}}\right)\left(a_{1} \theta^{\prime}{ }_{1}\right)\right] \\
& +\frac{a_{2}}{2 \pi} \int_{0}^{2 \pi} d \theta^{\prime}{ }_{2}\left[A\left(a_{2} \theta^{\prime}{ }_{2}\right) \frac{\partial}{\partial a_{2}} \log R\left(P P^{\prime}{ }_{2}\right)-\frac{\log R\left(P P^{\prime}{ }_{2}\right)}{\mu_{2}}\left(\frac{\partial A}{\partial a_{2}}\right)\left(a_{2} \theta^{\prime}{ }_{2}\right)\right]
\end{aligned}
$$


In case $1, P$ is inside circle No. 1

$$
\begin{aligned}
& \log R\left(P P^{\prime}{ }_{1}\right)=\log a_{1}-\sum_{k=1}^{\infty}\left(\frac{r_{1}}{a_{1}}\right)^{\mathrm{k}} \frac{\cos k\left(\theta_{1}-\theta_{1}^{\prime}\right)}{k} \\
& \log R\left(P P^{\prime}{ }_{2}\right)=\log \rho_{2}-\sum_{k=1}^{\infty}\left(\frac{a_{2}}{\rho_{2}}\right)^{\mathrm{k}} \frac{\cos k\left(\theta^{\prime}{ }_{2}-\alpha_{2}\right)}{k}
\end{aligned}
$$

In case $2, P$ is within circle No. 2 .

$$
\begin{aligned}
& \log R\left(P P^{\prime}{ }_{1}\right)=\log \rho_{1}-\sum_{k=1}^{\infty}\left(\frac{a_{1}}{\rho_{1}}\right)^{\mathrm{k}} \frac{\cos k\left(\theta_{1}^{\prime}-\alpha_{1}\right)}{k} \\
& \log R\left(P P_{2}^{\prime}\right)=\log a_{2}-\sum_{k=1}^{\infty}\left(\frac{r_{2}}{a_{2}}\right)^{\mathrm{k}} \frac{\cos k\left(\theta_{2}-\theta_{2}^{\prime}\right)}{k}
\end{aligned}
$$

If the expressions (107) for $A$ be used in (108) and the terms be written out for case 1 , the corresponding equation for case 2 may be formed from this by a permutation of the subscripts 1 and 2 and of the constants $C_{n}$ and $D_{n}$. In case 1, the equation (108) becomes

$$
\begin{aligned}
& A(\infty)=\frac{I_{1}}{2 \pi} \int_{0}^{2 \pi} d \theta^{\prime}{ }_{1}\left\{\left[\frac{\gamma c_{1}}{i p I_{1}} C_{\mathrm{o}} J_{\mathrm{o}}\left(x_{1}\right)-\sum_{n=1}^{\infty} C_{\mathrm{n}} J_{\mathrm{n}}\left(x_{1}\right) \cos n \theta^{\prime}{ }_{1}\right] .\right. \\
& \cdot\left[1+\sum_{k=1}^{\infty}\left(\frac{r_{1}}{a_{1}}\right)^{\mathbf{k}} \cos k\left(\theta_{1}-\theta^{\prime}{ }_{1}\right)\right] \\
& +\frac{x_{1}}{\mu_{1}}\left[C_{0} J^{\prime}{ }_{0}\left(x_{1}\right)+\sum_{n=1}^{\infty} C_{n} J^{\prime}{ }_{n}\left(x_{1}\right) \cos n \theta^{\prime}{ }_{1}\right] \text {. }
\end{aligned}
$$

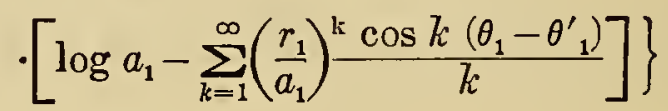

$$
\begin{aligned}
& +\frac{l_{2}}{2 \pi} \int_{0}^{2 \pi} \mathrm{d} \theta^{\prime}{ }_{2}\left\{\left[\frac{\gamma c_{2}}{I_{2}}-D_{\mathrm{o}} J_{\mathrm{o}}\left(x_{2}\right)-\sum_{n=1}^{\infty} D_{\mathrm{n}} J_{\mathrm{n}}\left(x_{2}\right) \cos n \theta_{2}^{\prime}\right]\right. \text {. } \\
& \cdot\left[-\sum_{k=1}^{\infty}\left(\frac{a_{2}}{\rho_{2}}\right)^{k} \cos k\left(\theta_{2}^{\prime}-\alpha_{2}\right)\right] \\
& +\frac{x_{2}}{\mu_{2}}\left[D_{\mathrm{o}} J^{\prime}{ }_{\mathrm{o}}\left(x_{2}\right)+\sum_{n=1}^{\infty} D_{\mathrm{n}} J^{\prime}{ }_{\mathrm{n}}\left(x_{2}\right) \cos n{\theta^{\prime}}_{2}\right] \text {. }
\end{aligned}
$$

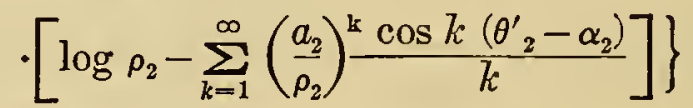

Or, after integrating with respect to $\theta^{\prime}{ }_{1}$ and $\theta^{\prime}{ }_{2}$

$$
\begin{gathered}
A(\infty)=\frac{\gamma c_{1}}{i p}-I_{1} C_{\mathrm{o}}\left[J_{\mathrm{o}}\left(x_{1}\right)-\frac{x_{1} J^{\prime}{ }_{\mathrm{o}}\left(x_{1}\right) \log a_{1}}{\mu_{1}}\right] \\
-\frac{I_{1}}{2} \sum_{n=1}^{\infty} C_{\mathrm{n}}\left[J_{\mathrm{n}}\left(x_{1}\right)+\frac{x_{1}}{\mu_{1}} \frac{J^{\prime}{ }_{\mathrm{n}}\left(x_{1}\right)}{n}\right]\left(\frac{r_{1}}{a_{1}}\right)^{\mathrm{n}} \cos n \theta_{1} \\
+I_{2} D_{\mathrm{o}} \frac{x_{2} J^{\prime}{ }_{0}\left(x_{2}\right) \log \rho_{2}}{\mu_{2}}+\frac{I_{2}}{2} \sum_{k=1}^{\infty} D_{\mathrm{k}}\left[J_{\mathrm{k}}\left(x_{2}\right)-\frac{x_{2} J^{\prime}{ }_{\mathrm{k}}\left(x_{2}\right)}{\mu_{2} k}\right]\left(\frac{a_{2}}{\rho_{2}}\right)^{\mathrm{k}} \cos k \alpha_{2}
\end{gathered}
$$


Introducing into (109) the expansions of the triangle

$$
\begin{gathered}
\log \rho_{2}=\log s-\sum_{n=1}^{\infty}\left(\frac{a_{1}}{s}\right)^{\mathrm{n}}\left(\frac{r_{1}}{a_{1}}\right)^{\mathrm{n}} \frac{\cos n \theta_{1}}{n} \\
\left(\frac{a_{2}}{\rho_{2}}\right)^{\mathrm{k}} \cos k \alpha_{2}=\left(\frac{a_{2}}{s}\right)^{\mathrm{k}}\left\{1+\sum_{n=1}^{\infty} \frac{\lfloor+k-1}{|n| k-1}\left(\frac{a_{1}}{s}\right)^{\mathrm{n}}\left(\frac{r_{1}}{a_{1}}\right)^{\mathrm{n}} \cos n \theta_{1}\right\}
\end{gathered}
$$

gives, after inverting the order of summation of the double sum

$$
\begin{gathered}
O=\frac{\gamma c_{1}-i p A(\infty)}{i p}-I_{1} C_{0}\left[J_{0}\left(x_{1}\right)-\frac{x_{1} J^{\prime}{ }_{0}\left(x_{1}\right) \log a_{1}}{\mu_{1}}\right]+I_{2} D_{0} \frac{x_{2} J^{\prime}{ }_{0}\left(x_{2}\right)}{\mu_{3}} \log s \\
+\frac{I_{2}}{2} \sum_{k=1}^{\infty} D_{\mathrm{k}}\left[J_{\mathbf{k}}\left(x_{2}\right)-\frac{x_{2} J^{\prime}{ }_{\mathbf{k}}\left(x_{2}\right)}{\mu_{2} k}\right]\left(\frac{a_{2}}{s}\right)^{\mathbf{k}} \\
-\sum_{n=1}^{\infty}\left(\frac{r_{1}}{a}\right)^{\mathrm{n}} \cos n \theta_{1}\left\{\frac{I_{1} C_{\mathrm{n}}}{2}\left[J_{\mathrm{n}}\left(x_{1}\right)+\frac{x_{1} J^{\prime}{ }_{\mathrm{n}}\left(x_{1}\right)}{\mu_{1} n}\right]+\frac{I_{2} D_{0} x_{2} J^{\prime}{ }_{0}\left(x_{2}\right)}{\mu_{2} n}\left(\frac{a_{1}}{s}\right)^{\mathrm{n}}\right. \\
\left.\quad-I_{2} \sum_{k=1}^{\infty} \frac{\mid n+k-1}{\underline{n} \mid k-1}\left(\frac{a_{1}}{s}\right)^{\mathrm{n}}\left(\frac{a_{2}}{s}\right)^{\mathrm{k}}\left[J_{\mathbf{k}}\left(x_{2}\right)-\frac{x_{2} J_{\mathbf{k}}\left(x_{2}\right)}{\mu_{2} k}\right] \frac{D_{\mathrm{k}}}{2}\right\}
\end{gathered}
$$

Since this must be identically true whatever the values of $r_{1} \theta_{1}$, the coefficient of $r^{\mathrm{n}}{ }_{1}$ must vanish for every value of $n=0,1,2,3 \cdots \infty$. If for brevity one writes

$Q_{\mathrm{n}} \equiv-\frac{D_{\mathrm{n}}}{4 \mu_{2}}\left(\frac{a_{2}}{s}\right)^{\mathrm{n}}\left[\mu_{2} n J_{\mathrm{n}}\left(x_{2}\right)-x_{2} J^{\prime}{ }_{n}\left(x_{2}\right)\right]$

and

$\left.P_{\mathrm{n}} \equiv-\frac{C_{\mathrm{n}}}{4 \mu_{1}}\left(\frac{a_{1}}{s}\right)^{\mathrm{n}}\left[\mu_{1} n J_{\mathrm{n}}\left(x_{1}\right)-x_{1} J^{\prime}{ }_{\mathrm{n}}\left(x_{1}\right)\right]\right\}$

this identity gives the set of equations

$$
\begin{aligned}
& \left\{-I_{2} \sum_{k=1}^{\infty} \frac{Q_{\mathrm{k}}}{k}+2 I_{1} P_{\mathrm{o}}\left[\log a_{1}-\frac{\mu_{1} J_{\mathrm{o}}\left(x_{1}\right)}{x_{1} J_{\mathrm{o}}^{\prime}\left(x_{1}\right)}\right]=-2 I_{2} Q_{\mathrm{o}} \log s-\frac{\gamma c_{1}-i p A(\infty)}{2 i p}\right. \\
& I_{2} \sum_{k=1}^{\infty} \frac{n+\mathrm{n}-1}{\mid \underline{n-1 \mid k}} Q_{\mathrm{k}}-\left(\frac{s}{a_{1}}\right)^{2 \mathrm{n}} \frac{\mu_{1} n J_{\mathrm{n}}\left(x_{1}\right)+x_{1} J^{\prime}{ }_{\mathrm{n}}\left(x_{1}\right)}{\mu_{1} n J_{\mathrm{n}}\left(x_{1}\right)-x_{1} J^{\prime}{ }_{\mathrm{n}}\left(x_{1}\right)} \cdot P_{\mathrm{n}} I_{1}=-2 Q_{0} I_{2}
\end{aligned}
$$

The corresponding set

$$
\text { for } n=1,2,3 \cdots \infty
$$

$$
\left\{\begin{array}{c}
-I_{1} \sum_{k=1}^{\infty} \frac{P_{\mathrm{k}}}{k}+I_{2} 2 Q_{\mathrm{o}}\left[\log a_{2}-\frac{\mu_{2} J_{\mathrm{o}}\left(x_{2}\right)}{x_{2} J^{\prime}{ }_{\mathrm{o}}\left(x_{2}\right)}\right]=-2 I_{1} P_{\mathrm{o}} \log s-\frac{\gamma c_{2}-i p A(\infty)}{2 i p} \\
I_{1} \sum_{k=1}^{\infty} \frac{n+k-1}{\underline{n-1}{ }^{n-112)}} P_{\mathrm{k}}-\left(\frac{s}{a_{2}}\right)^{2 \mathrm{n}} \frac{\mu_{2} n J_{\mathrm{n}}\left(x_{2}\right)+x_{2} J^{\prime}{ }_{\mathrm{n}}\left(x_{2}\right)}{\mu_{2} n J_{\mathrm{n}}\left(x_{2}\right)-x_{2} J^{\prime}\left(x_{2}\right)} Q_{\mathrm{n}} I_{2}=-2 P_{\mathrm{o}} I_{1} \\
\text { for } n=1,2,3 \cdots \infty
\end{array}\right.
$$


are obtained from this identity in case 2 where the point $P$ is within the second circle, by permuting the subscripts and constants.

Since $E=\gamma c_{1}-i p A$ in cylinder No. 1

or

$$
I_{1}=i p I_{1} C_{0} 2 \pi \lambda_{1} \int_{0}^{a_{1}} r J_{0}\left(h_{1} r\right) d r=\frac{I_{1} C_{0} x_{1} J^{\prime}{ }_{0}\left(x_{1}\right)}{2 \mu_{1}}=2 I_{1} P_{\circ}
$$

Similarly

$$
2 P_{\mathrm{o}}=+1
$$

Hence

$$
I_{2}=i p I_{2} D_{0} 2 \pi \lambda_{2} \int_{0}^{a_{2}} r J_{0}\left(h_{2} r\right) d r=\frac{I_{2} D_{0} x_{2} J^{\prime}{ }_{0}\left(x_{2}\right)}{2 \mu_{2}}=+I_{2} 2 Q_{0}
$$

$$
2 P_{\mathrm{o}}=2 Q_{\mathrm{o}}=+1
$$

Placing these values of $P_{0}$ and $Q_{0}$ in (111) and (112), and placing $I_{2}=-I_{1}$ gives

$$
\left\{\begin{array}{l}
\sum_{k=1}^{\infty} \frac{\mid n+k-1}{|n-1| \underline{k}} Q_{\mathrm{k}}+\left(\frac{s}{a_{1}}\right)^{2 \mathrm{n}} \frac{\mu_{1} n J_{\mathrm{n}}\left(x_{1}\right)+x_{1} J^{\prime}{ }_{\mathrm{n}}\left(x_{1}\right)}{\mu_{1} n J_{\mathrm{n}}\left(x_{1}\right)-x_{1} J^{\prime}{ }_{\mathrm{n}}\left(x_{1}\right)} P_{\mathrm{n}}=-1 \\
\sum_{k=1}^{\infty} \frac{n+k-1}{|\underline{n-1}| \underline{k}} P_{\mathrm{k}}+\left(\frac{s}{a_{2}}\right)^{2 \mathrm{n}} \frac{\mu_{2} n J_{\mathrm{n}}\left(x_{2}\right)+x_{2} J^{\prime}{ }_{\mathrm{n}}\left(x_{2}\right)}{\mu_{2} n J_{\mathrm{n}}\left(x_{2}\right)-x_{2} J^{\prime}{ }_{\mathrm{n}}\left(x_{2}\right)} Q_{\mathrm{n}}=-1
\end{array}\right\}
$$

for $n=1,2,3 \cdots \cdots \infty$

The two equations for $n=0$ become

$$
\left\{\begin{array}{l}
\gamma c_{1}-i p A(\infty)=2 i p I_{1}\left[\log \frac{s}{a_{1}}+\frac{\mu_{1} J_{o}\left(x_{1}\right)}{x_{1} J^{\prime}{ }_{o}\left(x_{1}\right)}-\sum_{k=1}^{\infty} \frac{Q_{\mathrm{k}}}{k}\right] \\
\gamma c_{2}-i p A(\infty)=-2 i p I_{1}\left[\log \frac{s}{a_{2}}+\frac{\mu_{2} J_{o}\left(x_{2}\right)}{x_{2} J^{\prime}{ }_{0}\left(x_{2}\right)}-\sum_{k=1}^{\infty} \frac{P_{\mathrm{k}}}{k}\right]
\end{array}\right\}
$$

Or, by subtraction

$$
\gamma\left(c_{1}-c_{2}\right)=2 i p I_{1}\left\{\frac{\mu_{1} J^{\prime} \circ\left(x_{1}\right)}{x_{1} J^{\prime}{ }_{0}\left(x_{1}\right)}+\frac{\mu_{2} J_{0}\left(x_{2}\right)}{x_{2} J_{o}\left(x_{2}\right)}+\log \frac{s^{2}}{a_{1} a_{2}}-\sum_{k=1}^{\infty}\left[\frac{P_{\mathrm{k}}+Q_{\mathrm{k}}}{k}\right]\right\}
$$

The second member of this equation will be known when $P_{\mathrm{k}}$ and $Q_{\mathbf{k}}$ for $k=1,2,3 \cdots \infty$ have been found as solutions of the equations (113). The equation of conservation of electricity is

$$
\gamma I_{1}=\left(G_{12}+i p C_{12}\right)\left(c_{1}-c_{2}\right)
$$

where $\frac{1}{G_{12}}$ and $C_{12}$ are, respectively, the insulation resistance and capacity between the two cylinders per unit length. The value of $\gamma$ found by eliminating $\frac{\left(c_{1}-c_{2}\right)}{I_{1}}$ between (115) and (116) is

$$
\begin{aligned}
& \gamma^{2}=\left(G_{12}+i p C_{12}\right) 2 i p\left\{\frac{\mu_{1} J_{0}\left(x_{1}\right)}{x_{1} J^{\prime}{ }_{0}\left(x_{1}\right)}+\frac{\mu_{2} J_{0}\left(x_{2}\right)}{x_{2} J^{\prime}{ }_{0}\left(x_{2}\right)}\right.+\log \frac{s^{2}}{a_{1} a_{2}} \\
&\left.-\sum_{k=1}^{\infty}\left[\frac{P_{\mathrm{k}}+Q_{\mathrm{k}}}{k}\right]\right\}
\end{aligned}
$$


The resistance $R$ and inductance $L$ per unit length of the line are then given by equation (98).

$$
\begin{aligned}
R+i p L & =\frac{\gamma^{2}}{G+i p C} \\
& =2 i p\left\{\frac{\mu_{1} J_{0}\left(x_{1}\right)}{x_{1} J^{\prime}{ }_{0}\left(x_{1}\right)}+\frac{\mu_{2} J_{0}\left(x_{2}\right)}{x_{2} J^{\prime}{ }_{0}\left(x_{2}\right)}+\log \frac{s^{2}}{a_{1} a_{2}}-\sum_{n=1}^{\infty}\left[\frac{P_{\mathrm{n}}+Q_{n}}{n}\right]\right\}
\end{aligned}
$$

The insulation resistance $\frac{1}{G}$ and capacity $C$ between the two wires per unit length are given by

$$
G=\frac{4 \pi \lambda d}{2 \log \frac{1}{b_{1} b_{2}}}, \quad C=\frac{k}{2 c^{2} \log \frac{1}{b_{1} b_{2}}}, \quad c=3(10)^{10}
$$

in electromagnetic cgs units, $\lambda_{\mathrm{d}}$ being the conductivity of the dielectric in these units and $k$ its dielectric constant in cgs electrostatic units. The pure numbers $b_{1}$ and $b_{2}$ are both positive and less than 1 and satisfy the equations

$$
\left.\begin{array}{l}
a_{1} b_{1}\left(s-a_{2} b_{2}\right)=a^{2}{ }_{1} \\
a_{2} b_{2}\left(s-a_{1} b_{1}\right)=a_{2}^{2}
\end{array}\right\}
$$

$s$ being the distance between the centers of the circles. The distance of the image point in No. 1 from its center is $a_{1} b_{1}$ and $a_{2} b_{2}$ is the distance of the image point in No. 2 from the center of the second circle. The explicit formulas for $b_{1}$ and $b_{2}$ are

$$
\left.\begin{array}{l}
b_{1}=\frac{s^{2}+a^{2}{ }_{1}-a_{2}^{2}}{2 s a_{1}}-\sqrt{\left(\frac{s^{2}+a^{2}{ }_{1}-a_{2}{ }_{2}{ }^{2}}{2 s a_{1}}\right)^{2}-1} \\
b_{2}=\frac{s^{2}+a^{2}{ }_{2}-a^{2}{ }_{1}}{2 s a_{2}}-\sqrt{\left(\frac{\left.s^{2}+a^{2}{ }_{2}-a^{2}{ }_{1}\right)^{2}}{2 s a_{2}}-1\right.}
\end{array}\right\}
$$

which are both real, positive, and less than 1 (when the circles are external to each other), but $b_{1}$ and $b_{2}$ both approach the value 1 when the cylinders approach contact, and both approach the value zero when they are widely separated. In case $a_{1}=a_{2}$ then $b_{1}=b_{2}$. If the plus sign were taken in front of the radical, this would give the reciprocal of the value of $b_{1}$ given above, which is the other root of the quadratic equation to determine $b_{1}$, namely,

$$
b_{1}^{2}-\left(\frac{s^{2}+a_{1}^{2}+a_{2}^{2}}{s a_{1}}\right) b_{1}+1=0
$$

The equation for $b_{2}$ is obtained by interchange of subscripts.

In abandoning the strict method of integral equations and assuming that the solution of the problem may be found in the form of a 
series (the Fourier-Bessel expansion) one can not be sure of the existence of a solution of this form, or if a solution is found, there is no assurance that it is unique. Curiously enough, the infinite set of equations (113) which have been obtained for the coefficients $P_{1} P_{2} \ldots Q_{1} Q_{2} \ldots$ admit of two solutions, and that one must be rejected as unphysical which does not satisfy the integral equation. This fact is brought out in the following derivation of asymptotic or high-frequency formulas for the alternating current resistance and inductance.

In case the two cylinders have the same radius, conductivity, and permeability $b_{1}=b_{2}$, and $Q_{\mathrm{n}}=P_{\mathrm{n}}$. This is a problem in current distribution which has been treated by a number of mathematicians, the earliest being Mie. ${ }^{3}$ It has been successfully solved for low frequencies by Curtis, ${ }^{4}$ using a Fourier-Maclaurin expansion. The regular methods of integral equations has also been used in a paper by Maneback, ${ }^{5}$ whose results are also limited in their application. The most thorough treatment, however, is that of Carson, ${ }^{6}$ who has made arithmetical computations from the infinite set of linear equations, and whose results are quite unrestricted as to spacing of the wires or frequency. The problem having circular symmetry has also been solved in a general manner by Carson and Gilbert.?

The remainder of this paper will be devoted to the derivation of high-frequency formulas for the alternating current resistance $R$, inductance $L$, and the attenuation $b$ and phase velocity $v$, which hold for any dimensions or spacing of the two unequal cylinders.

(a) First and Second Approximations at High Frequency. asymptotic Formulas for $R, L$, and $\gamma$ With any Spacing.For high frequency

and

$$
\begin{aligned}
\frac{\mu_{1} n J_{n}\left(x_{1}\right)+x_{1} J^{\prime}{ }_{n}\left(x_{1}\right)}{\mu_{1} n J_{n}\left(x_{1}\right)-x_{1} J^{\prime}{ }_{n}\left(x_{1}\right)} & \approx-1+\frac{2 i \mu_{1}}{x_{1}} n+\text { higher powers of } \frac{1}{x} \\
& \approx-\left\{1+(1-i) n \sqrt{\frac{\mu_{1} R_{1}(0)}{2 p}}\right\} .
\end{aligned}
$$

$$
\frac{\mu_{2} n J_{\mathrm{n}}\left(x_{2}\right)+x_{2} J^{\prime}{ }_{\mathrm{n}}\left(x_{2}\right)}{\mu_{2} n J_{\mathrm{n}}\left(x_{2}\right)-x_{2} J^{\prime}{ }_{\mathrm{n}}\left(x_{2}\right)} \approx-\left\{1+(1-i) n \sqrt{\frac{\mu_{2} R_{2}(0)}{2 p}}\right\}
$$

The equations (113) become

$$
\left.\begin{array}{l}
1+\sum_{k=1}^{\infty} \frac{n+k-1}{n-1} P_{\mathrm{k}}-\left(\frac{s}{a_{2}}\right)^{2 \mathrm{n}} Q_{\mathrm{n}}\left\{1+n(1-i) \sqrt{\frac{\mu_{2} R_{2}(0)}{2 p}}\right\}=0 \\
1+\sum_{k=1}^{\infty} \frac{n+k-1}{n-1} Q_{\mathrm{k}}-\left(\frac{s}{a_{1}}\right)^{2 \mathrm{n}} P_{\mathrm{n}}\left\{1+n(1-i) \sqrt{\frac{\mu_{1} R_{1}(0)}{2_{\mathrm{p}}}}\right\}=0
\end{array}\right\}
$$

I G. Mie, Annalen der Phys., 2, 1900, pp. 201-249.

4 H. L. Curtis, B. S. Sci. Paper No. 374.

B Charles Maneback, Jour. of Math. \& Pbys., Mass. Inst. of Tech., 1, No. 3, A pril, 1922; pp. 123-124.

CJobn R. Carson, Phil .Mag. 41, A pril, 1921.

i J. R. Carson and J. J. Gilbert, Transmission characteristics of the submarine cable, Jour. of the Frank. Inst., December, 1921; pp. 705-735. 
Assume

$$
\left.\begin{array}{l}
P_{\mathrm{n}}=P_{\mathrm{n}}{ }^{(0)}+\frac{1}{\sqrt{2 p}} \dot{P}_{\mathrm{n}}^{(1)} \\
Q_{\mathrm{n}}=Q_{\mathrm{n}}{ }^{(0)}+\frac{1}{\sqrt{2 p}} Q_{\mathrm{n}}^{(1)}
\end{array}\right\}
$$

Substituting these forms in the above set of equations and equating like powers of $\frac{1}{\sqrt{2 p}}$ gives

and

$$
\left.\begin{array}{l}
1+\sum_{k=1}^{\infty} \frac{\frac{n+k-1}{n-1} P_{\mathrm{k}}{ }^{(0)}=\left(\frac{s}{a_{2}}\right)^{2 \mathrm{n}} Q_{\mathrm{n}}{ }^{(0)}}{1+\sum_{k=1}^{\infty} \frac{n+k-1}{n-1} Q_{\mathbf{k}}{ }^{(0)}=\left(\frac{s}{a_{1}}\right)^{2 \mathrm{n}} P_{\mathbf{n}}{ }^{(0)}}
\end{array}\right\} n=1,2,3 \ldots \ldots \infty
$$

$$
\begin{aligned}
& \sum_{k=1}^{\infty} \frac{n+k-1}{\underline{n-1} \mid k} P_{\mathbf{k}}^{(1)}-\left(\frac{s}{a_{2}}\right)^{2 \mathrm{n}} Q_{\mathbf{n}}^{(1)}=n\left(\frac{s}{a_{2}}\right)^{2 \mathrm{n}} Q_{\mathrm{n}}{ }^{(0)}(1-i) \sqrt{\mu_{2} R_{2}(0)} \\
& \left.\sum_{k=1}^{\infty} \frac{n+k-1}{\underline{n-1} \mid \underline{k}} Q_{\mathrm{k}}^{(1)}-\left(\frac{s}{a_{1}}\right)^{2 \mathrm{n}} \quad P_{\mathrm{n}}^{(1)}=n\left(\frac{s}{a_{1}}\right)^{2 \mathrm{n}} P_{\mathrm{n}}^{(0)}(1-i) \sqrt{\mu_{1} R_{1}(0)}\right\}
\end{aligned}
$$

The equations (125) can be solved exactly, and the values of $P_{n}{ }^{(0)}$ and $Q_{\mathrm{n}}{ }^{(0)}$ then substituted in (126) and the resulting equations solved exactly as follows:

Since

$$
\text { Exact solution of the first system of equations (125) }
$$

$$
1+\sum_{k=1}^{\infty} \frac{\mid n+k-1}{\underline{n-1} \mid \underline{k}} z^{k}=\frac{1}{(1-z)^{n}} \text { if }|z|<1
$$

the equations (125) for $P_{n}{ }^{(0)}$ and $Q_{n}{ }^{(0)}$ suggest the forms

$$
P_{\mathrm{n}}{ }^{(0)}=z^{\mathrm{n}}{ }_{1} \quad Q_{\mathrm{n}}{ }^{(0)}=z^{\mathrm{n}}{ }_{2} \quad \text { where } \quad \begin{aligned}
& \left|z_{1}\right|<1 \\
& z_{2} \mid<1
\end{aligned}
$$

and where $z_{1}$ and $z_{2}$ are to be determined. Substituting in the equations (125) gives

$$
\begin{aligned}
& 1+\sum_{k=1}^{\infty} \frac{n+k-1}{\underline{n-1} \mid \underline{k}} z^{\mathbf{k}}=\frac{1}{\left(1-z_{1}\right)^{\mathrm{n}}}=\left(\frac{s}{a_{2}}\right)^{2 \mathrm{n}} z^{{ }^{\mathrm{n}}}{ }_{2} \\
& \left.1+\sum_{k=1}^{\infty} \frac{n+k-1}{n-1[k} z^{\mathrm{k}}=\frac{1}{\left(1-z_{2}\right)^{\mathrm{n}}}=\left(\frac{s}{a_{1}}\right)^{2 \mathrm{n}} z^{\mathrm{n}}{ }_{1}\right\} n=1,2,3 \cdots \infty
\end{aligned}
$$


which shows that the $2 \infty$ equations will all be satisfied if $z_{1}$ and $z_{2}$ can be found each numerically less than 1 , satisfying the two equations

$$
\left.\begin{array}{l}
\left(\frac{s z_{1}}{a_{1}}\right)\left[s-a_{2}\left(\frac{s z_{2}}{a_{2}}\right)\right]=a_{1} \\
\left(\frac{s z_{2}}{a_{2}}\right)\left[s-a_{1}\left(\frac{s z_{1}}{a_{1}}\right)\right]=a_{2}
\end{array}\right\}
$$

By reference to the equations (120) which determine $b_{1}$ and $b_{2}$ it is evident that

$$
z_{1}=\frac{a_{1} b_{1}}{s} \quad \text { and } \quad z_{2}=\frac{a_{2} b_{2}}{s}
$$

where $b_{1}$ and $b_{2}$ are roots of the corresponding quadratic equations. In order that $z_{1}$ and $z_{2}$ shall be less than $1, b_{1}$ and $b_{2}$ must both be less than 1, or both greater than 1 . Since the product of the two roots of each equation is 1 , it follows that there are two solutions for the above system of equations (125) given by

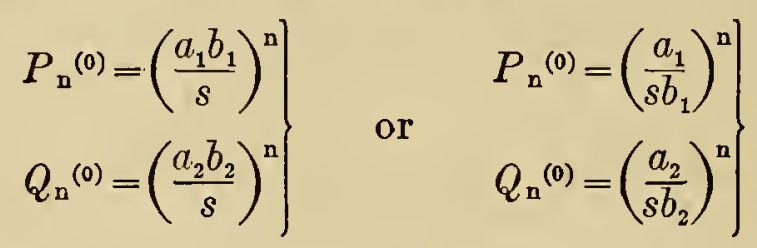

It will appear presently that the set corresponding to $b_{1}$ and $b_{2}$ both less than unity leads to positive expressions for the resistance and inductance, while the other set lead to the same numerical values, but a negative sign. Using these values of $P_{n}{ }^{(0)}$ and $Q_{n}{ }^{(0)}$ in (126) leads to the following equations for the $P_{n}{ }^{(1)}$ and $Q_{n}{ }^{(1)}$

Exact solution of the second system of equations (126)

To find $P_{\mathbf{n}}{ }^{(1)}$ and $Q_{n}{ }^{(1)}$ as solutions of

$$
\left.\begin{array}{l}
\sum_{k=1}^{\infty} \frac{\mid n+k-1}{\mid \frac{n}{n}} P_{\mathrm{k}}^{(1)}-\left(\frac{s}{a_{2}}\right)^{2 \mathrm{n}} \frac{Q_{\mathrm{n}}^{(1)}}{n}=\left(\frac{s b_{2}}{a_{2}}\right)^{\mathrm{n}}(1-i) \sqrt{\mu_{2} R_{2}(0)} \\
\sum_{k=1}^{\infty} \frac{\mid n+k-1}{\mid \frac{n}{\mid k}} Q_{\mathrm{k}}^{(1)}-\left(\frac{s}{a_{1}}\right)^{2 \mathrm{n}} \frac{P_{\mathrm{n}}^{(1)}}{n}=\left(\frac{s b_{1}}{a_{1}}\right)^{\mathrm{n}}(1-i) \sqrt{\mu_{1} R_{1}(0)}
\end{array}\right\}
$$

Assume

$$
P_{\mathrm{n}}{ }^{(1)}=\alpha n z^{\mathrm{n}}{ }_{1} \quad \text { and } \quad Q_{\mathrm{n}}{ }^{(1)}=\beta n z^{\mathrm{n}}{ }_{2} \text { where } \begin{aligned}
& \left|z_{1}\right|<1 \\
& \left|z_{2}\right|<1
\end{aligned}
$$

and substitute in above equations (129). This gives

$$
\begin{aligned}
& \alpha z_{1} \sum_{k=1}^{\infty} \frac{n+k-1}{\underline{n} \mid \underline{k}-1} z_{1}{ }^{\mathrm{k}-1}-\beta\left(\frac{s}{a_{2}}\right)^{2 \mathrm{n}} z^{\mathrm{n}}{ }_{2}=\left(\frac{s b_{2}}{a_{2}}\right)^{\mathrm{n}}(1-i) \sqrt{\mu_{2} R_{2}(0)} \\
& \beta z_{2} \sum_{k=1}^{\infty} \frac{n+k-1}{\underline{n} \mid k-1} z_{1}{ }^{k-1}-\alpha\left(\frac{s}{a_{1}}\right)^{2 \mathrm{n}} z^{\mathrm{n}}{ }_{1}=\left(\frac{s b_{1}}{a_{1}}\right)^{\mathrm{n}}(1-i) \sqrt{\mu_{1} R_{1}(0)}
\end{aligned}
$$


Or, since by the binominal theorem

$$
\begin{gathered}
\sum_{k=1}^{\infty} \frac{\mid \frac{n+k-1}{n \underline{n-1}} z_{1}{ }^{\mathrm{k}-1}=\frac{1}{\left(1-z_{1}\right)^{\mathrm{n}+1}}}{\frac{\alpha z_{1}}{1-z_{1}} \frac{1}{\left(1-z_{1}\right)^{\mathrm{n}}}-\beta\left(\frac{s^{2} z_{2}}{a^{2}{ }_{2}}\right)^{\mathrm{n}}=\left(\frac{s b_{2}}{a_{2}}\right)^{\mathrm{n}}(1-i) \sqrt{\mu_{2} R_{2}(0)}} \\
\frac{\beta z_{2}}{1-z_{2}} \frac{1}{\left(1-z_{2}\right)^{\mathrm{n}}}-\alpha\left(\frac{s^{2} z_{1}}{a^{2}}\right)^{\mathrm{n}}=\left(\frac{s b_{1}}{a_{1}}\right)^{\mathrm{n}}(1-i) \sqrt{\mu_{1} R_{1}(0)}
\end{gathered}
$$

If these equations are to hold for every positive integral value of $n$, this requires that $\alpha$ and $\beta$ have certain values, and that

$$
\left.\begin{array}{l}
z_{2}=\frac{a_{2} b_{2}}{s}=\frac{\left(\frac{a_{2}}{s}\right)^{2}}{1-z_{1}} \\
z_{1}=\frac{a_{1} b_{1}}{s}=\frac{\left(\frac{a_{1}}{s}\right)^{2}}{1-z_{2}}
\end{array}\right\}
$$

where $z_{1}, z_{2}, b_{1}$ and $b_{2}$ must be the same as in the preceding case. Since

$$
\frac{z_{1}}{1-z_{1}}=\frac{a_{1} b_{1} b_{2}}{a_{2}} \text { and } \frac{z_{2}}{1-z_{2}}=\frac{a_{2} b_{1} b_{2}}{a_{1}}
$$

the constants $\alpha$ and $\beta$ must be so chosen as to satisfy

$$
\left.\begin{array}{rl}
-a_{1} \alpha+a_{2} b_{1} b_{2} \beta & =(1-i) a_{1} \sqrt{\mu_{1} R_{1}(0)} \\
a_{1} b_{1} b_{2} \alpha-a_{2} \beta & =(1-i) a_{2} \sqrt{\mu_{2} R_{2}(0)}
\end{array}\right\}
$$

which being solved, give

$$
\left.\begin{array}{l}
a_{1} \alpha=-\frac{(1-i)}{\left(1-b^{2} b^{2}{ }_{2}\right)}\left[a_{1} \sqrt{\mu_{1} R_{1}(0)}+b_{1} b_{2} a_{2} \sqrt{\mu_{2} R_{2}(0)}\right] \\
a_{2} \beta=-\frac{(1-i)}{\left(1-b^{2} b_{1}^{2}{ }_{2}\right)}\left[b_{1} b_{2} a_{1} \sqrt{\mu_{1} R_{1}(0)}+a_{2} \sqrt{\mu_{2} R_{2}(0)}\right]
\end{array}\right\}
$$

Introducing the results of the first and second stages of the approximation, namely,

$$
\left.\begin{array}{l}
P_{\mathrm{n}}=P_{\mathrm{n}}{ }^{(0)}+\frac{1}{\sqrt{2 p}} P_{\mathrm{n}}^{(1)}=\left(\frac{a_{1} b_{1}}{s}\right)^{\mathrm{n}}\left(1+\frac{n \alpha}{\sqrt{2 p}}\right) \\
Q_{\mathrm{n}}=Q_{\mathrm{n}}{ }^{(0)}+\frac{1}{\sqrt{2 p}} Q_{\mathrm{n}}(1)=\left(\frac{a_{2} b_{2}}{s}\right)^{\mathrm{n}}\left(1+\frac{n \beta}{\sqrt{2 p}}\right)
\end{array}\right\}
$$


into the equation (118) for $R$ and $L$ leads to two infinite series which are summable. For

$$
\begin{aligned}
\log \frac{s}{a_{1}} & -\sum_{n=1}^{\infty} \frac{P_{n}}{n}=\log \frac{s}{a_{1}}-\sum_{n=1}^{\infty} \frac{1}{n}\left(\frac{a_{1} b_{1}}{s}\right)-\frac{\alpha}{\sqrt{2 p}} \sum_{n=1}^{\infty}\left(\frac{a_{1} b_{1}}{s}\right)^{n} \\
& =\log \frac{s}{a_{1}}+\log \left(1-\frac{a_{1} b_{2}}{s}\right)-\frac{\alpha}{\sqrt{2 p}}\left(\frac{1}{1-\frac{a_{1} b_{1}}{s}}-1\right) \\
& =\log \frac{a_{2}}{a_{1} b_{2}}-\frac{\alpha}{\sqrt{2 p}} \frac{a_{1} b_{1} b_{2}}{a_{2}}
\end{aligned}
$$

Similarly

$$
\log \frac{s}{a_{2}}-\sum_{n=1}^{\infty} \frac{Q_{\mathrm{n}}}{n}=\log \frac{a_{1}}{a_{2} b_{1}}-\frac{\beta}{\sqrt{2 p}} \frac{a_{2} b_{1} b_{2}}{a_{1}}
$$

and

$$
\log \frac{s^{2}}{a_{1} a_{2}}-\sum_{n=1}^{\infty} \frac{P_{\mathrm{n}}+Q_{n}}{n}=\log \frac{1}{b_{1} b_{2}}-\frac{b_{1} b_{2}}{\sqrt{2 p}}\left(\frac{\alpha a_{1}}{a_{2}}+\frac{\beta a_{2}}{a_{1}}\right)
$$

Substituting in (118) gives

$$
\begin{aligned}
& R+i p L=2 i p\left\{\frac{\mu_{1} J_{o}\left(x_{1}\right)}{x_{1} J^{\prime}{ }_{o}\left(x_{1}\right)}+\frac{\mu_{2} J_{0}\left(x_{2}\right)}{x_{2} J^{\prime}{ }_{o}\left(x_{2}\right)}+\log \frac{1}{b_{1} b_{2}}\right. \\
& +\frac{(1-i) b_{1} b_{2}}{\left(1-b^{2} b^{2}{ }_{2}\right) \sqrt{2 p}}\left[\frac{a_{1}}{a_{2}} \sqrt{\mu_{1} R_{1}(0)}+\frac{a_{2}}{a_{1}} \sqrt{\mu_{2} R_{2}(0)}\right. \\
& \left.\left.+b_{1} b_{2}\left(\sqrt{\mu_{1} R_{1}(0)}+\sqrt{\mu_{2} R_{2}(0)}\right)\right]\right\}
\end{aligned}
$$

But to the same degree of approximation as used in obtaining $P_{\mathrm{n}}$ and $Q_{n}$; that is, neglecting $\frac{1}{p}$ compared to $\frac{1}{\sqrt{p}}$

and

$$
\frac{\mu_{1} J_{o}\left(x_{1}\right)}{x_{1} J^{\prime}{ }_{0}\left(x_{1}\right)}=-\frac{i \mu_{1}}{x_{1}}=\frac{(1-i)}{2} \sqrt{\frac{\mu_{1} R_{1}(0)}{2 p}}
$$

$$
\frac{\mu_{2} J_{0}\left(x_{2}\right)}{x_{2} J^{\prime}\left(x_{2}\right)}=\frac{(1-i)}{2} \sqrt{\frac{\mu_{2} R_{2}(0)}{2 p}}
$$

Using these expressions in the preceding equation (134) gives, upon equating reals and imaginaries,

$$
\begin{gathered}
R=\frac{1}{2\left(1-b^{2}{ }_{1} b_{2}{ }_{2}\right)}\left[\left(1+2 b_{1} b_{2} \frac{a_{1}}{a_{2}}+b^{2}{ }_{1} b^{2}\right) \sqrt{2 p \mu_{1} R_{1}(0)}+\left(1+2 b_{1} b_{2} \frac{a_{2}}{a_{1}}\right.\right. \\
\left.\left.+b^{2}{ }_{1} b_{2}{ }_{2}\right) \sqrt{2 p \mu_{2} R_{2}(0)}\right]=\frac{1+b_{1} b_{2}}{1-b_{1} b_{2}}\left(\frac{1}{a_{1}}+\frac{1}{a_{2}}\right) \sqrt{\frac{\mu f}{\lambda}} \text { if }\left\{\begin{array}{l}
\mu_{1}=\mu_{2}=\mu \\
\lambda_{1}=\lambda_{2}=\lambda
\end{array}\right. \\
L=2 \log \frac{1}{b_{1} b_{2}}+\frac{R}{p}=\frac{c^{2} C}{k}+\frac{R}{p}
\end{gathered}
$$


The attenuation $b$ and phase velocity $V$ are given by

or

$$
\left.\begin{array}{l}
\gamma \equiv b+i \frac{p}{\bar{V}}=\sqrt{(R+i p L))(G+i p C} \\
2 b^{2}=\sqrt{\left(R^{2}+p^{2} L^{2}\right)\left(G^{2}+p^{2} C^{2}\right)}+\left(R G-p^{2} L C\right) \\
2 \frac{p^{2}}{V^{2}}=\sqrt{\left(R^{2}+p^{2} L^{2}\right)\left(G^{2}+p^{2} C^{2}\right)}-\left(R G-p^{2} L C\right)
\end{array}\right\}
$$

The ratio $\frac{G}{p C}$; that is, $\frac{4 \pi \lambda_{\mathrm{d}} c^{2}}{p k}$ is generally negligible, so that (137) reduces to

$$
\nabla=\frac{1}{\sqrt{L C}} \quad b=\frac{R C V}{2}
$$

The positive quantity $b_{1} b_{2}$ which appears in these formulas may be found as the root of the quadratic equation

$$
\left(b_{1} b_{2}\right)^{2}-\left(\frac{s^{2}-a_{1}^{2}-a^{2}{ }_{2}}{a_{1} a_{2}}\right) b_{1} b_{2}+1=0
$$

which is less than 1 . The other root, being the reciprocal of this, will be greater than 1 , and if used in these formulas would give the same numerical value of $R$ and $L$, but both negative in sign.

Special Case.-The circles have equal radii, $b_{2}=b_{1}$

$$
\begin{gathered}
R=\left\{\frac{\sqrt{\mu_{1} R_{1}(0)}+\sqrt{\mu_{2} R_{2}(0)}}{2}\right\} \sqrt{\frac{2 p}{1-\left(\frac{2 a}{s}\right)^{2}}} \\
L=4 \log \frac{1}{b_{1}}+\frac{R}{p}
\end{gathered}
$$

where $b_{1}$ is that root of

$$
b^{2}{ }_{1}-\left(\frac{s}{a_{1}}\right) b_{1}+1=0 \text { which is less than } 1
$$

or

$$
b_{1}=\frac{s}{2 a_{1}}-\sqrt{\left(\frac{s}{2 a_{1}}\right)^{2}-1}
$$

The asymptotic expressions for alternating current resistance and inductance of two cylinders here given are believed to be new. They have proven useful in the radio section of this bureau for investigating the precision of measurements of short waves on wires, corresponding to a frequency of $(10)^{7}$ cycles per second. ${ }^{8}$

(b) Special Case. Circular Cylinder Parallel to Semiinfinite Plane of Finite Conduotivity.- If we plaoe $s=d+a_{2}$ and holding $d$ fixed let $a_{2}$ become infinite, this approaches the case

A. Hund, B. S. Sci. Paper No. 491 
of a cylinder of radius $a_{1}$, whose center is a distance $d$ from the plane boundary of a semi-infinite solid of finite conductivity. This is the case of a single cylinder a height $d$ above the earth, the latter being the return conductor. In this case

$$
b_{1} b_{2}=\frac{d-\sqrt{d^{2}-a_{1}^{2}}}{a_{1}}
$$

Formulas (135) to (138) then simplify, and they show the manner in which the conductivity of the ground $\lambda_{2}$ affects the propagation along a horizontal antenna at radio frequencies. In case $\mu_{1}=\mu_{2}=1$, formula (135) gives for the resistance per unit length of the circuit made of a horizontal antenna and the ground, where $d$ is large compared to $a_{1}$

$$
R=\left(\frac{1}{a_{1} \sqrt{\lambda_{1}}}+\frac{1}{d \sqrt{\lambda_{2}}}\right) \sqrt{f}
$$

where $f$ is the frequency, $a_{1}$ and $\lambda_{1}$ the radius and conductivity of the antenna, and $d$ and $\lambda_{2}$ its height above ground and the conductivity of the latter, respectively.

As a numerical example of the use of (135) to (139) consider a two-conductor cable of copper wires of radii $a_{1}=0.3 \mathrm{~cm}, a_{2}=0.5 \mathrm{~cm}$, with a distance $s=1 \mathrm{~cm}$ between centers and surrounded by a large amount of insulating material whose dielectric constant is $k=4$ electrostatic cgs units. Suppose that the electrical conductivity of this material $\lambda_{d}$ is less than about $10^{-20}$ electromagnetic cgs units, and the frequency $f$ is $10^{5}$ cycles per second. For this value of the frequency (or higher values) the insulation leakage $G$ has no appreciable effect, for

$$
\frac{G}{p C}=\frac{4 \pi c^{2} \lambda_{\mathrm{d}}}{2 \pi f k}=\frac{4 \pi \times 3^{2} \times 10^{20} \times 10^{-20}}{2 \pi \times 4 \times 10^{5}}=4 \times 10^{-5}
$$

which is negligible compared to unity and this indicates the error involved by neglecting $G$.

The direct-current resistance of the wires per unit length in electromagnetic cgs units is (since $\lambda=0.0006$ for copper)

$$
\begin{aligned}
& R_{1}(0)=\frac{1}{\pi a^{2}{ }_{1} \lambda}=\frac{1}{\pi(.3)^{2} .0006}=5.9 \times(10)^{3} \\
& R_{2}(0)=\frac{1}{\pi a_{2}^{2} \lambda}=\frac{1}{\pi(.5)^{2} .0006}=2.1 \times(10)^{3}
\end{aligned}
$$

The direct current resistence of the line per unit length is

$$
\begin{aligned}
R_{1}(0)+R_{2}(0) & =8 \times 10^{3} \\
\sqrt{2 p R_{1}(0)} & =\frac{2}{a_{1}} \sqrt{\frac{f}{\lambda}}=\frac{2}{a_{1}} \times 1.3 \times 10^{4} \\
\sqrt{2 p R_{2}(0)} & =\frac{2}{a_{2}} \sqrt{\frac{f}{\lambda}}=\frac{2}{a_{2}} \times 1.3 \times 10^{4}
\end{aligned}
$$


The equation (135) for the alternating current resistance of the line per unit length is

$$
R=\frac{1+b_{1} b_{2}}{1-b_{1} b_{2}}\left(\frac{1}{.3}+\frac{1}{.5}\right) 1.3 \times 10^{4}=6.93 \frac{1+b_{1} b_{2}}{1-b_{1} b_{2}} \times 10^{4}
$$

(electromagnetic cgs units)

The numerical value of $b^{1} b^{2}$ is found as that root of the quadratic equation (139)

$$
\left(b_{1} b_{2}\right)^{2}-\frac{1-.3^{2}-.5^{2}}{.3 \times .5}\left(b_{1} b_{2}\right)+1=0
$$

which is less than 1 . The two roots are $b^{2} b^{1}=.24$ or 4.16 . The former value must be used, and it gives

$$
R=6.93\left(\frac{1.24}{.76}\right) \times 10^{4}=113 \times 10^{3}
$$

as the alternating current resistance of the line per unit length in electromagnetic cgs units. This is 14 times the direct current resistance. After $R$ has been computed, the alternating current inductances $L$ of the line per unit length may be computed by (136).

$$
L=2 \log _{e} \frac{1}{.24}+\frac{113 \times 10^{3}}{2 \pi 10^{5}}=2.85+.18=3.03
$$

(electromagnetic egs units per $\mathrm{cm}$ )

The capacity of the line per unit length is given by (119)

$$
C=\frac{4}{3^{2} \times 10^{20} \times 2.85}=1.56 \times 10^{-21}
$$

(electromagnetic cgs units)

The phase velocity $V$ is next found by means of (138)

$$
V=\frac{1}{\sqrt{L C}}=\frac{1}{\sqrt{3.03 \times 1.56 \times 10^{-21}}}=1.45 \times 10^{10}
$$

which is about half the velocity of light in space. However, the velocity of light in the medium which is assumed to have a dielectric capacity $k=4$ is

$$
V_{\mathrm{o}}=\frac{\mathrm{c}}{\sqrt{k}}=\frac{3}{\sqrt{4}} \times 10^{10}=1.50 \times 10^{10}
$$

which shows that $V$ is very nearly $V_{\mathrm{o}}$. In this problem the dielectric whose specific inductive capacity is 4 electrostatic cgs units is assumed to fill the space external to the wires. 
Finally the attenuation constant $b$ is found by (137)

$$
b=\frac{R C V}{2}=\frac{113 \times 10^{3} \times 1.56 \times 10^{-21} \times 1.45 \times 10^{10}}{2}=1.28 \times 10^{-0}
$$

The current $I$ is of the form

$$
I=I_{0} e^{-\mathrm{bz}} \cos 2 \pi f\left(t-\frac{z}{V}\right)
$$

and the wave length is

$$
\frac{V}{f}=\frac{1.45 \times 10^{10}}{10^{5}}=1,450 \text { meters }
$$

\section{SUMMARY}

In a single type of wave the field components are proportional to $e^{\text {ipt }-\gamma z}$ where the propagation constant $\gamma$ is $b+\frac{i p}{V}$, the real constants $b$ and $V$ being the attenuation and phase velocity, respectively. They are functions of the frequency. There are $N-1$ possible values of $\gamma^{2}$ and $2 N-2$ values of $\gamma$ or types of waves for a system of $N$ cylindrical conductors. The electromagnetic field is, in general, the superposition of the fields corresponding to each type and these are derivable from a complex scalar potential $\phi$, and a vector potential $A$. On account of the vast difference in the order of magnitude of the electrical conductivities of a conductor and of a dielectric, certain approximations can be made in general which are valid from the lowest to the highest or radio frequencies. These lead to the conclusion that the $x-$ and $y$ - components of the vector potential are negligible.

Beginning with the differential equations and boundary conditions which $\dot{\phi}$ and $A$ must satisfy, the existence of a solution $\phi$ has been proven, and an integral representation of it obtained and its properties studied by constructing a symmetrical auxiliary function of two points $G(x y \xi \eta)$, which may be regarded as a generalization of Green's function. By its means the unknown charges on the boundaries between different dielectrics are eliminated from consideration and $\phi$ is determined at all points in terms of its values on the conducting sections. Certain constants $G_{\mathrm{nk}}$ and $C_{\mathrm{nk}}$ are derived which in general are complex functions of the frequency, and are coefficients of leakage and capacity, respectively. The conservation of electricity for each conductor takes the form, for $n=1,2,3 \cdots N$.

and

$$
\gamma I_{\mathrm{n}}=\sum_{k=1}^{N}\left(G_{\mathrm{nk}}+i p C_{\mathrm{nk}}\right) c_{\mathrm{k}} \text { where }\left\{\begin{array}{l}
G_{\mathrm{nk}}=C_{\mathrm{kn}} \\
C_{\mathrm{nk}}=C_{\mathrm{kn}}
\end{array}\right.
$$

$$
\sum_{k=1}^{N} G_{\mathrm{n} \mathbf{k}}=\sum_{k=1}^{N} C_{\mathrm{n} \mathbf{k}}=0
$$


In a similar manner an integral equation has been obtained for the vector potential $A$ by devising a symmetrical function of two points $M(x y \xi \eta)$ which may be called a magnetic flux function. The existence and uniqueness of a solution of this equation has been proven and methods of solving it discussed. The form and properties of the solution are studied. Its value at all points in conductors may be found without reference to the dielectric. The function $M$ thus eliminates from consideration the dielectric as well as the surface distribution of magnetism existing at the boundaries between different magnetic materials. The real coefficients of resistance and inductance $R_{\mathrm{nk}}$ and $L_{\mathrm{nk}}$ are derived which have the symmetrical property $R_{\mathrm{nk}}=R_{\mathrm{kn}}, L_{\mathrm{nk}}=L_{\mathrm{kn}}$, and in terms of these the definition of current leads to the form

$$
\gamma c_{\mathrm{n}}-i p A(\infty)=\sum_{k=1}^{N}\left(R_{\mathrm{nk}}+i p L_{\mathrm{nk}}\right) I_{\mathrm{k}} \text { for } n=1,2,3 \ldots N
$$

These equations are rendered compatible with those for the conservation of electricity by choosing $\gamma$ a root of a certain determinant which leads to an algebraic equation of degree $N-1$ in $\gamma^{2}$. It is shown that there is but one arbitrary constant for each type of propagation, and all these may be determined when the terminal apparatus at both ends of the line are given. Thus, the attenuation, phase velocity, and reactance of the line may be found. Formulas are also developed for the heating in conductors, and in dielectric, and for the mean electrical and electrokinetic energy of the system in terms of the coefficients above mentioned. Application is made to a pair of circular cylindrical conductors of unequal radii, conductivity, and permeability, and high-frequency formulas derived for the resistance $R$ and inductance $L$ of the line per unit length, as well as for the attenuation $b$ and phase velocity $V$. These are believed to be new.

\section{INDEX OF PRINCIPAL SYMBOLS}

$E\left(E_{\mathrm{x}}, E_{\mathrm{y}}, E_{\mathrm{z}}\right)=$ electric vector.

$H\left(H_{\mathrm{x}}, H_{\mathrm{y}}, H_{\mathrm{z}}\right)=$ magnetic vector.

$B\left(B_{\mathrm{x}}, B_{\mathrm{y}}, B_{\mathrm{z}}\right)=$ magnetic induction.

$f=$ frequency.

$p=2 \pi f$.

$p=2 \pi f$.
$\gamma=$ propagation constant $=b+\frac{i p}{V} \quad i=\sqrt{-1}$

$b=$ attenuation constant.

$V=$ phase velocity.

$c=3(10)^{10}=$ the ratio of the two cgs electrical units.

$\mu=$ magnetic permeability.

$k=$ dielectric constant.

$\lambda=$ electrical conductivity. 
All constants and vectors measured in cgs electromagnetic units except $k$ which is measured in electrostatic cgs units so that

$k=1$ to 5 for most dielectrics.

$\mu=1$ for nonmagnetic metals.

$\phi=$ complex scalar potential.

$A\left(A_{\mathrm{x}}, A_{\mathrm{y}}, A_{z}\right)=$ complex vector potential.

Note.-Since $A_{\mathrm{z}}$ is the principal component considered $A_{\mathrm{z}}$ is written as $A$ where this can be done without misunderstanding. Similarly, $E$ is used for $E_{z}$ where the meaning is plain.

$c_{1}, c_{2}, \cdots c_{\mathrm{r}}=$ complex constant values of $\phi$ upon conducting sections Nos. $1,2,3, \cdots N$.

$a_{\mathrm{j}} \equiv \lambda_{\mathrm{j}}+\frac{i p k_{\mathrm{j}}}{4 \pi c^{2}}=$ complex conductivity of $j^{\text {th }}$ material.

$d s_{\mathrm{j}}=$ element of arc of natural boundary of section $S_{\mathrm{j}}$ of homogeneous material (conductor or dielectric).

$n_{\mathrm{s}}=$ normal to this boundary curve, see Figure 1.

$Q_{\mathrm{n}}=$ complex free charge per unit length upon $n^{\text {th }}$ conducting group.

$G_{\mathrm{n}}=$ leakage current (complex) from $n^{\text {th }}$ conducting group per unit length.

$G_{\mathrm{nk}}$ and $C_{\mathrm{nk}}=$ real coefficients of leakage and capacity, respectively, defined by (26).

$I_{\mathrm{n}}=z$-component of total conduction current through the section $S_{\mathrm{n}}$ of the $n^{\text {th }}$ conducting group.

$G(x y \xi \eta)=$ a generalized Green's function $\}$ defined where intro$M(x y \xi \eta)=$ a magnetic flux function $\}$ duced.

$R_{\mathrm{nk}}$ and $L_{\mathrm{nk}}=$ coefficients of resistance and inductance; defined by (81).

$R$ and $L=$ the alternating current resistance and inductance per unit length, respectively, for a simple return circuit of two conductors. (Equations (135) and (136).)

Washington, January 15, 1925. 